\title{
TWO-DIMENSIONAL MODELS OF TYPE THEORY
}

\author{
RICHARD GARNER
}

\begin{abstract}
We describe a non-extensional variant of Martin-Löf type theory which we call two-dimensional type theory, and equip it with a sound and complete semantics valued in 2-categories.
\end{abstract}

\section{INTRODUCTION}

This is the second in a series of papers detailing the author's investigations into the intensional type theory of Martin-Löf, as described in [25]. The first of these papers, [10], investigated syntactic issues relating to its dependent product types. The present paper is a contribution to its categorical semantics.

In [27] it was proposed that the correct categorical models for extensional Martin-Löf type theory should be locally cartesian closed categories: these being categories $\mathcal{C}$ with finite limits in which each of the functors $f^{*}: \mathcal{C} / X \rightarrow \mathcal{C} / Y$ induced by pulling back along a morphism $f: Y \rightarrow X$ has a right adjoint. The idea is to think of each object $X$ of a locally cartesian closed category $\mathcal{C}$ as a closed type, each morphism as a term, and each object of the slice category $\mathcal{C} / X$ as a type dependent upon $X$. Now substitution of terms in types may be interpreted by pullback between the slices of $\mathcal{C}$; dependent sum and product types by left and right adjoints to pullback; and the equality type on $X$ by the diagonal morphism $\Delta: X \rightarrow X \times X$ in $\mathcal{C} / X \times X$. It was later pointed out in [16] that this picture, whilst very appealing, is not wholly accurate, since in the syntax, the operation which to each morphism of types $f: Y \rightarrow X$ assigns the corresponding substitution operation $\operatorname{Type}(X) \rightarrow \operatorname{Type}(Y)$ is strictly functorial in $f$; whilst in the semantics, the corresponding assignation $(f: Y \rightarrow X) \mapsto\left(f^{*}: \mathcal{C} / X \rightarrow \mathcal{C} / Y\right)$ is rarely so. Thus this notion of model is not sound for the syntax, and we are forced to refine it slightly: essentially by equipping our locally cartesian closed category with a split fibration $\mathcal{T} \rightarrow \mathcal{C}$ equivalent to its codomain fibration $\mathcal{C}^{2} \rightarrow \mathcal{C}$. Types over $X$ are now interpreted as objects of the fibre category $\mathcal{T}(X)$; and since $\mathcal{T} \rightarrow \mathcal{C}$ is a split fibration, the interpretation is sound for substitution.

The question of how the above should generalise from extensional to intensional MartinLöf type theory is a delicate one. It is possible to paraphrase the syntax of intensional type theory in categorical language and so arrive at a notion of model - as done in [6] or [15], for example - but then we lose sight of a key aspect of the extensional semantics, namely that dependent sums and products may be characterised universally, as left and right adjoints to substitution. To obtain a similar result for the intensional theory requires a more refined sort of semantics. More specifically, we are thinking of a semantics valued in higher-dimensional categories, motivated by work such as $[2,9,17]$ which identifies in

Date: 11 September 2008; Revised 21 January 2009.

Supported by a Research Fellowship of St John's College, Cambridge and a Marie Curie IntraEuropean Fellowship, Project No. 040802. 
intensional type theory certain higher-dimensional features. The idea is that, in such a semantics, we should be able to characterise dependent sums and products universally in terms of weak, higher-dimensional adjoints to substitution.

Eventually we expect to be able to construct a sound and complete semantics for intensional type theory valued in weak $\omega$-categories. At the moment the theory of weak $\omega$-categories is insufficiently well-developed for us to describe this semantics; yet we can at least take steps towards it, by describing semantics valued in simpler kinds of higher-dimensional category. In this paper, we describe such a semantics valued in 2categories; which, as well as objects representing types, and morphisms $f: X \rightarrow Y$ representing terms, has 2-cells $\alpha: f \Rightarrow g$ representing witnesses for the propositional equality of terms $f$ and $g$. Intuitively, the 2-categorical models we consider provide a notion of two-dimensional locally cartesian closed category; though bearing in mind the above concerns regarding the functoriality of substitution, it is in fact a "split" notion of two-dimensional model which we will describe here. Relating this to a notion of twodimensional local cartesian closed category will require a 2-categorical coherence result along the lines of [16], and we defer this to a subsequent paper.

Our 2-categorical semantics is sound and complete neither for intensional nor extensional type theory, but rather for an intermediate theory which we call two-dimensional type theory. Recall that extensional type theory distinguishes itself from intensional type theory by its admission of an equality reflection rule, which states that any two terms of type $A$ which are propositionally equal, are also definitionally equal. The two-dimensional type theory that we will consider admits instances of the equality reflection rule at just those types which are themselves identity types. The effect this has is to collapse the higher-dimensional aspects of the intensional theory, but only above dimension two: and it is this which allows a complete semantics in 2-categories. The leading example of model for our semantics is the groupoid model of [17]; indeed, it plays the same fundamental role for two-dimensional type theory as the Set-based model does for extensional type theory. However, we expect there to be many more examples: on the categorical side, prestack and stack models, which will provide two-dimensional analogues of the presheaf and sheaf models of extensional type theory; and on the type-theoretic side, an E-groupoid model [1], which extends to two dimensions the setoid model of extensional type theory [14]. Once again, the task of describing these models will be deferred to a subsequent paper.

Our hope is that the semantics we describe in this paper will provide a useful guide in setting up more elaborate semantics for intensional type theory: both of the $\omega$-categorical kind outlined above, and of the homotopy-theoretic kind espoused in [2]. Indeed, most of the problematic features of these higher-dimensional semantics are fully alive in the two-dimensional case - in particular, the rather subtle issues regarding stability of structure under substitution - and the analysis we give of them here should prove useful in understanding these more general situations.

The paper is set out as follows. In Section 2, we review the syntax of intensional and extensional Martin-Löf type theory and describe our intermediate two-dimensional theory, $\mathrm{ML}_{2}$. In Sections 3 and 4 we describe a 2-categorical structure built from the syntax of $\mathrm{ML}_{2}$. Section 3 makes use of the non-logical rules of $\mathrm{ML}_{2}$ together with the rules for identity types in order to construct a 2 -category $\mathfrak{C}$ of contexts; a two-dimensional fibration $\mathfrak{T} \rightarrow \mathfrak{C}$ of types over contexts; and a comprehension 2-functor $E: \mathfrak{T} \rightarrow \mathfrak{C}^{\mathbf{2}}$, sending each type-in-context $\Gamma \vdash A$ type to the corresponding dependent projection map 
$(\Gamma, x: A) \rightarrow \Gamma$. So far we have given nothing more than a simple-minded extension of the one-dimensional semantics; the twist is that each dependent projection in our 2categorical model carries the structure of a normal isofibration. This can be seen as the semantic correlate of the Leibniz rule in dependent type theory. Section 4 considers the extra structure imposed on this basic framework by the logical rules of $\mathrm{ML}_{2}$. The identity types are characterised as arrow objects in the slices of the 2-category of contexts; whilst the unit type, dependent sums and dependent products admit description in terms of a notion of weak 2-categorical adjointness which we call retract biadjunction. Where a plain adjunction concerns itself with isomorphisms of hom-sets $\mathbb{C}(F X, Y) \cong \mathbb{D}(X, G Y)$, a retract biadjunction instead requires retract equivalences of hom-categories $\mathbf{C}(F X, Y) \simeq$ $\mathbf{D}(X, G Y)$. In particular, dependent sums and products are characterised as left and right retract biadjoints to weakening. These syntactic investigations lead us to define a notion of model for two-dimensional type theory, this being an arbitrary 2-fibration $\mathfrak{T} \rightarrow \mathfrak{C}$ equipped with the structure outlined above; and the results of Sections 3 and 4 can be summarised as saying that to each type theory $\mathrm{S}$ extending $\mathrm{ML}_{2}$ we may assign a classifying two-dimensional model $\mathbb{C}(\mathrm{S})$. In Section 5 , we provide a converse to this result by showing that to each two-dimensional model $\mathbb{C}$ we can assign a two-dimensional type theory $\mathrm{S}(\mathbb{C})$ which represents the model faithfully. We call this type theory the internal language of $\mathbb{C}$. Finally, we show that these two constructions - classifying model and internal language - give rise to a functorial semantics in the sense of [24]: which is to say that they induce an equivalence between suitably defined categories of two-dimensional type theories, and of two-dimensional models.

Acknowledgements The author thanks the anonymous referees for their helpful suggestions.

\section{INTENSIONAL, EXTENSIONAL AND TWO-DIMENSIONAL TYPE THEORY}

2.1. Intensional type theory. By intensional Martin-Löf type theory, we mean the logical calculus set out in Part II of [25]. In this paper, we consider only the core calculus $\mathrm{ML}_{I}$, with type-formers for dependent sums, dependent products, identity types and the unit type. We now summarise this calculus, partly to fix notation and partly because there are few peculiarities which are worth commenting on. The calculus has four basic forms of judgement: $A$ type (" $A$ is a type"); $a: A$ (" $a$ is an element of the type $A$ "); $A=B$ type (" $A$ and $B$ are definitionally equal types"); and $a=b: A$ (" $a$ and $b$ are definitionally equal elements of the type $A$ "). These judgements may be made either absolutely, or relative to a context $\Gamma$ of assumptions, in which case we write them as

$$
\Gamma \vdash A \text { type, } \quad \Gamma \vdash a: A, \quad \Gamma \vdash A=B \text { type } \quad \text { and } \quad \Gamma \vdash a=b: A
$$

respectively. Here, a context is a list $\Gamma=\left(x_{1}: A_{1}, x_{2}: A_{2}, \ldots, x_{n}: A_{n}\right)$, wherein each $A_{i}$ is a type relative to the context $\left(x_{1}: A_{1}, \ldots, x_{i-1}: A_{i-1}\right)$. There are now some rather natural requirements for well-formed judgements: in order to assert that $a: A$ we must first know that $A$ type; to assert that $A=B$ type we must first know that $A$ type and $B$ type; and so on. We specify intensional Martin-Löf type theory as a collection of inference rules over these forms of judgement. Firstly we have the equality rules, which assert that the two judgement forms $A=B$ type and $a=b: A$ are congruences with respect to all the other operations of the theory; then we have the structural rules, 
which deal with weakening, contraction, exchange and substitution ${ }^{1}$; and finally, the logical rules, which we list in Table 1. Note that we commit the usual abuse of notation in leaving implicit an ambient context $\Gamma$ common to the premisses and conclusions of each rule. We also omit the rules expressing stability under substitution in this ambient context. We will find it convenient to use the following extended forms of the identity elimination and computation rules:

$$
\begin{gathered}
x, y: A, p: \operatorname{ld}_{A}(x, y), \Delta \vdash C(x, y, p) \text { type } \\
\frac{x: A, \Delta[x, x, \mathrm{r}(x) / x, y, p] \vdash d(x): C(x, x, \mathrm{r}(x))}{x, y: A, p: \operatorname{ld}_{A}(x, y), \Delta \vdash \mathrm{J}_{d}(x, y, p): C(x, y, p)} \\
x, y: A, p: \operatorname{ld}_{A}(x, y), \Delta \vdash C(x, y, p) \text { type } \\
x: A, \Delta[x, x, \mathrm{r}(x) / x, y, p] \vdash d(x): C(x, x, \mathrm{r}(x)) \\
\frac{x: A, \Delta[x, x, \mathrm{r}(x) / x, y, p] \vdash \mathrm{J}_{d}(x, x, \mathrm{r}(x))=d(x): C(x, x, \mathrm{r}(x))}{}
\end{gathered}
$$

These rules may be derived from the elimination and computation rules in Table 1 by using the $\Pi$-types to shift the additional contextual parameter $\Delta$ onto the right-hand side of the turnstile.

Notation 2.1.1. We may omit from the premisses of a rule or deduction any hypothesis which may be inferred from later hypotheses of that rule. Where it improves clarity we may omit brackets in function applications, writing $h g f x$ in place of $h(g(f(x)))$, for example. We may drop the subscript $A$ in an identity type $\operatorname{ld}_{A}(a, b)$ where no confusion seems likely to occur. We may write a sum type $\Sigma x: A$. $B(x)$ as $\Sigma(A, B)$, a product type $\Pi x: A . B(x)$ as $\Pi(A, B)$, and a $\lambda$-abstraction $\lambda x . f(x)$ as $\lambda(f)$ (or using our applicative convention, simply $\lambda f$ ). It will occasionally be useful to perform lambda-abstraction at the meta-theoretic level, for instance writing $[x] f(x)$ to denote a term $f$ of the form $x: A \vdash f(x): B(x)$. We may write $\Gamma \vdash a \approx b: A$ to indicate that the type $\Gamma \vdash \operatorname{ld}_{A}(a, b)$ is inhabited, and say that $a$ and $b$ are propositionally equal. We will also make use of vector notation in the style of [4]. Given a context $\Gamma=\left(x_{1}: A_{1}, \ldots, x_{n}: A_{n}\right)$, we may abbreviate a series of judgements:

$$
\vdash a_{1}: A_{1}, \quad \vdash a_{2}: A_{2}\left(a_{1}\right), \quad \ldots \quad \vdash a_{n}: A_{n}\left(a_{1}, \ldots, a_{n-1}\right),
$$

as $\vdash a: \Gamma$, where $a:=\left(a_{1}, \ldots, a_{n}\right)$, and say that $a$ is a global element of $\Gamma$. We may also use this notation to abbreviate sequences of hypothetical elements on the left-hand side of the turnstile; so, for example, we may specify a dependent type in context $\Gamma$ as $x: \Gamma \vdash A(x)$ type. We will also make use of [4]'s notion of telescope. Given $\Gamma$ a context as before, this allows us to abbreviate the series of judgements

$$
\begin{gathered}
x: \Gamma \vdash B_{1}(x) \text { type } \\
x: \Gamma, y_{1}: B_{1} \vdash B_{2}\left(x, y_{1}\right) \text { type } \\
\cdots \\
x: \Gamma, y_{1}: B_{1}, \ldots, y_{m-1}: B_{m-1} \\
\vdash B_{m}\left(x, y_{1}, \ldots y_{m-1}\right) \text { type. }
\end{gathered}
$$

as $x: \Gamma \vdash \Delta(x)$ ctxt, where $\Delta(x):=\left(y_{1}: B_{1}(x), y_{2}: B_{2}\left(x, y_{1}\right), \ldots\right)$. We say that $\Delta$ is a context in context $\Gamma$, or a context dependent upon $\Gamma$, and refer to contexts like $\Delta$ as

\footnotetext{
${ }^{1}$ Note in particular that we take substitution to be a primitive, rather than a derived operation: as done in [20], for instance.
} 
Dependent sum types

$$
\begin{gathered}
\frac{A \text { type } \quad x: A \vdash B(x) \text { type }}{\Sigma x: A \cdot B(x) \text { type }} \Sigma \text {-Form; } \quad \frac{a: A \quad b: B(a)}{\langle a, b\rangle: \Sigma x: A \cdot B(x)} \Sigma \text {-InTro; } \\
\frac{z: \Sigma x: A . B(x) \vdash C(z) \text { type } \quad x: A, y: B(x) \vdash d(x, y): C(\langle x, y\rangle)}{z: \Sigma x: A . B(x) \vdash \mathrm{E}_{d}(z): C(z)} \Sigma \text {-ELim; } \\
\frac{z: \Sigma x: A . B(x) \vdash C(z) \text { type } \quad x: A, y: B(x) \vdash d(x, y): C(\langle x, y\rangle)}{x: A, y: B(x) \vdash \mathrm{E}_{d}(\langle x, y\rangle)=d(x, y): C(\langle x, y\rangle)} \Sigma \text {-COMP. }
\end{gathered}
$$

Unit type

$$
\begin{aligned}
& \frac{\overline{1} \text { type }}{1-F O R M ;} \quad \bar{\star} \text { 1-Intro; } \\
& \frac{z: \mathbf{1} \vdash C(z) \text { type } \quad d: C(\star)}{z: \mathbf{1} \vdash \mathrm{U}_{d}(z): C(z)} \text { 1-ELIM; } \\
& \frac{z: \mathbf{1} \vdash C(z) \text { type } \quad d: C(\star)}{\mathrm{U}_{d}(\star)=d: C(\star)} \text { 1-COMP. }
\end{aligned}
$$

\section{Identity types}

$$
\begin{gathered}
\frac{A \text { type } a, b: A}{\operatorname{ld}_{A}(a, b) \text { type }} \text { Id-Form; } \quad \frac{A \text { type } a: A}{\mathrm{r}(a): \operatorname{ld}_{A}(a, a)} \text { Id-INTro; } \\
\frac{x, y: A, p: \operatorname{ld}_{A}(x, y) \vdash C(x, y, p) \text { type } \quad x: A \vdash d(x): C(x, x, \mathrm{r}(x))}{x, y: A, p: \operatorname{ld}_{A}(x, y) \vdash \mathrm{J}_{d}(x, y, p): C(x, y, p)} \text { Id-ELIM; } \\
\frac{x, y: A, p: \operatorname{ld}_{A}(x, y) \vdash C(x, y, p) \text { type } \quad x: A \vdash d(x): C(x, x, \mathrm{r}(x))}{x: A \vdash \mathrm{J}_{d}(x, x, \mathrm{r}(x))=d(x): C(x, x, \mathrm{r}(x))} \text { Id-COMP. }
\end{gathered}
$$

\section{Dependent product types}

$$
\begin{aligned}
& \frac{A \text { type } \quad x: A \vdash B(x) \text { type }}{\Pi x: A . B(x) \text { type }} \text { П-Form; } \quad \frac{x: A \vdash f(x): B(x)}{\lambda x \cdot f(x): \Pi x: A \cdot B(x)} \Pi \text {-ABS; } \\
& \frac{m: \Pi x: A \cdot B(x)}{y: A \vdash m \cdot y: B(y)} \Pi \text {-APP; } \quad \frac{x: A \vdash f(x): B(x)}{y: A \vdash(\lambda x \cdot f(x)) \cdot y=f(y): B(y)} \Pi-\beta .
\end{aligned}
$$

TABLE 1. Logical rules of intensional Martin-Löf type theory $\left(\mathrm{ML}_{I}\right)$ 
dependent contexts, and to those like $\Gamma$ as closed contexts. Given a dependent context $x: \Gamma \vdash \Delta(x)$ ctxt, we may abbreviate the series of judgements

$$
\begin{gathered}
x: \Gamma \vdash f_{1}(x): B_{1}(x) \\
\ldots \\
x: \Gamma \vdash f_{m}(x): B_{m}\left(x, f_{1}(x), \ldots, f_{m-1}(x)\right),
\end{gathered}
$$

as $x: \Gamma \vdash f(x): \Delta(x)$, and say that $f$ is a dependent element of $\Delta$. We can similarly assign a meaning to the judgements $x: \Gamma \vdash \Delta(x)=\Theta(x)$ ctxt and $x: \Gamma \vdash f(x)=g(x)$ : $\Delta(x)$, expressing the definitional equality of two dependent contexts, and the definitional equality of two dependent elements of a dependent context.

2.2. Extensional type theory. We obtain extensional Martin-Löf type theory $\mathrm{ML}_{E}$ by augmenting the intensional theory with the two equality reflection rules:

$$
\frac{a, b: A \quad \alpha: \operatorname{ld}(a, b)}{a=b: A} \quad \frac{a, b: A \quad \alpha: \operatorname{ld}(a, b)}{\alpha=\operatorname{r}(a): \operatorname{ld}(a, b)}
$$

together with the rule of function extensionality:

$$
\frac{m, n: \Pi(A, B) \quad x: A \vdash m \cdot x=n \cdot x}{m=n: \Pi(A, B)} .
$$

The addition of these three rules yields a type theory which is intuitively simpler, and more natural from the perspective of categorical models, but proof-theoretically unpleasant: we lose the decidability of definitional equality and the decidability of type-checking. Note that if one develops Martin-Löf type theory in a framework admitting higher-order inference rules (such as the Logical Framework of [25]) then the above three rules are equipotent with the definitional $\eta$-rule.

2.3. Two-dimensional type theory. The type theory we investigate in this paper lies between the intensional theory of $\S 2.1$ and the extensional theory of $\S 2.2$. We denote it by $\mathrm{ML}_{2}$, and call it two-dimensional type theory, because as we will see, it has a natural semantics in two-dimensional categories. It is obtained by augmenting intensional type theory with the rules of Tables 2 and 3. These provide restricted versions of the equality reflection rules (Table 2) and the function extensionality rules (Table 3). To motivate the rules in Table 2, we introduce the notion of a discrete type. We say that $\Gamma \vdash A$ type is discrete if the judgements

$$
\begin{aligned}
& \frac{\Gamma \vdash a, b: A \quad \Gamma \vdash p: \operatorname{ld}(a, b)}{\Gamma \vdash a=b: A} \operatorname{Id}_{-R^{2} L_{1}-A} ; \\
& \frac{\Gamma \vdash a, b: A \quad \Gamma \vdash p: \operatorname{ld}(a, b)}{\Gamma \vdash p=\mathrm{r}(a): \operatorname{ld}(a, b)} \operatorname{Id}-\mathrm{REFL}_{2}-A
\end{aligned}
$$

are derivable. Thus the intensional theory says that no types need be discrete; the extensional theory says that all types are discrete; and the two-dimensional theory says that all identity types are discrete. Note that although two-dimensional type theory suffers from the same proof-theoretic deficiencies of the extensional theory, it does so in a less severe manner: indeed, only those types of $\mathrm{ML}_{2}$ in whose construction the identity types have been used will have undecidable definitional equality. As we ascend to higher-dimensional variants of type theory, this undecidability will be pushed further 


$$
\begin{gathered}
\frac{a, b: A \quad p, q: \operatorname{Id}(a, b) \quad \alpha: \operatorname{Id}(p, q)}{p=q: \operatorname{ld}(a, b)} \text { Id-DISC } 1 ; \\
\frac{a, b: A \quad p, q: \operatorname{Id}(a, b) \quad \alpha: \operatorname{Id}(p, q)}{\alpha=\mathrm{r}(p): \operatorname{ld}(p, q)} \mathrm{Id}-\mathrm{DISC}_{2} .
\end{gathered}
$$

TABLE 2. Rules for discrete identity types

$$
\begin{gathered}
\frac{m, n: \Pi(A, B) \quad x: A \vdash p(x): \operatorname{ld}(m \cdot x, n \cdot x)}{\operatorname{ext}(m, n, p): \operatorname{ld}(m, n)} \text { П-EXT; } \\
\frac{m: \Pi(A, B)}{\operatorname{ext}(m, m,[x] \mathrm{r}(m \cdot x))=\mathrm{r}(m): \operatorname{ld}(m, m)} \text { П-EXT-COMP; } \\
\frac{m, n: \Pi(A, B) \quad x: A \vdash p(x): \operatorname{ld}(m \cdot x, n \cdot x)}{x: A \vdash \operatorname{ext}(m, n, p) * x=p(x): \operatorname{ld}(m \cdot x, n \cdot x)} \text { П-EXT-APP. }
\end{gathered}
$$

TABLE 3. Rules for function extensionality

and further up the hierarchy of iterated identity types; but it is only in the limit - which is intensional type theory - that we regain complete decidability.

The necessity of the rules in Table 3 will become clear when we reach $\S 4.5$. We require them in order to obtain a satisfactory notion of two-dimensional categorical model, in which dependent product formation is right adjoint to substitution (in a suitably weak 2 -categorical sense). The first of the rules in Table 3 is a propositional version of the function extensionality principle of $\S 2.2$; whilst the second and the third express coherence properties of the first. To understand the third rule we must first explain the symbol * appearing in it. It is a definable constant which expresses that two propositionally equal elements of a П-type are pointwise propositionally equal. Explicitly, it satisfies the following introduction and computation rules:

$$
\begin{gathered}
\frac{m, n: \Pi(A, B) \quad p: \operatorname{ld}(m, n) \quad a: A}{p * a: \operatorname{ld}(m \cdot a, n \cdot a)} \text { *-INTRO; } \\
\frac{m: \Pi(A, B) \quad a: A}{\mathrm{r}(m) * a=\mathrm{r}(m \cdot a): \operatorname{ld}(m \cdot a, m \cdot a)} \text { *-COMP; }
\end{gathered}
$$

and we may define it by Id-elimination, taking $p * a:=\mathrm{J}_{[x] \mathrm{r}(x \cdot a)}(m, n, p)$.

\section{Categorical models for $\mathrm{ML}_{2}$ : structural aspects}

The remainder of this paper will describe a notion of categorical semantics for $\mathrm{ML}_{2}$. In this section and the following one, we define a syntactic category and enumerate its structure; whilst in Section 5, we consider an arbitrary category endowed with this same structure, and derive from it a type theory incorporating the rules of $\mathrm{ML}_{2}$. This yields 
a semantics which is both complete and sound. In this section, we define the basic syntactic category and look at the structure induced on it by the non-logical rules of $\mathrm{ML}_{2}$. In the next section, we consider the logical rules. As mentioned in the Introduction, the syntactic category we define will in fact be a 2-category, whose objects will be (vectors of) types; whose morphisms will be (vectors) of terms between those types; and whose 2cells will be (vectors of) identity proofs between these terms. The various forms of 2-cell composition will be obtained using the identity elimination rules; whilst the rules for discrete identity types given in Table 2 ensure that these compositions satisfy the 2-category axioms. For basic terminology and notation relating to 2-categories we refer to [23].

3.1. One-dimensional semantics of type dependency. We begin by recalling the construction of a one-dimensional categorical structure from the syntax of a dependent type theory. The presentation we have chosen follows [19] in its use of (full) comprehension categories. There are various other, essentially equivalent, presentations that we could have used: see $[5,6,7,18,29]$ for example. We use comprehension categories because they afford a straightforward passage to a two-dimensional structure.

So suppose given an arbitrary dependently-typed calculus S admitting the same four basic judgement types and the same structural rules as the calculus $\mathrm{ML}_{I}$. We define its category of contexts $\mathcal{C}_{\mathrm{S}}$ to have as objects, contexts $\Gamma, \Delta, \ldots$, in $\mathrm{S}$, considered modulo $\alpha$-conversion and definitional equality (so we identify $\Gamma$ and $\Delta$ whenever $\vdash \Gamma=\Delta$ ctxt is derivable); and as morphisms $\Gamma \rightarrow \Delta$, judgements $x: \Gamma \vdash f(x): \Delta$, considered modulo $\alpha$-conversion and definitional equality (so we identity $f, g: \Gamma \rightarrow \Delta$ whenever $x: \Gamma \vdash$ $f(x)=g(x)$ is derivable). To avoid further repetition, we introduce the convention that any further categorical structures we define should also be interpreted modulo $\alpha$ equivalence and definitional equality. The identity map on $\Gamma$ is given by $x: \Gamma \vdash x: \Gamma$; whilst composition is given by substitution of terms. Note that $\mathcal{C}_{\mathrm{S}}$ has a terminal object, given by the empty context ( ).

For each context $\Gamma$ we now define the category $\mathcal{T}_{\mathrm{S}}(\Gamma)$ of types-in-context- $\Gamma$, whose objects $A$ are judgements $x: \Gamma \vdash A(x)$ type and whose morphisms $A \rightarrow B$ are judgements $x: \Gamma, y: A(x) \vdash f(x, y): B(x)$. Each morphism $f: \Gamma \rightarrow \Delta$ of $\mathcal{C}_{\mathrm{S}}$ induces a functor $\mathcal{T}_{\mathrm{S}}(f): \mathcal{T}_{\mathrm{S}}(\Delta) \rightarrow \mathcal{T}_{\mathrm{S}}(\Gamma)$ which sends a type $A$ in context $\Delta$ to the type $f^{*} A$ in context $\Gamma$ given by $x: \Gamma \vdash A(f(x))$ type. The assignation $f \mapsto \mathcal{T}_{\mathrm{S}}(f)$ is itself functorial in $f$, and so we obtain an indexed category $\mathcal{T}_{\mathrm{S}}(-): \mathcal{C}_{\mathrm{S}}^{\mathrm{op}} \rightarrow$ Cat; which via the Grothendieck construction, we may equally well view as a split fibration $p: \mathcal{T}_{\mathrm{S}} \rightarrow \mathcal{C}_{\mathrm{S}}$. We refer to this as the fibration of types over contexts.

Explicitly, objects of $\mathcal{T}_{\mathrm{S}}$ are pairs $(\Gamma, A)$ of a context and a type in that context; whilst morphisms $(\Gamma, A) \rightarrow(\Delta, B)$ are pairs $(f, g)$ of a context morphism $f: \Gamma \rightarrow \Delta$ together with a judgement $x: \Gamma, y: A(x) \vdash g(x, y): B(f(x))$. The chosen cartesian lifting of a morphism $f: \Gamma \rightarrow \Delta$ at an object $(\Delta, B)$ is given by $(f, \iota):\left(\Gamma, f^{*} B\right) \rightarrow(\Delta, B)$, where $\iota$ denotes the judgement $x: \Gamma, y: B(f x) \vdash y: B(f x)$. Now, for each object $(\Gamma, A)$ of $\mathcal{T}_{\mathrm{S}}$ we have the extended context $(x: \Gamma, y: A(x))$, which we denote by $\Gamma . A$; and we also have the judgement $x: \Gamma, y: A(x) \vdash x: \Gamma$, corresponding to a context morphism $\pi_{A}: \Gamma . A \rightarrow \Gamma$ which we call the dependent projection associated to $A$. In fact, the assignation $(\Gamma, A) \mapsto \pi_{A}$ provides the action on objects of a functor $E: \mathcal{T}_{\mathrm{S}} \rightarrow \mathcal{C}_{\mathrm{S}}^{2}$ (where $\mathbf{2}$ denotes the arrow category $0 \rightarrow 1$ ), whose action on maps sends the morphism 
$(f, g):(\Gamma, A) \rightarrow(\Delta, B)$ of $\mathcal{T}_{\mathrm{S}}$ to the morphism

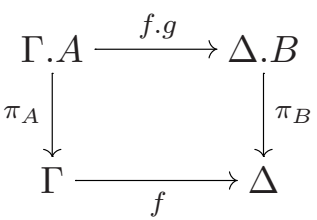

of $\mathcal{C}_{\mathrm{S}}^{2}$, where $f . g$ denotes the judgement $x: \Gamma, y: A \vdash(f(x), g(x, y)): \Delta . B$.

We can make two observations about this functor $E$. Firstly, it is fully faithful, which says that every morphism $h: \Gamma . A \rightarrow \Delta . B$ fitting into a square like (2) is of the form $f . g$ for a unique $(f, g):(\Gamma, A) \rightarrow(\Delta, B)$. Secondly, for a cartesian morphism $(f, \iota):\left(\Gamma, f^{*} B\right) \rightarrow$ $(\Delta, B)$, the corresponding square (2) is a pullback square. Indeed, given an arbitrary commutative square

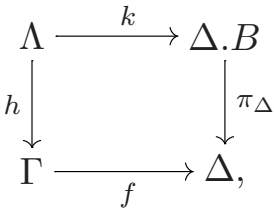

commutativity forces $k$ to be of the form $z: \Lambda \vdash\left(f h z, k^{\prime} z\right): \Delta . B$ for some $z: \Lambda \vdash$ $k^{\prime}(z): B(f h z)$; and so the required factorisation $\Lambda \rightarrow \Gamma \cdot f^{*} B$ is given by the judgement $z: \Lambda \vdash\left(h z, k^{\prime} z\right): \Gamma \cdot f^{*} B$. We may abstract away from the above situation as follows. We define a full split comprehension category (cf. [19]) to be given by a category $\mathcal{C}$ with a specified terminal object, together with a split fibration $p: \mathcal{T} \rightarrow \mathcal{C}$ and a full and faithful functor $E: \mathcal{T} \rightarrow \mathcal{C}^{2}$ rendering commutative the triangle

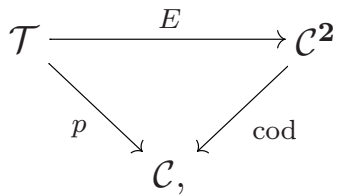

and sending cartesian morphisms in $\mathcal{T}$ to pullback squares in $\mathcal{C}^{2}$. The preceding discussion shows that to any suitable dependent type theory $\mathrm{S}$ we may associate a full split comprehension category $\mathbb{C}(\mathrm{S})$, which we will refer to as the classifying comprehension category of S.

Notation 3.1.1. We will make use of the notation developed above in arbitrary comprehension categories $\left(p: \mathcal{T} \rightarrow \mathcal{C}, E: \mathcal{T} \rightarrow \mathcal{C}^{2}\right)$. Thus we write chosen cartesian liftings as $(f, \iota):\left(\Gamma, f^{*} B\right) \rightarrow(\Delta, B)$, and write the image of $(\Gamma, A) \in \mathcal{T}$ under $E$ as $\pi_{A}: \Gamma . A \rightarrow \Gamma$. We will find it convenient to develop a little more notation. Given $\Gamma \in \mathcal{C}$ and $A \in \mathcal{T}(\Gamma)$, we call a map $a: \Gamma \rightarrow \Gamma$.A satisfying $\pi_{A} a=\operatorname{id}_{\Gamma}$ a global section of $A$, and denote it by $a \in_{\Gamma} A$. Given further a morphism $f: \Delta \rightarrow \Gamma$ of $\mathcal{C}$, we write $f^{*} a \in_{\Delta} f^{*} A$ for the section 
of $\pi_{f^{*} A}$ induced by the universal property of pullback in the following diagram:

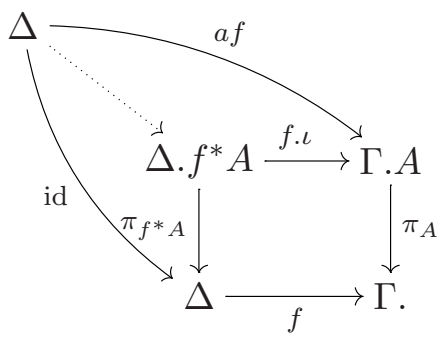

3.2. A 2-category of types. We will now extend the classifying comprehension category $\mathbb{C}(\mathrm{S})$ defined above to a classifying comprehension 2-category. We will not need the full strength of two-dimensional type theory, $\mathrm{ML}_{2}$, for this. Rather, for the rest of this section we fix an arbitrary dependently typed theory $\mathrm{S}$ which admits the structural rules required in the previous subsection together with the identity type rules from Table 1 and the discrete identity rules of Table 2. Our first task will be to construct a 2-category of closed types in S. We will do this by enriching the category $\mathcal{T}_{\mathrm{S}}($ ) of closed types with 2-cells derived from the 2-category of strict internal groupoids in S. A strict internal groupoid in $\mathrm{S}$ is given by a closed type $A_{0}$; a family $A_{1}(x, y)$ of types over $x, y: A_{0}$; and operations of unit, composition and inverse:

$$
\begin{aligned}
x: A_{0} & \vdash \mathrm{id}_{x}: A_{1}(x, x) \\
x, y, z: A_{0}, p: A_{1}(x, y), q: A_{1}(y, z) & \vdash q \circ p: A_{1}(x, z), \\
x, y: A_{0}, p: A_{1}(x, y) & \vdash p^{-1}: A_{1}(y, x),
\end{aligned}
$$

which obey the usual five groupoid axioms up to definitional equality. For instance, the left unit axiom requires that

$$
x, y: A_{0}, p: A_{1}(x, y) \vdash \operatorname{id}_{y} \circ p=p: A_{1}(x, y)
$$

should hold. We will generally write that $\left(A_{0}, A_{1}\right)$ is an internal groupoid in $\mathrm{S}$, leaving the remaining structure understood. Now an internal functor $F:\left(A_{0}, A_{1}\right) \rightarrow\left(B_{0}, B_{1}\right)$ between internal groupoids is given by judgements

$$
\begin{aligned}
x: A_{0} & \vdash F_{0}(x): B_{0} \\
x, y: A_{0}, p: A_{1}(x, y) & \vdash F_{1}(p): B_{1}\left(F_{0} x, F_{0} y\right),
\end{aligned}
$$

subject to the usual two functoriality axioms (up to definitional equality again); whilst an internal natural transformation $\alpha: F \Rightarrow G$ is given by a family of components $x$ : $A_{0} \vdash \alpha(x): B_{1}\left(F_{0} x, G_{0} x\right)$ subject to the (definitional) naturality axiom.

Proposition 3.2.1. The strict groupoids, functors and natural transformations internal to $\mathrm{S}$ form a 2-category $\mathbf{G p d}(\mathrm{S})$ which is locally groupoidal, in the sense that every 2-cell is invertible.

Proof. Recall that for any category $\mathcal{E}$, we can define a 2-category $\operatorname{Gpd}(\mathcal{E})$ of groupoids internal to that category ${ }^{2}$. In particular, we have the 2-category $\operatorname{Gpd}\left(\mathcal{C}_{\mathrm{S}}\right)$ of groupoids internal to the category of contexts of $\mathrm{S}$. Now, each strict internal groupoid $\mathcal{A}$ in $\mathrm{S}$ gives rise to such an internal groupoid $\mathcal{A}^{\prime}$ in $\mathcal{C}_{\mathrm{S}}$ whose object of objects is the context $\left(x: A_{0}\right)$ and whose object of morphisms is the context $\left(x: A_{0}, y: A_{0}, p: A_{1}(x, y)\right)$. We can check

\footnotetext{
${ }^{2}$ One commonly requires the category $\mathcal{E}$ to have pullbacks, but this is inessential.
} 
that internal functors $\mathcal{A} \rightarrow \mathcal{B}$ in $\mathrm{S}$ correspond bijectively with internal functors $\mathcal{A}^{\prime} \rightarrow \mathcal{B}^{\prime}$ in $\mathcal{C}_{\mathrm{S}}$; and that this correspondence extends to the natural transformations between them. Thus we may take $\operatorname{Gpd}(\mathrm{S})$ to be the 2-category whose objects are strict internal groupoids in $\mathrm{S}$, whose hom-categories are given by $\operatorname{Gpd}(\mathrm{S})(\mathcal{A}, \mathcal{B}):=\operatorname{Gpd}\left(\mathcal{C}_{\mathrm{S}}\right)\left(\mathcal{A}^{\prime}, \mathcal{B}^{\prime}\right)$, and whose remaining structure is inherited from $\operatorname{Gpd}\left(\mathcal{C}_{\mathrm{S}}\right)$. Note that every 2-cell of $\operatorname{Gpd}\left(\mathcal{C}_{\mathrm{S}}\right)$ is invertible, so that the same obtains for $\operatorname{Gpd}(\mathrm{S})$

Our method for obtaining the 2-category of closed types will be to construct a functor $\mathcal{T}_{\mathrm{S}}(\mathrm{)} \rightarrow \boldsymbol{G} \mathbf{p d}(\mathrm{S})$, and to lift the 2-cell structure of $\operatorname{Gpd}(\mathrm{S})$ along it.

Proposition 3.2.2. To each closed type $A$ in $\mathrm{S}$ we may assign a strict internal groupoid $\left(A, \operatorname{Id}_{A}\right)$; and the assignation $A \mapsto\left(A, \operatorname{Id}_{A}\right)$ underlies a functor $\mathcal{T}_{\mathrm{S}}() \rightarrow \operatorname{Gpd}(\mathrm{S})$.

Proof. The proof of this result is essentially due to [17]. We repeat it because we will need the details. We first show that $\left(A, \mathrm{Id}_{A}\right)$ has the structure of a strict internal groupoid. For identities, we take $x: A \vdash \mathrm{id}_{x}:=\mathrm{r}(x): \operatorname{ld}(x, x)$. For composition, we require a judgement

$$
x, y, z: A, p: \operatorname{ld}(x, y), q: \operatorname{ld}(y, z) \vdash q \circ p: \operatorname{ld}(x, z) ;
$$

and by Id-elimination on $p$-in the extended form given in equation (1) - it suffices to define this when $y=z$ and $q=r(y)$, for which we take $r(y) \circ p:=p$. Similarly, to give the judgement

$$
x, y: A, p: \operatorname{ld}(x, y) \vdash p^{-1}: \operatorname{ld}(y, x)
$$

providing inverses, it suffices to consider the case $x=y$ and $p=\mathrm{r}(x)$; for which we take $\mathrm{r}(x)^{-1}=\mathrm{r}(x)$. We must now check the five groupoid axioms. The first unitality axiom $\operatorname{id}_{y} \circ p=p$ follows from the Id-computation rule. For the other unitality axiom, it suffices, by discrete identity types, to show that

$$
x, y: A, p: \operatorname{ld}(x, y) \vdash p \circ \mathrm{r}(x) \approx p: \operatorname{ld}(x, y)
$$

holds; and by Id-elimination, it suffices to do this in the case $x=y$ and $p=\mathrm{r}(x)$, for which we have that $\mathrm{r}(x) \circ \mathrm{r}(x)=\mathrm{r}(x)$ as required. Likewise, for the associativity axiom, it suffices to show that

$$
\begin{aligned}
w, x, y, z: A, p: \operatorname{ld}(w, x), q: \operatorname{ld}(x, y), s: \operatorname{ld}(y, z) & \\
& \vdash s \circ(q \circ p) \approx(s \circ q) \circ p: \operatorname{ld}(w, z) ;
\end{aligned}
$$

and again by Id-elimination, it suffices to do this when $y=z$ and $s=\mathrm{r}(y)$, when have that $\mathrm{r}(y) \circ(q \circ p)=q \circ p=(\mathrm{r}(y) \circ q) \circ p$ as required. Note that again, we require the extended form of Id-elimination of equation (1), and in future we will use this without further comment. The invertibility axioms are similar. Suppose now that in addition to $A$ we are given another type $B$ together with a judgement $x: A \vdash f(x): B$ between them. We will extend this to an internal functor $\left(f, f^{\bullet}\right):\left(A, \operatorname{ld}_{A}\right) \rightarrow\left(B, \operatorname{ld}_{B}\right)$. We define the action on hom-types

$$
x, y: A, p: \operatorname{ld}(x, y) \vdash f^{\bullet}(p): \operatorname{ld}(f x, f y)
$$

by Id-elimination on $p$ : for when $x=y$ and $p=\mathrm{r}(x)$, we may take $f^{\bullet}(\mathrm{r}(x)):=\mathrm{r}(f(x))$. We must now check the functoriality axioms. That $\left(f, f^{\bullet}\right)$ preserves identities follows from the Id-computation rule; whilst to to show that it preserves binary composition, it suffices by discrete identity types to show that

$$
x, y, z: A, p: \operatorname{Id}(x, y), q: \operatorname{Id}(y, z) \vdash f^{\bullet}(q \circ p) \approx f^{\bullet}(q) \circ f^{\bullet}(p): \operatorname{Id}(f x, f z)
$$


holds; and this follows by Id-elimination on $q$, since when $y=z$ and $q=r(y)$, we have that $f^{\bullet}(\mathrm{r}(y) \circ p)=f^{\bullet}(p)=\mathrm{r}(f(y)) \circ f^{\bullet}(p)=f^{\bullet}(\mathrm{r}(y)) \circ f^{\bullet}(p)$ as required. We must now check that the assignation $f \mapsto\left(f, f^{\bullet}\right)$ is itself functorial. To show that it preserves identities, we must show that for any closed type $A$,

$$
x, y: A, p: \operatorname{ld}(x, y) \vdash\left(\operatorname{id}_{A}\right)^{\bullet}(p)=p: \operatorname{ld}(x, y)
$$

holds. By discrete identity types, it suffices to show this up to mere propositional equality; and by Id-elimination, we need only do so in the case when $x=y$ and $p=\mathrm{r}(x)$, when we have that $\left(\mathrm{id}_{A}\right)^{\bullet}(\mathrm{r}(x))=\mathrm{r}\left(\mathrm{id}_{A}(x)\right)=\mathrm{r}(x)$ as required. To show that $f \mapsto\left(f, f^{\bullet}\right)$ respects composition, we must show that for maps of closed types $f: A \rightarrow B$ and $g: B \rightarrow C$, the judgement

$$
x, y: A, p: \operatorname{ld}(x, y) \vdash(g f)^{\bullet}(p)=g^{\bullet}\left(f^{\bullet}(p)\right): \operatorname{ld}(g f x, g f y)
$$

holds. Again, it suffices to do this only up to propositional equality, and this only in the case where $x=y$ and $p=\mathrm{r}(x)$; whereupon we have that $(g f)^{\bullet}(\mathrm{r}(x))=\mathrm{r}(g(f(x)))=$ $g^{\bullet}\left(\mathrm{r}(f(x))=g^{\bullet}\left(f^{\bullet}(\mathrm{r}(x))\right.\right.$ as required.

Corollary 3.2.3. The category $\mathcal{T}_{\mathrm{S}}($ ) of closed types in $\mathrm{S}$ may be extended to a locally groupoidal 2-category $\mathfrak{T}_{\mathrm{S}}(\mathrm{)}$ whose 2-cells $\alpha: f \Rightarrow g: A \rightarrow B$ are judgements $x: A \vdash$ $\alpha(x): \operatorname{ld}_{B}(f x, g x)$.

Proof. If we view $\mathcal{T}_{\mathrm{S}}(\mathrm{)})$ as a 2-category with only identity 2-cells, then the functor of the previous proposition may be seen as a 2 -functor $\mathcal{T}_{\mathrm{S}}() \rightarrow \mathbf{G p d}(\mathrm{S})$. We can factorise this 2 -functor as a composite

$$
\mathcal{T}_{\mathrm{S}}() \rightarrow \mathfrak{T}_{\mathrm{S}}(\mathrm{)} \rightarrow \operatorname{Gpd}(\mathrm{S}),
$$

whose first part is bijective on objects and 1-cells and whose second part is fully faithful on 2-cells; and we now define $\mathfrak{T}_{\mathrm{S}}($ ) to be the intermediate 2-category in this factorisation. We must check that this definition agrees with the description of $\mathfrak{T}_{\mathrm{S}}()$ given above. Clearly this is so for the objects and morphisms; whilst for the 2-cells, we must show that for any $f, g: A \rightarrow B$, each judgement $x: A \vdash \alpha(x): \operatorname{ld}_{B}(f x, g x)$ satisfies the axiom for an internal natural transformation $\alpha:\left(f, f^{\bullet}\right) \Rightarrow\left(g, g^{\bullet}\right)$. By discrete identity types, this amounts to validating the judgement

$$
x, y: A, p: \operatorname{ld}_{A}(x, y) \vdash g^{\bullet}(p) \circ \alpha(x) \approx \alpha(y) \circ f^{\bullet}(p): \operatorname{ld}_{B}(f x, g y) ;
$$

and by Id-elimination on $p$, it suffices to do this in the case where $x=y$ and $p=\mathrm{r}(x)$ : for which we have that $g^{\bullet}(\mathrm{r}(x)) \circ \alpha(x)=\mathrm{r}(g(x)) \circ \alpha(x)=\alpha(x)=\alpha(x) \circ \mathrm{r}(f(x))=$ $\alpha(x) \circ f^{\bullet}(\mathrm{r}(x))$, as required.

Corollary 3.2.4. For any context $\Gamma$ in $\mathrm{S}$, the category $\mathcal{T}_{\mathrm{S}}(\Gamma)$ of types-in-context- $\Gamma$ may be extended to a locally groupoidal 2-category $\mathfrak{T}_{\mathrm{S}}(\Gamma)$ wherein 2-cells $\alpha: f \Rightarrow g$ are judgements $x: \Gamma, y: A \vdash \alpha(x, y): \operatorname{ld}_{B}(f(x, y), g(x, y))$.

Proof. We consider the slice theory $\mathrm{S} / \Gamma$, whose closed types are the types of $\mathrm{S}$ in context $\Gamma$. It is easy to see that $S / \Gamma$ admits the same inference rules as $S$ - and in particular has discrete identity types - so that the result follows upon identifying $\mathfrak{T}_{\mathrm{S}}(\Gamma)$ with $\mathfrak{T}_{\mathrm{S} / \Gamma}()$. 
3.3. A 2-category of contexts. In this section, we generalise the construction of the 2 -category of closed types in order to construct a 2-category of contexts. The method will be a direct transcription of the one used in the previous section, but in order for it to make sense, we need to extend the identity type constructor to a "meta-constructor" which operates on entire contexts rather than single types.

Proposition 3.3.1. The following inference rules are definable in $\mathrm{S}$.

$$
\begin{aligned}
& \frac{\Phi \text { ctxt } \quad a, b: \Phi}{\operatorname{ld}_{\Phi}(a, b) \operatorname{ctxt}} \text { Id-FORM'; } \quad \frac{\Phi \text { ctxt } a: \Phi}{\mathrm{r}(a): \operatorname{Id}_{\Phi}(a, a)} \text { Id-INTRO'; } \\
& x, y: \Phi, p: \operatorname{ld}_{\Phi}(x, y), \Delta \vdash \Theta(x, y, p) \text { ctxt } \\
& \frac{x: \Phi, \Delta[x, x, \mathrm{r}(x) / x, y, p] \vdash d(x): \Theta(x, x, \mathrm{r}(x))}{x, y: \Phi, p: \operatorname{Id}_{\Phi}(x, y), \Delta \vdash \mathrm{J}_{d}(x, y, p): \Theta(x, y, p)} \text { Id-ELIM'; } \\
& x, y: \Phi, p: \operatorname{ld}_{\Phi}(x, y), \Delta \vdash \Theta(x, y, p) \text { ctxt } \\
& \frac{x: \Phi, \Delta[x, x, \mathrm{r}(x) / x, y, p] \vdash d(x): \Theta(x, x, \mathrm{r}(x))}{x: \Phi, \Delta[x, x, \mathrm{r}(x) / x, y, p] \vdash \mathrm{J}_{d}(x, x, \mathrm{r}(x))=d(x): \Theta(x, x, \mathrm{r}(x))} \text { Id-COMP'. }
\end{aligned}
$$

In order to prove this result, we will make use of the following well-known consequence of the identity type rules:

Proposition 3.3.2 (The Leibniz rule). Given $A$ type and $x: A \vdash B(x)$ type in $\mathrm{S}$, the following rules are derivable:

$\frac{a_{1}, a_{2}: A \quad p: \operatorname{Id}\left(a_{1}, a_{2}\right) \quad b_{2}: B\left(a_{2}\right)}{p^{*}\left(b_{2}\right): B\left(a_{1}\right)}$ Id-subst; $\quad \frac{a: A \quad b: B(a)}{\mathrm{r}(a)^{*}(b)=b: B(a)}$ Id-SUBsT-COMP.

Proof. By Id-elimination on $p$, it suffices to derive the first rule in the case where $a_{1}=a_{2}$ and $p=\mathrm{r}\left(a_{1}\right)$ : in which case we can take $\mathrm{r}\left(a_{1}\right)^{*}(b):=b$. The second rule now follows from the Id-computation rule.

The key idea behind the proof of Proposition 3.3.1 can be illustrated by considering a context $\Phi=(x: A, y: B(x))$ of length 2 . The corresponding identity context $\mathrm{Id}_{\Phi}$ will be given by

$$
\operatorname{Id}_{\Phi}\left((x, y),\left(x^{\prime}, y^{\prime}\right)\right):=\left(p: \operatorname{ld}_{A}\left(x, x^{\prime}\right), q: \operatorname{Id}_{B(x)}\left(y, p^{*} y^{\prime}\right)\right) .
$$

We use substitution along the first component $p$ to make the second component $q$ typecheck. This can be seen as a type-theoretic analogue of the Grothendieck construction for fibrations. Indeed, it is possible to show that there is a propositional isomorphism between this identity context $\mathrm{Id}_{\Phi}$ and the identity type $\operatorname{ld}_{\Sigma(A, B)}$. Thus in principle it is unnecessary to introduce identity contexts; however, we prefer to do so in order to obtain a cleaner separation between the identity rules and the $\Sigma$-type rules.

Proof of Proposition 3.3.1. The proof has two stages. First, we define the generalised Id-inference rules in the special case where the context $\Phi$ has length 1 ; and then we use these to define them in the general case. We will reduce syntactic clutter by proving our results only in the case where the postcontext $\Delta$ is empty: the reader may readily supply the annotations for the general case. For the first part of the proof, we suppose ourselves given a context $\Phi=(x: A)$ of length 1 . The inference rules Id-FORM' and Id-INTRO' 
for $\Phi$ are just the usual Id-formation and Id-introduction rules for $A$. However, Id-ELIM' corresponds to the following generalised elimination rule:

$$
\frac{x, y: A, p: \operatorname{ld}(x, y) \vdash \Theta(x, y, p) \operatorname{ctxt} \quad x: A \vdash d(x): \Theta(x, x, \mathrm{r}(x))}{x, y: A, p: \operatorname{ld}(x, y) \vdash \mathrm{J}_{d}(x, y, p): \Theta(x, y, p)}
$$

with Id-COMP' stating that $\mathrm{J}_{d}(a, a, \mathrm{r}(a))=d(a)$. We will define the elimination rule by induction on the length $n$ of the context $\Theta$. When $n=0$, this is trivial, and when $n=1$, we use the usual identity elimination rule. So suppose now that we have defined the rule for all contexts $\Theta$ of length $n$, and consider a context $x, y: A, p: \operatorname{ld}(x, y) \vdash \Theta(x, y, p)$ ctxt of length $n+1$. Thus $\Theta$ is of the form

$$
\Theta(x, y, p)=(u: \Lambda(x, y, p), v: D(x, y, p, u))
$$

for some context $\Lambda$ of length $n$ and type $D$. It follows that to make a judgement $x: A \vdash d(x): \Theta(x, x, \mathrm{r}(x))$ is equally well to make a pair of judgements

$$
\begin{aligned}
& x: A \vdash d_{1}(x): \Lambda(x, x, \mathrm{r}(x)) \\
& x: A \vdash d_{2}(x): D\left(x, x, \mathrm{r}(x), d_{1}(x)\right) .
\end{aligned}
$$

By the inductive hypothesis, we may apply the elimination rule (4) for the context $\Lambda$ with eliminating family $d_{1}$ to deduce the existence of a term

$$
x, y: A, p: \operatorname{ld}(x, y) \vdash \mathrm{J}_{d_{1}}(x, y, p): \Lambda(x, y, p),
$$

satisfying $\mathrm{J}_{d_{1}}(x, x, \mathrm{r}(x))=d_{1}(x)$. Now we consider the dependent type

$$
x, y: A, p: \operatorname{ld}(x, y) \vdash C(x, y, p):=D\left(x, y, p, \mathrm{~J}_{d_{1}}(x, y, p)\right) \text { type. }
$$

We have that $C(x, x, \mathrm{r}(x))=D\left(x, x, \mathrm{r}(x), \mathrm{J}_{d_{1}}(x, x, \mathrm{r}(x))\right)=D\left(x, x, \mathrm{r}(x), d_{1}(x)\right)$ and so from (5) we can derive the judgement

$$
x: A \vdash d_{2}(x): C(x, x, \mathrm{r}(x)) .
$$

Now applying the standard Id-elimination rule to (7) and (8) yields a judgement

$$
x, y: A, p: \operatorname{ld}(x, y) \vdash \mathrm{J}_{d_{2}}(x, y, p): D\left(x, y, p, \mathrm{~J}_{d_{1}}(x, y, p)\right)
$$

satisfying $\mathrm{J}_{d_{2}}(x, x, \mathrm{r}(x))=d_{2}(x)$. But to give (6) and (9) is equally well to give a dependent element $x, y: A, p: \operatorname{Id}(x, y) \vdash \mathrm{J}_{d}(x, y, p): \Theta(x, y, p)$; and the respective computation rules for $\mathrm{J}_{d_{1}}$ and $\mathrm{J}_{d_{2}}$ now imply the computation rule for $\mathrm{J}_{d}$. This completes the first part of the proof.

We now construct the generalised inference rules for an arbitrary context $\Phi$. Once again the proof will be by induction, this time on the length of $\Phi$. For the base case, the only context of length 0 is ( ), the empty context. For this, we take the identity context $\mathrm{Id}_{(\text {) }}$ also to be the empty context. The introduction rule is vacuous, whilst the elimination rule requires us to provide, for each closed context $\Theta$ and global element $d: \Theta$, a global element $\mathrm{J}_{d}: \Theta$, satisfying the computation rule $\mathrm{J}_{d}=d: \Theta$. Thus we simply take $\mathrm{J}_{d}:=d$ and are done. Suppose now that we have defined identity contexts for all contexts of length $n$, and consider a context $\Phi=\left(x_{1}: \Lambda, x_{2}: D\left(x_{1}\right)\right)$ of length $n+1$. In order to define $\operatorname{ld}_{\Phi}$, we first apply the inductive hypothesis to $\Lambda$ in order to define its Leibniz rule. Thus given $x: \Lambda \vdash \Upsilon(x)$ ctxt, we may define a judgement

$$
x, y: \Lambda, p: \operatorname{ld}_{\Lambda}(x, y), z: \Upsilon(y) \vdash p^{*}(z): \Upsilon(x),
$$


satisfying $\mathrm{r}(x)^{*}(z)=z: \Upsilon(x)$. The proof is as in Proposition 3.3.2. Now, to give the formation rule for $\mathrm{Id}_{\Phi}$ is equally well to give a judgement

$$
x_{1}: \Lambda, y_{1}: D\left(x_{1}\right), x_{2}: \Lambda, y_{2}: D\left(x_{2}\right) \vdash \operatorname{Id}_{\Phi}\left(x_{1}, y_{1}, x_{2}, y_{2}\right) \text { ctxt },
$$

which we do by setting

$$
\operatorname{Id}_{\Phi}\left(x_{1}, y_{1}, x_{2}, y_{2}\right):=\left(p: \operatorname{ld}_{\Lambda}\left(x_{1}, x_{2}\right), q: \operatorname{ld}_{D\left(x_{1}\right)}\left(y_{1}, p^{*} y_{2}\right)\right) .
$$

Next, to define the introduction rule for $\mathrm{Id}_{\Phi}$ is equally well to give judgements

$$
\begin{aligned}
& x: \Lambda, y: D(x) \vdash r_{1}(x, y): \operatorname{ld}_{\Lambda}(x, x) \\
& x: \Lambda, y: D(x) \vdash r_{2}(x, y): \operatorname{ld}_{D(x)}\left(y, r_{1}(x, y)^{*}(y)\right)
\end{aligned}
$$

which we do by setting $r_{1}(x, y):=\mathrm{r}(x)$ and $r_{2}(x, y):=\mathrm{r}(y)$, where for the second of these we make use of the fact that $\operatorname{Id}_{D(x)}\left(y, \mathrm{r}(x)^{*}(y)\right)=\operatorname{ld}_{D(x)}(y, y)$. In order to define the elimination rule for $\mathrm{Id}_{\Phi}$, we first define a context dependent on $x_{1}, x_{2}: \Lambda$ and $p$ : $\operatorname{ld}_{\Lambda}\left(x_{1}, x_{2}\right)$ by

$$
\Delta\left(x_{1}, x_{2}, p\right):=\left(y_{1}: D\left(x_{1}\right), y_{2}: D\left(x_{2}\right), q: \operatorname{ld}_{D\left(x_{1}\right)}\left(y_{1}, p^{*} y_{2}\right)\right) .
$$

We may then write the premisses of the elimination rule for $\operatorname{ld}_{\Phi}$ as:

$$
x_{1}, x_{2}: \Lambda, p: \operatorname{Id}_{\Lambda}\left(x_{1}, x_{2}\right), z: \Delta\left(x_{1}, x_{2}, p\right) \vdash \Theta\left(x_{1}, x_{2}, p, z\right) \text { ctxt }
$$

and

$$
x: \Lambda, y: D(x) \vdash d(x, y): \Theta(x, x, \mathrm{r}(x), y, y, \mathrm{r}(y)) .
$$

We would like to apply the elimination rule for $\operatorname{ld}_{\Lambda}$ (with postcontext $\Delta$ ) to equation (10). In order to do so, we need to exhibit a generating family

$$
x: \Lambda, z: \Delta(x, x, \mathrm{r}(x)) \vdash d^{\prime}(x, z): \Theta(x, x, \mathrm{r}(x), z) ;
$$

which is equivalently a family

$$
x: \Lambda, y_{1}, y_{2}: D(x), q: \operatorname{ld}_{D(x)}\left(y_{1}, y_{2}\right) \vdash d^{\prime}\left(x, y_{1}, y_{2}, q\right): \Theta\left(x, x, \mathrm{r}(x), y_{1}, y_{2}, q\right)
$$

since we have that $\mathrm{r}(x)^{*}\left(y_{2}\right)=y_{2}$. But we may obtain such a family by applying the generalised elimination rule (4) for $\operatorname{ld}_{D(x)}$ to the dependent context

$$
x: \Lambda, y_{1}, y_{2}: D(x), q: \operatorname{ld}_{D(x)}\left(y_{1}, y_{2}\right) \vdash \Theta\left(x, x, \mathrm{r}(x), y_{1}, y_{2}, q\right) \operatorname{ctxt}
$$

with eliminating family (11). This yields a judgement (12) as required, whilst the computation rule says that $d^{\prime}(x, y, y, \mathrm{r}(y))=d(x, y)$. Now applying the elimination rule for $\mathrm{Id}_{\Lambda}$ to (10) and (12) yields a judgement

$$
x_{1}, x_{2}: \Lambda, p: \operatorname{Id}_{\Lambda}\left(x_{1}, x_{2}\right), z: \Delta\left(x_{1}, x_{2}, p\right) \vdash \quad \mathrm{J}_{d^{\prime}}\left(x_{1}, x_{2}, p, z\right): \Theta\left(x_{1}, x_{2}, p, z\right),
$$

of the correct form to provide the conclusion of the elimination rule for $\mathrm{Id}_{\Phi}$. From the computation rule for $\mathrm{Id}_{\Lambda}$, this will satisfy $\mathrm{J}_{d^{\prime}}(x, x, \mathrm{r}(x), z)=d^{\prime}(x, z)$, and so in particular, we obtain that

$$
\mathrm{J}_{d^{\prime}}(x, x, \mathrm{r}(x), y, y, \mathrm{r}(y))=d^{\prime}(x, y, y, \mathrm{r}(y))=d(x, y)
$$

which gives us the computation rule for $\operatorname{ld}_{\Phi}$. 
Using Proposition 3.3.1 we can now construct the 2-category of contexts in S by mimicking the developments of $§ 3.2$. We first define a strict groupoid context in $\mathrm{S}$ to be given by a context $\Gamma_{0}$ together with a dependent family $x, y: \Gamma_{0} \vdash \Gamma_{1}(x, y)$ ctxt of homcontexts, and operations of unit, composition and inverse satisfying the groupoid axioms as before. It is still the case that any groupoid context induces an internal groupoid object in the category of contexts $\mathcal{C}_{\mathrm{S}}$; and so with the obvious definition of functor and natural transformation, we obtain a 2-category $\operatorname{GpdCtxt}(\mathrm{S})$ of groupoid contexts in S. Following Proposition 3.2.2, we now define a functor $\mathcal{C}_{\mathrm{S}} \rightarrow \operatorname{GpdCtxt}(\mathrm{S})$ sending $\Gamma$ to $\left(\Gamma, \mathrm{Id}_{\Gamma}\right)$. A small subtlety we must check in order for this to go through is that $\mathrm{S}$ has not only discrete identity types, but also discrete identity contexts; and this follows by a straightforward induction on the length of a context. Thereafter, the argument of Proposition 3.2.3 carries over to give:

Corollary 3.3.3. The category $\mathcal{C}_{\mathrm{S}}$ of contexts in $\mathrm{S}$ may be extended to a locally groupoidal 2 -category $\mathfrak{C}_{\mathrm{S}}$ whose 2 -cells $\alpha: f \Rightarrow g: \Gamma \rightarrow \Delta$ are judgements $x: \Gamma \vdash \alpha(x): \operatorname{ld}_{\Delta}(f x, g x)$.

We end this section with a simple observation:

Proposition 3.3.4. The 2 -category $\mathfrak{C}_{\mathrm{S}}$ has a 2-terminal object given by the empty context ( ).

Proof. It is clear that every context $\Gamma$ admits a unique morphism !: $\Gamma \rightarrow($ ), which makes ( ) a terminal object. For it to be 2-terminal, we must also show that for any 2-cell $\alpha: ! \Rightarrow !: \Gamma \rightarrow\left(\right.$ ) we have $\alpha=\mathrm{id}_{!}$. But this follows because we defined $\left.\operatorname{ld}_{(\mathrm{l}}\right):=(\mathrm{)}$ in the proof of Proposition 3.3.1.

3.4. A 2-fibration of types over contexts. The next stage in our development will be to extend the fibration of types over contexts to a 2-fibration of types over contexts. In $\S 3.1$, we built the one-dimensional fibration by first defining an indexed category of types over contexts, and then applying the Grothendieck construction. In the twodimensional case it turns out that the indexed 2-category of types over contexts has a structure so elaborate (it is given by a trihomomorphism $\mathfrak{T}_{\mathrm{S}}(-)$ : $\mathfrak{C}_{\mathrm{S}}^{\mathrm{coop}} \rightarrow$ Gray, where Gray is the tricategory of 2-categories, 2-functors, pseudo-natural transformations, and modifications; see [11]) that it is that it is significantly less work to construct the associated 2-fibration directly. We begin by recalling from [13] the definition of 2-fibration. Of the several equivalent formulations given there, the most convenient for our purposes is the following:

Definition 3.4.1. (cf. [13, Theorem 2.8]) Let $\mathbf{E}$ and $\mathbf{B}$ be 2-categories. We say that a 2 -functor $p: \mathbf{E} \rightarrow \mathbf{B}$ is a cloven 2-fibration if the following four conditions are satisfied:

(i) The underlying ordinary functor of $p$ is a cloven fibration of categories;

(ii) Each cartesian 1-cell $f: y \rightarrow z$ of $\mathbf{E}$ has the following two-dimensional universal property: that whenever we are given a 2-cell $\alpha: g \Rightarrow h: x \rightarrow z$ of $\mathbf{E}$ together with a factorisation

$$
p(\alpha)=p(x) \stackrel{\frac{k}{\Downarrow \gamma}}{\underset{l}{\Downarrow}} p(y) \stackrel{p(f)}{\longrightarrow} p(z),
$$


we may lift this to a unique factorisation

$$
\alpha=x \overbrace{l_{l^{\prime}}^{k_{\gamma^{\prime}}^{\prime}}}^{k^{\prime}} y \stackrel{f}{\longrightarrow} z
$$

satisfying $p\left(\gamma^{\prime}\right)=\gamma$.

(iii) For each $x, y \in \mathbf{E}$, the induced functor $p_{x, y}: \mathbf{E}(x, y) \rightarrow \mathbf{B}(p x, p y)$ is a cloven fibration of categories;

(iv) For each $x, y, z \in \mathbf{E}$ and $f: x \rightarrow y$, the functor $(-) \cdot f: \mathbf{E}(y, z) \rightarrow \mathbf{E}(x, z)$ preserves cartesian liftings of 2-cells.

We say further that a cloven 2-fibration is globally split if its underlying fibration of categories in (i) is a split fibration.

We will now show that the split fibration $p: \mathcal{T}_{\mathrm{S}} \rightarrow \mathcal{C}_{\mathrm{S}}$ of types over contexts extends to a globally split 2-fibration $p: \mathfrak{T}_{\mathrm{S}} \rightarrow \mathfrak{C}_{\mathrm{S}}$. The first step will be to construct the total 2-category $\mathfrak{T}_{\mathrm{S}}$. Before doing this we prove a useful lemma.

Lemma 3.4.2. For a dependent projection $\pi_{A}: \Gamma . A \rightarrow \Gamma$ of $\mathfrak{C}_{\mathrm{S}}$, its lifting to an internal functor $\left(\pi_{A}, \pi_{A}^{\bullet}\right)$, as defined in Proposition 3.2.2, satisfies

$$
(x, y),\left(x^{\prime}, y^{\prime}\right): \Gamma \cdot A,(p, q): \operatorname{ld}_{\Gamma \cdot A}\left((x, y),\left(x^{\prime}, y^{\prime}\right)\right) \vdash \pi_{A} \bullet(p, q)=p: \operatorname{Id}_{\Gamma}\left(x, x^{\prime}\right) .
$$

Proof. By discrete identity types, it suffices to show that $\pi_{A} \bullet(p, q) \approx p$; and by Idelimination on $\Gamma$. $A$, we need only consider the case where $x=x^{\prime}, y=y^{\prime}, p=\mathrm{r}(x)$ and $q=r(y)$. But here, by definition of $\pi_{A} \bullet$, we have $\pi_{A}^{\bullet}(\mathrm{r}(x), \mathrm{r}(y))=\mathrm{r}\left(\pi_{A}(x, y)\right)=\mathrm{r}(x)$ as required.

Proposition 3.4.3. The category $\mathcal{T}_{\mathrm{S}}$ defined in $\S 3.1$ extends to a locally groupoidal 2category $\mathfrak{T}_{\mathrm{S}}$ whose 2 -cells $(\alpha, \beta):(f, g) \Rightarrow\left(f^{\prime}, g^{\prime}\right):(\Gamma, A) \rightarrow(\Delta, B)$ are given by pairs of judgements

$$
\begin{gathered}
x: \Gamma \vdash \alpha(x): \operatorname{Id}_{\Delta}\left(f x, f^{\prime} x\right) \\
x: \Gamma, y: A(x) \vdash \beta(x, y): \operatorname{ld}_{B(f x)}\left(g(x, y), \alpha(x)^{*}\left(g^{\prime}(x, y)\right)\right) .
\end{gathered}
$$

Proof. If we view $\mathcal{T}_{\mathrm{S}}$ as a 2-category with only identity 2-cells, then the functor $E: \mathcal{T}_{\mathrm{S}} \rightarrow$ $\mathcal{C}_{\mathrm{S}}^{2}$ defined in $\S 3.1$ may be viewed as a 2 -functor $\mathcal{T}_{\mathrm{S}} \rightarrow \mathfrak{C}_{\mathrm{S}}^{2}$. We can factorise this 2 -functor as a composite

$$
\mathcal{T}_{\mathrm{S}} \rightarrow \mathfrak{T}_{\mathrm{S}} \rightarrow \mathfrak{C}_{\mathrm{S}}^{2}
$$

whose first part is bijective on objects and 1-cells and whose second part is bijective on 2-cells. We claim that the intermediate 2-category is the $\mathfrak{T}_{\mathrm{S}}$ of the Proposition. Clearly it has the correct objects and 1-cells, whilst for the 2-cells, we must show that given maps $(f, g),\left(f^{\prime}, g^{\prime}\right):(\Lambda, A) \rightarrow(\Delta, B)$ of $\mathcal{T}_{\mathrm{S}}$, pairs of judgements as in (13) are in bijection with 
diagrams

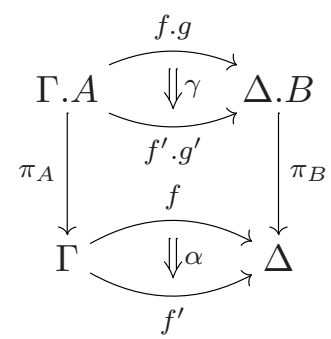

in $\mathfrak{C}_{\mathrm{S}}$ satisfying $\pi_{B} \gamma=\alpha \pi_{A}$. For a diagram like (15), the 2-cell $\gamma: f . g \Rightarrow f^{\prime} \cdot g^{\prime}$ correspondsby the definition of $\operatorname{ld}_{\Delta . B}$ given in Proposition 3.3.1 - to a pair of judgements

$$
\begin{aligned}
& x: \Gamma, y: A(x) \vdash \gamma_{1}(x, y): \operatorname{Id}_{\Delta}\left(f x, f^{\prime} x\right) \\
& x: \Gamma, y: A(x) \vdash \gamma_{2}(x, y): \operatorname{ld}_{B(f x)}\left(g(x, y), \gamma_{1}(x, y)^{*}\left(g^{\prime}(x, y)\right)\right),
\end{aligned}
$$

whilst the equality $\pi_{B} \gamma=\alpha \pi_{A}$ corresponds to the validity of the judgement

$$
x: \Gamma, y: A(x) \vdash \alpha(x)=\pi_{B}^{\bullet}(\gamma(x, y)): \operatorname{ld}_{\Delta}\left(f x, f^{\prime} x\right) .
$$

But by Lemma 3.4.2, we have $\pi_{B} \bullet(\gamma(x, y))=\gamma_{1}(x, y)$, so that $\alpha(x)=\gamma_{1}(x, y)$, and we may identify (16) with (13) upon taking $\beta:=\gamma_{2}$.

Corollary 3.4.4. The fully faithful functor $E: \mathcal{T}_{\mathrm{S}} \rightarrow \mathcal{C}_{\mathrm{S}}^{2}$ of $\S 3.1$ extends to a 2-fully faithful (i.e., bijective on 1- and 2-cells) 2-functor $E: \mathfrak{T}_{\mathrm{S}} \rightarrow \mathfrak{C}_{\mathrm{S}}^{2}$.

Proof. We take $E$ to be the second half of the factorisation in (14).

We now define $p: \mathfrak{T}_{\mathrm{S}} \rightarrow \mathfrak{C}_{\mathrm{S}}$ to be the composite of the 2-functor $E$ of the previous Proposition with the codomain 2 -functor $\mathfrak{C}_{\mathrm{S}}^{2} \rightarrow \mathfrak{C}_{\mathrm{S}}$; explicitly, $p$ is the 2 -functor sending $(\Gamma, A)$ to $\Gamma,(f, g)$ to $f$ and $(\alpha, \beta)$ to $\alpha$. We intend to show that $p$ is a (globally split) 2 -fibration; and will do so by making using of two further properties of the 2-functor $E: \mathfrak{T}_{\mathrm{S}} \rightarrow \mathfrak{C}_{\mathrm{S}}^{\mathbf{2}}$. The first of these generalises directly the one-dimensional situation described in $§ 3.1$. Its proof is less straightforward than one might think.

Proposition 3.4.5. For each $(\Delta, B) \in \mathfrak{T}_{\mathrm{S}}$ and $f: \Gamma \rightarrow \Delta$ in $\mathfrak{C}_{\mathrm{S}}$, the following pullback square in $\mathfrak{C}_{\mathrm{S}}$ is also a 2-pullback:

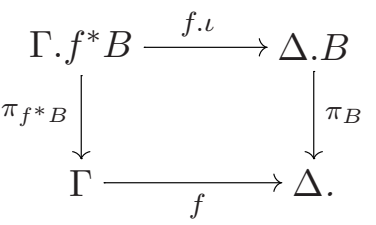

Proof. We begin by introducing a piece of local notation: for the duration of this proof, we will write applications of the Leibniz rule as

$$
\frac{a_{1}, a_{2}: A \quad p: \operatorname{ld}\left(a_{1}, a_{2}\right) \quad b_{2}: B\left(a_{2}\right)}{\operatorname{subst}_{B}\left(p, b_{2}\right): B\left(a_{1}\right)} \text { Id-SUBst. }
$$

We do this in order to make explicit the family $B$ in which substitution is occurring. Now, to say that (17) is not just a pullback but also a 2-pullback is to say that, whenever 
we are given maps $h, k: \Lambda \rightarrow \Gamma \cdot f^{*} B$ and 2-cells

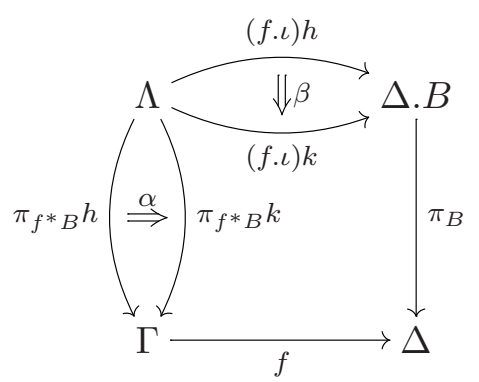

in $\mathfrak{C}_{\mathrm{S}}$ satisfying $f \alpha=\pi_{B} \beta$, we can find a unique 2-cell $\gamma: h \Rightarrow k: \Lambda \rightarrow \Gamma . f^{*} B$ satisfying $\pi_{f^{*} B} \circ \gamma=\alpha$ and $f . \iota \circ \gamma=\beta$. In order to show this, we will first need to understand how $f . \iota$ lifts to an internal functor

$$
\left(f . \iota,(f . \iota)^{\bullet}\right):\left(\Gamma \cdot f^{*} B, \operatorname{ld}_{\Gamma . f^{*} B}\right) \rightarrow\left(\Delta . B, \operatorname{ld}_{\Delta . B}\right) .
$$

So suppose given elements $\left(x_{1}, y_{1}\right)$ and $\left(x_{2}, y_{2}\right): \Gamma . f^{*} B$; now a typical element $(p, q)$ : $\operatorname{Id}_{\Gamma . f^{*} B}\left(\left(x_{1}, x_{2}\right),\left(y_{1}, y_{2}\right)\right)$ is given by a pair of judgements

$$
p: \operatorname{ld}_{\Gamma}\left(x_{1}, y_{1}\right) \quad \text { and } \quad q: \operatorname{ld}_{B\left(f x_{1}\right)}\left(x_{2}, \operatorname{subst}_{f * B}\left(p, y_{2}\right)\right) .
$$

This is sent by $(f . \iota)^{\bullet}$ to some element $(u, v): \operatorname{ld}_{\Delta . B}\left(\left(f x_{1}, y_{1}\right),\left(f y_{1}, y_{2}\right)\right)$, which is equally well a pair of judgements

$$
u: \operatorname{Id}_{\Gamma}\left(f x_{1}, f y_{1}\right) \quad \text { and } \quad v: \operatorname{ld}_{B\left(f x_{1}\right)}\left(x_{2}, \operatorname{subst}_{B}\left(u, y_{2}\right)\right) .
$$

Since we have $\pi_{B} \circ f . \iota=f \circ \pi_{f^{*} B}$, we have by Lemma 3.4.2 that

$$
u=\pi_{B}^{\bullet}(u, v)=\left(\pi_{\mathcal{B}} \circ f . \iota\right)^{\bullet}(p, q)=\left(f \circ \pi_{f^{*} B}\right)^{\bullet}(p, q)=f^{\bullet}(p)
$$

and so it remains only to describe $v$. We will do this by reduction to a special case. Suppose that we have $x_{2}=\operatorname{subst}_{f^{*} B}\left(p, y_{2}\right)$ and $q=\mathrm{r}\left(\operatorname{subst}_{f^{*} B}\left(p, y_{2}\right)\right)$. We denote the corresponding $v$ by

$$
\theta\left(p, y_{2}\right): \operatorname{Id}_{B\left(f x_{1}\right)}\left(\operatorname{subst}_{f^{*} B}\left(p, y_{2}\right), \operatorname{subst}_{B}\left(f \bullet(p), y_{2}\right)\right) .
$$

Note that in the case where $x_{1}=y_{1}$ and $p=\mathrm{r}\left(x_{1}\right)$, we have by Id-computation that $\theta\left(\mathrm{r}\left(x_{1}\right), y_{2}\right)=\mathrm{r}\left(y_{2}\right)$. We now use (21) to describe the general case. We claim that given $p$ and $q$ as in (19), the corresponding $v$ as in (20) satisfies

$$
v=\theta\left(p, y_{2}\right) \circ q: \operatorname{Id}_{B\left(f x_{1}\right)}\left(x_{2}, \operatorname{subst}_{B}\left(f^{\bullet}(p), y_{2}\right)\right) .
$$

Now, by discrete Id-types, it suffices to show this up to propositional equality; and by Id-elimination on $\Gamma$. $f^{*} B$, this only in the case where $x_{1}=y_{1}, p=\mathrm{r}\left(x_{1}\right), x_{2}=y_{2}$ and $q=\mathrm{r}\left(x_{2}\right)$. Here, by definition of $(f . \iota)^{\bullet}$ and Id-computation, we have on the one hand that $v=\mathrm{r}\left(x_{2}\right)$; but on the other that $\theta\left(\mathrm{r}\left(x_{1}\right), x_{2}\right) \circ \mathrm{r}\left(x_{2}\right)=\mathrm{r}\left(x_{2}\right) \circ \mathrm{r}\left(x_{2}\right)=\mathrm{r}\left(x_{2}\right)$ as required. This completes the proof of the claim.

We are now ready to show that (17) is a 2-pullback. So suppose given maps $h, k: \Lambda \rightarrow$ $\Gamma . f^{*} B$ and 2-cells $\alpha, \beta$ as in (18). To give $h$ is to give judgements $x: \Lambda \vdash h_{1}(x): \Gamma$ and $x: \Lambda \vdash h_{2}(x): B\left(f h_{1} x\right)$-and correspondingly for $k$-whilst to give $\alpha$ and $\beta$ as in (18) 
satisfying $f \alpha=\pi_{B} \beta$ is to give judgements

$$
\begin{aligned}
& x: \Lambda \vdash \alpha(x): \operatorname{ld}_{\Gamma}\left(h_{1} x, k_{1} x\right) \\
& x: \Lambda \vdash \beta_{1}(x): \operatorname{ld}_{\Delta}\left(f h_{1} x, f k_{1} x\right) \\
& x: \Lambda \vdash \beta_{2}(x): \operatorname{ld}_{B\left(f h_{1} x\right)}\left(h_{2} x, \operatorname{subst}_{B}\left(\beta_{1} x, k_{2} x\right)\right)
\end{aligned}
$$

satisfying

$$
x: \Lambda \vdash f^{\bullet}(\alpha x)=\pi_{B}^{\bullet}\left(\beta_{1} x, \beta_{2} x\right): \operatorname{ld}_{\Delta}\left(f h_{1} x, f k_{1} x\right) .
$$

By Lemma 3.4.2, we have that $\pi_{B} \bullet\left(\beta_{1} x, \beta_{2} x\right)=\beta_{1}(x)$; and so to give (18) satisfying $f \alpha=\pi_{B} \beta$ is equally well to give a pair of judgements

$$
\begin{aligned}
& x: \Lambda \vdash \alpha(x): \operatorname{Id}_{\Gamma}\left(h_{1} x, k_{1} x\right) \\
\text { and } \quad & x: \Lambda \vdash \beta_{2}(x): \operatorname{Id}_{B\left(f h_{1} x\right)}\left(h_{2} x, \operatorname{subst}_{B}\left(f^{\bullet} \alpha x, k_{2} x\right)\right) .
\end{aligned}
$$

From this we are required to find a unique 2-cell $\gamma: h \Rightarrow k: \Lambda \rightarrow \Gamma \cdot f^{*} B$ satisfying $\pi_{f^{*} B} \circ \gamma=\alpha$ and $(f . \iota) \circ \gamma=\beta$; which is equally well a pair of judgements

$$
\begin{aligned}
& x: \Lambda \vdash \gamma_{1}(x): \operatorname{Id}_{\Gamma}\left(h_{1} x, k_{1} x\right) \\
\text { and } \quad & x: \Lambda \vdash \gamma_{2}(x): \operatorname{ld}_{B\left(f h_{1} x\right)}\left(h_{2} x, \operatorname{subst}_{f^{*} B}\left(\gamma_{1} x, k_{2} x\right)\right)
\end{aligned}
$$

satisfying $\left(\pi_{f^{*} B}\right)^{\bullet}\left(\gamma_{1} x, \gamma_{2} x\right)=\alpha(x)$ and $(f . \iota)^{\bullet}\left(\gamma_{1} x, \gamma_{2} x\right)=\left(f^{\bullet} \alpha x, \beta_{2} x\right)$. Now by Lemma 3.4.2, we have $\left(\pi_{f^{*} B}\right)^{\bullet}\left(\gamma_{1} x, \gamma_{2} x\right)=\gamma_{1}(x)$, whence we must take $\gamma_{1}:=\alpha$; whilst from our investigations above, we have

$$
(f . \iota)^{\bullet}\left(\gamma_{1} x, \gamma_{2} x\right)=(f . \iota)^{\bullet}\left(\alpha x, \gamma_{2} x\right)=\left(f^{\bullet}(\alpha x), \theta\left(\alpha x, k_{2} x\right) \circ \gamma_{2}(x)\right)
$$

which tells us that we must have $\gamma_{2}(x):=\theta\left(\alpha x, k_{2} x\right)^{-1} \circ \beta_{2}(x)$. Uniqueness of $\gamma$ follows easily.

The second property of $E$ we consider has no one-dimensional analogue, as it involves the inherently 2-categorical notion of isofibration:

Definition 3.4.6. Let $\mathbf{K}$ be a 2-category. A morphism $p: X \rightarrow Y$ in $\mathbf{K}$ is said to be a cloven isofibration if for every invertible 2-cell

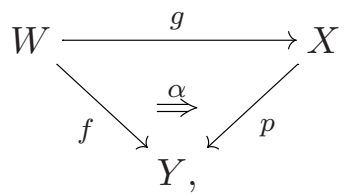

we are given a choice of 1-cell $s_{\alpha}: W \rightarrow X$ and 2-cell $\sigma_{\alpha}: s_{\alpha} \Rightarrow g$ satisfying $p \circ s_{\alpha}=f$ and $p \circ \sigma_{\alpha}=\alpha$; and these choices are natural in $W$, in the sense that given further $k: W^{\prime} \rightarrow W$, we have $s_{\alpha k}=s_{\alpha} \circ k$ and $\sigma_{\alpha k}=\sigma_{\alpha} \circ k$. A cloven isofibration is said to be normal if for any $g: W \rightarrow X$, we have $s_{\operatorname{id}_{p g}}=g$ and $\sigma_{\operatorname{id}_{p g}}=\mathrm{id}_{g}$.

Proposition 3.4.7. Every dependent projection $\pi_{B}: \Delta . B \rightarrow \Delta$ in $\mathfrak{C}_{\mathrm{S}}$ may be equipped with the structure of a normal isofibration.

Proof. Suppose given an invertible 2-cell

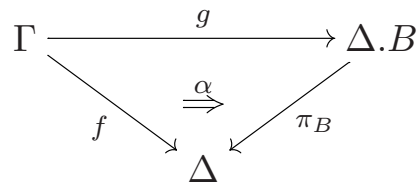


of $\mathfrak{C}_{\mathrm{S}}$. We must find a 1-cell $s_{\alpha}: \Gamma \rightarrow \Delta . B$ and 2-cell $\sigma_{\alpha}: s_{\alpha} \Rightarrow g$ satisfying $\pi_{B} \circ s_{\alpha}=f$ and $\pi_{B} \circ \sigma_{\alpha}=\alpha$. Now, to give a 2-cell as in (23) is equally well to give judgements

$$
\begin{array}{ll}
x: \Gamma \vdash f(x): \Delta, & x: \Gamma \vdash g_{1}(x): \Delta, \\
x: \Gamma \vdash g_{2}(x): B\left(g_{1} x\right), & x: \Gamma \vdash \alpha(x): \operatorname{ld}\left(f x, g_{1} x\right) .
\end{array}
$$

So we may take $s_{\alpha}: \Gamma \rightarrow \Delta$.B to be given by the pair of judgements

$$
x: \Gamma \vdash f(x): \Delta \quad \text { and } \quad x: \Gamma \vdash(\alpha x)^{*}\left(g_{2} x\right): B(f x),
$$

and take $\sigma_{\alpha}: s_{\alpha} \Rightarrow g$ to be given by the pair of judgements

$$
\begin{gathered}
x: \Gamma \vdash \alpha(x): \operatorname{Id}\left(f x, g_{1} x\right) \\
x: \Gamma, y: A(x) \vdash \operatorname{r}\left((\alpha x)^{*}\left(g_{2} x\right)\right): \operatorname{Id}\left((\alpha x)^{*}\left(g_{2} x\right),(\alpha x)^{*}\left(g_{2} x\right)\right) .
\end{gathered}
$$

Given further $k: \Lambda \rightarrow \Gamma$, the equalities $s_{\alpha k}=s_{\alpha} \circ k$ and $\sigma_{\alpha k}=\sigma_{\alpha} \circ k$ correspond precisely to the stability of (24) and (25) under substitution in $x$. Thus $\pi_{B}$ is a cloven isofibration; and it remains to check normality. But when $\alpha$ is an identity 2-cell we have $f(x)=g_{1}(x)$ and $\alpha(x)=\mathrm{r}\left(g_{1}(x)\right)$ and so by the Leibniz computation rule, (24) reduces to $g$ and (25) to $\mathrm{id}_{g}$ as required.

We will refer to the isofibration structure described in Proposition 3.4.7 as the canonical isofibration structure on a dependent projection.

Remark 3.4.8. Proposition 3.4.7 provides a link between the 2-categorical semantics of this paper and the homotopy-theoretic semantics espoused by Awodey and Warren in [2]. The key idea of that paper is that a suitable environment for modelling intensional type theory should be a category equipped with a weak factorisation system $(\mathcal{L}, \mathcal{R})$ (in the sense of [3]) whose right-hand class of maps $\mathcal{R}$ is used to model dependent projections. Now, any finitely complete 2-category carries a weak factorisation system $(\mathcal{L}, \mathcal{R})$ wherein $\mathcal{R}$ is the class of normal isofibrations; it forms one half of what [8, Section 4] calls the "dual of the natural model structure on a 2-category". Thus our two-dimensional semantics fits naturally into the framework outlined in [2].

This result can also be seen as a special case of a result obtained in [9]. The main result of that paper is that the classifying category of any intensional type theory may be equipped with a weak factorisation system whose right class of maps is generated by the dependent projections; and it is shown (Lemma 13) that the maps in this right class are "type-theoretic normal isofibrations". Our Proposition 3.4.7 can be seen as a two-dimensional collapse of this result.

Using Propositions 3.4.5 and 3.4.7, we may now show that:

Proposition 3.4.9. The 2-functor $p: \mathfrak{T}_{\mathrm{S}} \rightarrow \mathfrak{C}_{\mathrm{S}}$ is a globally split 2-fibration.

Proof. We check the four clauses in Definition 3.4.1. Clause (i) is immediate, since the underlying ordinary functor of $p: \mathfrak{T}_{\mathrm{S}} \rightarrow \mathfrak{C}_{\mathrm{S}}$ is the split fibration $p: \mathcal{T}_{\mathrm{S}} \rightarrow \mathcal{C}_{\mathrm{S}}$. For clause (ii), it suffices to consider a chosen cartesian lifting $(f, \iota):\left(\Gamma, f^{*} B\right) \rightarrow(\Delta, B)$ of $\mathfrak{T}_{\mathrm{S}}$. Taking advantage of the 2-fully faithfulness of $E: \mathfrak{T}_{\mathrm{S}} \rightarrow \mathfrak{C}_{\mathrm{S}}^{\mathbf{2}}$, we may express the property 
we are to verify as follows: that for each diagram

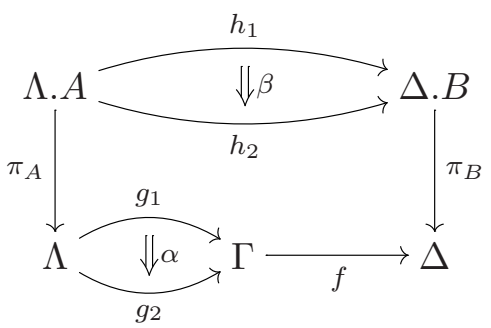

in $\mathfrak{C}_{\mathrm{S}}$ with $\pi_{B} \beta=f \alpha \pi_{A}$, there is a unique factorisation

$$
\beta=\Lambda . A \underset{h_{2}^{\prime}}{\stackrel{h_{1}^{\prime}}{\Downarrow_{\beta^{\prime}}}} \Gamma \cdot f^{*} B \stackrel{f . \iota}{\longrightarrow} \Delta . B
$$

with $\pi_{f^{*} B} \beta^{\prime}=\beta \pi_{A}$. But this follows without difficulty from the fact that diagram (17) is a 2-pullback. For clause (iii) in the definition of 2-fibration, we suppose given $(\Gamma, A)$ and $(\Delta, B)$ in $\mathfrak{T}_{\mathrm{S}}$ and are required to show that the functor $\mathfrak{T}_{\mathrm{S}}((\Gamma, A),(\Delta, B)) \rightarrow \mathfrak{C}_{\mathrm{S}}(\Gamma, \Delta)$ is a fibration. Using once more the 2-fully faithfulness of $E$, it suffices to show that for each commutative square

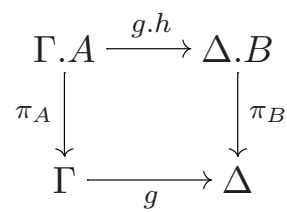

in $\mathfrak{C}_{\mathrm{S}}$ and 2-cell $\alpha: f \Rightarrow g$, we can find a 1-cell $k: \Gamma . A \rightarrow \Delta . B$ and a 2-cell $\beta: k \Rightarrow g . h$ satisfying $\pi_{B} k=f \pi_{A}$ and $\pi_{B} \beta=\alpha \pi_{A}$. This follows using the canonical isofibration structure of $\pi_{B}$. Finally, for clause (iv), we must show that each $(-) \cdot f: \mathfrak{T}_{\mathrm{S}}(y, z) \rightarrow \mathfrak{T}_{\mathrm{S}}(x, z)$ preserves cartesian liftings of 2-cells. As every 2-cell of $\mathfrak{T}_{\mathrm{S}}$ is invertible, and hence cartesian, this is automatic.

We end this section by considering the pullback stability of the canonical isofibration structures of Proposition 3.4.7. To this end, consider a square like (17). Both vertical arrows $\pi_{B}$ and $\pi_{f^{*} B}$ have their canonical isofibration structures; but we also have a second isofibration structure on $\pi_{f^{*} B}$, obtained by pulling back the canonical structure of $\pi_{B}$ along $f$. A careful examination of the proof of Proposition 3.4.7 reveals that these two structures on $\pi_{f^{*} B}$ need not coincide. In other words, the canonical isofibration structures of Proposition 3.4.7 are not necessarily stable by pullbacks. At first glance, this may appear surprising, since stability by pullbacks tends to be an automatic consequence of stability under substitution. However, a more careful analysis shows that in this case, stability under substitution corresponds to a more restricted form of pullback stability, which we now describe. 
Suppose we are given $\Delta \in \mathfrak{C}_{\mathrm{S}}, A \in \mathfrak{T}_{\mathrm{S}}(\Delta)$ and $B \in \mathfrak{T}_{\mathrm{S}}(\Delta . A)$. We can view the dependent projection $\pi_{B}: \Delta . A . B \rightarrow \Delta$.A not only as a map of $\mathfrak{C}_{\mathrm{S}}$, but also as a map

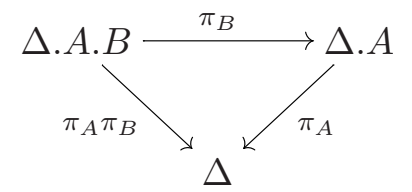

of $\mathfrak{C}_{\mathrm{S}} / \Delta$. It is easy to see that the forgetful 2-functor $\mathfrak{C}_{\mathrm{S}} / \Delta \rightarrow \mathfrak{C}_{\mathrm{S}}$ creates normal isofibrations, so that $(26)$ is canonically a normal isofibration in $\mathfrak{C}_{\mathrm{S}} / \Delta$. Suppose we are now given a morphism $f: \Gamma \rightarrow \Delta$ of $\mathfrak{C}_{\mathrm{S}}$. By pulling back (26) along $f$, we obtain the map

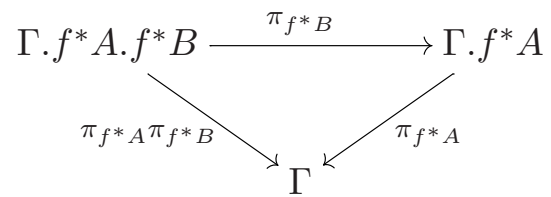

of $\mathfrak{C}_{\mathrm{S}} / \Gamma$ (note that we are abusing notation slightly: we should really write the left-hand vertex as $\left.\Gamma . f^{*} A \cdot(f . \iota)^{*} B\right)$, and this now has two isofibration structures on it: the one induced by the canonical isofibration structure on $\pi_{f^{*} B}$, and the one obtained by pulling back the isofibration structure of (26). The following Proposition now tells us that these two isofibration structures on (27) do coincide.

Proposition 3.4.10. Suppose given $\Delta \in \mathfrak{C}_{\mathrm{S}}, A \in \mathfrak{T}_{\mathrm{S}}(\Delta)$ and $B \in \mathfrak{T}_{\mathrm{S}}(\Delta . A)$ and $f: \Gamma \rightarrow$ $\Delta$ as above. With reference to the 2-pullback square

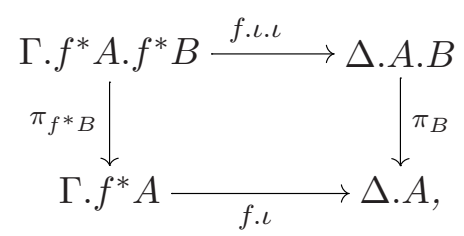

the canonical isofibration structure on $\pi_{f^{*} B}$ qua map of $\mathfrak{C}_{\mathrm{S}} / \Gamma$ agrees with the pullback along $f$ of the canonical isofibration structure on $\pi_{B}$ qua map of $\mathfrak{C}_{\mathrm{S}} / \Delta$.

Proof. As in the proof of Proposition 3.4.5, we will use subst notation in applications of the Leibniz rule, in order to make clear the dependent family in which substitution is taking place. Now, to prove the Proposition, it suffices to show the following. Suppose give an invertible 2 -cell

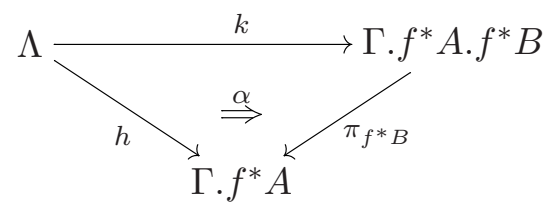

of $\mathfrak{C}_{\mathrm{S}} / \Gamma$ (i.e., one satisfying $\pi_{f^{*} A} \alpha=\mathrm{id}_{\pi_{f^{*} A} h}$ ). Let us write $\alpha^{\prime}:=f . \iota \circ \alpha$ and $k^{\prime}:=f . \iota . \iota \circ k$. Then we must show that

$$
s_{\alpha^{\prime}}=f . \iota . \iota \circ s_{\alpha}: \Lambda \rightarrow \Delta . A . B \quad \text { and } \quad \sigma_{\alpha^{\prime}}=f . \iota . \iota \circ \sigma_{\alpha}: s_{\alpha^{\prime}} \Rightarrow k^{\prime},
$$


where we obtain $\left(s_{\alpha}, \sigma_{\alpha}\right)$ from the canonical isofibration structure on $\pi_{f^{*} B}$, and $\left(s_{\alpha^{\prime}}, \sigma_{\alpha^{\prime}}\right)$ from that on $\pi_{B}$. So suppose given a 2-cell as in (29), with $h, k$ and $\alpha$ given as follows:

$$
\begin{array}{ll} 
& x: \Lambda \vdash h(x):=\left(h_{1} x, h_{2} x\right): \Gamma \cdot f^{*} A \\
& x: \Lambda \vdash k(x):=\left(h_{1} x, k_{2} x, k_{3} x\right): \Gamma \cdot f^{*} A \cdot f^{*} B \\
\text { and } \quad & x: \Lambda \vdash \alpha(x):=\left(\operatorname{rh}_{1} x, \alpha_{2} x\right): \operatorname{ld}_{\Gamma \cdot f^{*} A}\left(\left(h_{1} x, h_{2} x\right),\left(h_{1} x, k_{2} x\right)\right) .
\end{array}
$$

We first compute the pair $\left(s_{\alpha}, \sigma_{\alpha}\right)$. The map $s_{\alpha}: \Lambda \rightarrow \Gamma \cdot f^{*} A . f^{*} B$ is given by

$$
x: \Lambda \vdash\left(h_{1} x, h_{2} x, \operatorname{subst}_{[u, v] B(f u, v)}\left(\left(\mathrm{r} h_{1} x, \alpha_{2} x\right), k_{3} x\right)\right): \Gamma \cdot f^{*} A . f^{*} B ;
$$

which, by unfolding the inductive description of the Id-elimination rule given in the proof of Proposition 3.3.1, is equal to

$$
x: \Lambda \vdash\left(h_{1} x, h_{2} x, \operatorname{subst}_{[v] B\left(f h_{1} x, v\right)}\left(\alpha_{2} x, k_{3} x\right)\right): \Gamma \cdot f^{*} A . f^{*} B .
$$

The corresponding 2-cell $\sigma_{\alpha}: s_{\alpha} \Rightarrow k$ is now given by

$$
x: \Lambda \vdash\left(\mathrm{rh}_{1} x, \alpha_{2} x, \mathrm{r}\left(\operatorname{subst}_{[v] B\left(f h_{1} x, v\right)}\left(\alpha_{2} x, k_{3} x\right)\right)\right): \operatorname{ld}\left(s_{\alpha} x, k x\right) .
$$

Next we compute the pair $\left(s_{\alpha^{\prime}}, \sigma_{\alpha^{\prime}}\right)$. By the proof of Proposition 3.4.5 we have

$$
\begin{aligned}
\alpha^{\prime}(x) & :=(f . \iota)^{\bullet}\left(\mathrm{r}_{1} x, \alpha_{2} x\right) \\
& =\left(f \bullet r h_{1} x, \theta\left(\mathrm{r} h_{1} x, k_{2} x\right) \circ \alpha_{2} x\right) \\
& =\left(\mathrm{r} f h_{1} x, \mathrm{r}\left(k_{2} x\right) \circ \alpha_{2} x\right)=\left(\mathrm{r} f h_{1} x, \alpha_{2} x\right) .
\end{aligned}
$$

Thus the morphism $s_{\alpha^{\prime}}: \Gamma \rightarrow \Delta . A . B$ is given by

$$
x: \Lambda \vdash\left(f h_{1} x, h_{2} x, \operatorname{subst}_{[u, v] B(u, v)}\left(\left(\operatorname{r} f h_{1} x, \alpha_{2} x\right), k_{3} x\right)\right): \Delta . A . B ;
$$

which, by unfolding the description of Id-elimination, is definitionally equal to

$$
x: \Lambda \vdash\left(f h_{1} x, h_{2} x, \operatorname{subst}_{[v] B\left(f h_{1} x, v\right)}\left(\alpha_{2} x, k_{3} x\right)\right): \Delta . A . B .
$$

The corresponding 2-cell $\sigma_{\alpha^{\prime}}: s_{\alpha^{\prime}} \Rightarrow k^{\prime}$ is now given by

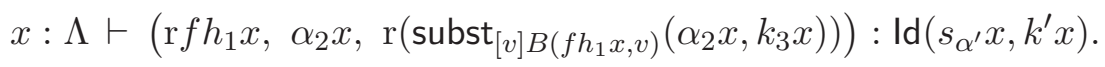

It remains to verify the equalities in (30). The first equality follows immediately from inspection of (31) and (33). For the second, we will need a calculation. Suppose given $(x, y, s)$ and $(x, z, t): \Lambda \cdot f^{*} A \cdot f^{*} B$ together with identity proofs $p: \operatorname{Id}(y, z)$ and $q: \operatorname{ld}\left(s, \operatorname{subst}_{[v] B(f x, v)}(p, t)\right)$. We claim that:

$$
(f . \iota . \iota)^{\bullet}(\mathrm{r}(x), p, q)=(\mathrm{r}(f x), p, q): \mathrm{ld}_{\Delta . A . B}((f x, y, s),(f x, z, t)) .
$$

By discrete identity types, it suffices to prove this up to propositional equality; and by applying Id-elimination twice, first on $p$ and then on $q$, it suffices for this to show that, given $(x, y, s): \Gamma \cdot f^{*} A . f^{*} B$, we have

$$
(f . \iota . \iota)^{\bullet}(\mathrm{r} x, \mathrm{r} y, \mathrm{r} k) \approx(\mathrm{r} f x, \mathrm{r} y, \mathrm{r} k): \mathrm{ld}_{\Delta . A . B}((f x, y, s),(f x, y, s)) .
$$

But this follows by the Id-computation rule and the definition of $(f . \iota . \iota)^{\bullet}$. Thus we have (35) as claimed. We now use this to affirm the second equality in (30). Given $x: \Lambda$, we have that:

$$
\begin{aligned}
\left(f . \iota . \iota \circ \sigma_{\alpha}\right)(x) & =(f . \iota . \iota)^{\bullet}\left(\mathrm{rh}_{1} x, \alpha_{2} x, \mathrm{r}_{\left.\left(\operatorname{subst}_{[v] B\left(f h_{1} x, v\right)}\left(\alpha_{2} x, k_{3} x\right)\right)\right)}\right. \\
& =\left(\mathrm{r} f h_{1} x, \alpha_{2} x, \mathrm{r}\left(\text { subst }_{[v] B\left(f h_{1} x, v\right)}\left(\alpha_{2} x, k_{3} x\right)\right)\right) \\
& =\sigma_{f . \iota \circ \alpha}(x)=\sigma_{\alpha^{\prime}}(x) .
\end{aligned}
$$


Remark 3.4.11. Although it may seem somewhat technical, the result we have just proven is absolutely crucial for obtaining a sound notion of two-dimensional model. Without it, our models would not necessarily be sound for the rules expressing stability of the elimination rules under change of ambient context. It is not just the identity type rules would be afflicted either: we will see in $\$ 5.2-\$ 5.5$ below that Proposition 3.4.10 is used in verifying the stability under substitution of all the type-theoretic elimination rules. One of the key issues in giving higher-dimensional and homotopy-theoretic semantics for intensional type theory will be finding an appropriate counterpart of this Proposition.

3.5. Comprehension 2-categories. We may abstract away from the syntactic investigations of the preceding sections as follows. We define a full split comprehension 2category $\mathbb{C}$ to be given by the following data: a locally groupoidal 2-category $\mathfrak{C}$ with a specified 2-terminal object; a globally split 2-fibration $p: \mathfrak{T} \rightarrow \mathfrak{C}$, with $\mathfrak{T}$ also locally groupoidal; and a 2-fully faithful 2 -functor $E: \mathfrak{T} \rightarrow \mathfrak{C}^{2}$ rendering commutative the triangle

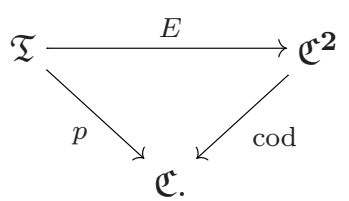

Moreover, the 2-functor $E$ should send cartesian morphisms in $\mathfrak{T}$ to 2-pullback squares in $\mathfrak{C}$; should send each object of $\mathfrak{T}$ to a normal isofibration in $\mathfrak{C}$; and should satisfy the stability conditions of Proposition 3.4.10. The preceding developments show that we may associate a full split comprehension 2-category to each dependent type theory $\mathrm{S}$ satisfying the rules for identity types in Table 1 and the discreteness rules of Table 2 . We denote this comprehension 2-category by $\mathbb{C}(\mathrm{S})$, and call it the classifying comprehension 2 -category of S.

\section{Categorical models For $\mathrm{ML}_{2}$ : LOGical aspects}

4.1. Identity types. In this section, we will examine the structure induced on the syntactic comprehension 2-category of the previous section by the logical rules of twodimensional type theory. Once again we consider a fixed dependently typed calculus $\mathrm{S}$ which we now suppose to admit all of the rules in Tables 1, 2 and 3. We begin by investigating the identity types. Given how deeply intertwined these have been with the construction of the syntactic comprehension 2-category, it is perhaps unsurprising that their characterisation is rather intrinsic. It will be given in terms of the 2-categorical notion of arrow object. Given a 2-category $\mathbf{K}$, an arrow object for $X \in \mathbf{K}$ is given by an object $Y \in \mathbf{K}$ such that 1-cells into $Y$ correspond naturally to 2-cells into $X$. That is, we have an isomorphism of categories

$$
\mathbf{K}(A, Y) \cong \mathbf{K}(A, X)^{2},
$$

2-natural in $A$. In particular, under the bijection (36), the identity map $\operatorname{id}_{Y}: Y \rightarrow Y$ corresponds to a 2-cell

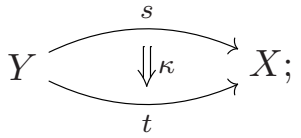


and 2-naturality of (36) says that any other such 2-cell into $X$ factors uniquely through $\kappa$. In the language of enriched category theory [21], an arrow object is a certain kind of (weighted) limit, namely a power (or sometimes cotensor) with the category 2. For a full treatment of 2-categorical limits, we refer the reader to [22].

We now introduce a small abuse of notation. Given $\Gamma \in \mathfrak{C}_{\mathrm{S}}$ and $A \in \mathfrak{T}_{\mathrm{S}}(\Gamma)$, we write $\Gamma . A$. $A$ for the context $(x: \Gamma, y: A(x), z: A(x))$-this rather than the more correct $\Gamma . A . \pi_{A}^{*} A$-and write $\pi_{1}$ and $\pi_{2}$ for the context morphisms $\Gamma . A . A \rightarrow \Gamma$.A projecting onto the first or second copy of $A$.

Proposition 4.1.1. For every context $\Gamma$ and type $\Gamma \vdash A$ type in $\mathrm{S}$, the context $\Gamma$.A.A.ld $A$, together with the projections $\pi_{1} \pi_{\mathrm{Id}_{A}}, \pi_{2} \pi_{\mathrm{Id}_{A}}: \Gamma$.A.A.ld $A \rightarrow \Gamma . A$, can be made into an arrow object for $\Gamma$.A in the slice 2 -category $\mathfrak{C}_{\mathrm{S}} / \Gamma$.

Proof. Let us write $s:=\pi_{1} \pi_{\mathrm{Id}_{A}}$ and $t:=\pi_{2} \pi_{\mathrm{Id}_{A}}$. We are to find a 2-cell

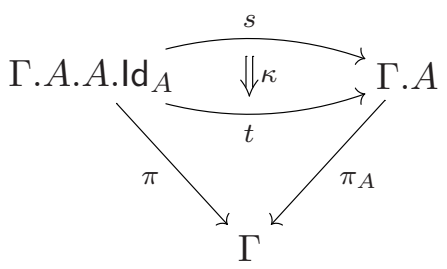

in $\mathfrak{C}_{\mathrm{S}}$ which is over $\Gamma$ in the sense that $\pi_{A} s=\pi_{A} t=\pi$ and $\pi_{A} \kappa=\mathrm{id}_{\pi}$, and such that any other 2-cell

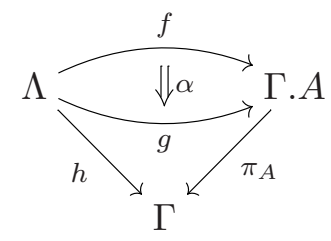

over $\Gamma$ factors through $\kappa$ via a unique morphism $\bar{\alpha}: \Lambda \rightarrow \Gamma \cdot A \cdot A \cdot \mathrm{Id}_{A}$. The universal property of $\kappa$ also has a two-dimensional aspect. Suppose we are given a commutative diagram

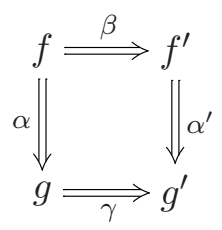

of 1- and 2-cells $\Lambda \rightarrow \Gamma$.A over $\Gamma$. Then we should be able to find a unique 2-cell $\delta: \bar{\alpha} \Rightarrow \bar{\alpha}^{\prime}: \Lambda \rightarrow \Gamma$.A.A.ld ${ }_{A}$ with $\beta=s \delta$ and $\gamma=t \delta$. We begin by defining $\kappa$ as in (37). For this we are required to give a judgement

$$
x: \Gamma, y, z: A(x), p: \operatorname{ld}(y, z) \vdash \kappa(x, y, z, p): \operatorname{ld}(y, z) ;
$$

which we do by taking $\kappa(x, y, z, p):=p$. We now verify the universal property of $\kappa$. Suppose given an $\alpha$ as in (38): then the commutativity conditions $\pi_{A} f=\pi_{A} g=h$ mean that $f$ and $g$ correspond to judgements

$$
x: \Lambda \vdash f(x): A(h x) \quad \text { and } \quad x: \Lambda \vdash g(x): A(h x),
$$


whereupon - by Lemma 3.4.2 - the condition $\pi_{A} \alpha=\mathrm{id}_{h}$ allows us to view $\alpha$ as a judgement $x: \Lambda \vdash \alpha(x): \operatorname{ld}(f x, g x)$. We now define a morphism $\bar{\alpha}: \Lambda \rightarrow \Gamma \cdot A \cdot A \cdot \operatorname{ld}_{A}$ by $x: \Lambda \vdash(h x, f x, g x, \alpha x): \Gamma . A . A . \mathrm{Id}_{A}$. It is immediate from the definition of $\kappa$ that $\kappa \bar{\alpha}=\alpha$, and moreover that if $\kappa m=\alpha$ for some $m: \Lambda \rightarrow \Gamma$.A.A. Id $_{A}$ then we have $\bar{\alpha}=m$. It still remains to verify the two-dimensional universal property of $\kappa$. So suppose given 1 - and 2-cells as in (39). We are required to define a 2-cell $\delta: \bar{\alpha} \Rightarrow \bar{\alpha}^{\prime}: \Lambda \rightarrow \Gamma$.A.A. Id $_{A}$ satisfying $s \delta=\beta$ and $t \delta=\gamma$. In order to satisfy these last two requirements, $\delta$, if it exists, must be given by a judgement

$$
x: \Lambda \vdash\left(\operatorname{rh} x, \beta x, \gamma x, \delta_{4} x\right): \operatorname{Id}_{\Gamma . A . A . I_{A}}\left((h x, f x, g x, \alpha x),\left(h x, f^{\prime} x, g^{\prime} x, \alpha^{\prime} x\right)\right)
$$

for some $x: \Lambda \vdash \delta_{4}(x): \operatorname{ld}_{\operatorname{ld}(f x, g x)}\left(\alpha x,(\beta x, \gamma x)^{*}\left(\alpha^{\prime} x\right)\right)$. By discrete identity types, this is only possible if in fact $\alpha(x)=(\beta x, \gamma x)^{*}\left(\alpha^{\prime} x\right)$, whereupon we can take $\delta_{4}(x)=\mathrm{r}(\alpha x)$. We claim that in fact $(\beta x, \gamma x)^{*}\left(\alpha^{\prime} x\right)=(\gamma x)^{-1} \circ\left(\alpha^{\prime} x \circ \beta x\right)$, so that we will be done if we can show that $\alpha(x)=(\gamma x)^{-1} \circ\left(\alpha^{\prime} x \circ \beta x\right)$ : and this follows from the equation $\gamma \alpha=\alpha^{\prime} \beta$ using the groupoid laws for $\mathrm{Id}_{A}$. It remains only to prove the claim, which follows from the more general result that

$$
\begin{aligned}
x: \Gamma, y, z, y^{\prime}, z^{\prime}: A(x), p: \operatorname{ld}\left(y, y^{\prime}\right), q: \operatorname{ld}\left(z, z^{\prime}\right), & s: \operatorname{ld}\left(y^{\prime}, z^{\prime}\right) \\
& \vdash(p, q)^{*}(s)=q^{-1} \circ(s \circ p): \operatorname{ld}(y, z) .
\end{aligned}
$$

By discrete identity types, it suffices to prove this up to propositional equality; and by Id-elimination on $p$ and $q$, it suffices to consider the case where $y=y^{\prime}, z=z^{\prime}, p=\mathrm{r}(y)$ and $q=\mathrm{r}(z)$, where we have that $(\mathrm{r}(y), \mathrm{r}(z))^{*}(s)=s=\mathrm{r}(z)^{-1} \circ(s \circ \mathrm{r}(y))$ as required.

Proposition 4.1.2 (Stability for identity types). Let $\Gamma, \Delta$ be contexts in $\mathrm{S}$, let $f: \Gamma \rightarrow \Delta$ be a context morphism, and let $x: \Delta \vdash B(x)$ type. Then the comparison morphism

$$
\Gamma . f^{*} B . f^{*} B .(f . \iota . \iota)^{*}\left(\operatorname{ld}_{B}\right) \rightarrow \Gamma . f^{*} B . f^{*} B . \operatorname{ld}_{f^{*} B}
$$

induced by the universal property of $\operatorname{ld}_{f^{*} B}$ is an identity.

Proof. Immediate from the stability of identity types under substitution.

4.2. Digression on 2-categorical adjoints. Our characterisation of the remaining type constructors of $\mathrm{ML}_{2}$ will be given in terms of weak 2-categorical adjoints. We therefore break off at this point in order to give a brief summary of the 2-categorical notions necessary for this characterisation. Let $\mathbf{K}$ be a 2-category. By a retract equivalence in $\mathbf{K}$, we mean a pair of objects $x, y \in \mathbf{K}$, a pair of morphisms $i: x \rightarrow y$ and $p: y \rightarrow x$ satisfying $p i=\mathrm{id}_{x}$, and an invertible 2-cell $\theta: \mathrm{id}_{y} \Rightarrow i p$ satisfying $\theta i=\mathrm{id}_{i}$ and $p \theta=\mathrm{id}_{p}$. In these circumstances, we may call $i$ an injective equivalence - with the understanding that the extra data $(p, \theta)$ is provided as part of this assertion - or call $p$ a surjective equivalence (with the same understanding). Given now a 2-functor $U: \mathbf{K} \rightarrow \mathbf{L}$ and an object $x \in \mathbf{L}$, we define a retract bireflection of $x$ along $U$ to be an object $F x \in \mathbf{K}$ and morphism $\eta_{x}: x \rightarrow U F x$ such that for each $y \in \mathbf{K}$, the functor

$$
\mathbf{K}(F x, y) \stackrel{U_{F x, y}}{\longrightarrow} \mathbf{L}(U F x, U y) \stackrel{(-) \circ \eta_{x}}{\longrightarrow} \mathbf{L}(x, U y)
$$

is a surjective equivalence of categories. By a left retract biadjoint $F$ for $U$, we mean a choice for every $x \in \mathbf{L}$ of a retract bireflection $F x$ of $x$ along $U$. Note that if $F$ is a left retract biadjoint for $U$, then the assignation $x \mapsto F x$ will not in general extend to a 2-functor $F: \mathbf{L} \rightarrow \mathbf{K}$; rather, it gives a pseudo-functor, which preserves identities 
and composition only up to invertible 2-cells. Likewise, the maps $\eta_{x}: x \rightarrow U F x$ do not provide components of a 2-natural transformation $\eta: \mathrm{id}_{\mathbf{L}} \Rightarrow U F$ but merely of a pseudonatural transformation, whose naturality squares commute only up to invertible 2-cells. We could give a definition of left retract biadjoint in terms of a pseudo-functor $\mathbf{K} \rightarrow \mathbf{L}$ and unit and counit transformations $\eta$ and $\epsilon$ satisfying weakened versions of the triangle laws (see [28, Section 1] for the details); but the above description is both simpler and, as we will see, closer to the type theory. In fact, the above definitions admit a further simplification, using the observation that the surjective equivalences of categories are precisely those functors $F: \mathcal{C} \rightarrow \mathcal{D}$ which are fully faithful and whose object function ob $F:$ ob $\mathcal{C} \rightarrow$ ob $\mathcal{D}$ is a split epimorphism:

Proposition 4.2.1. To give a retract bireflection of $x \in \mathbf{L}$ along $U: \mathbf{K} \rightarrow \mathbf{L}$ is to give an object $F x \in \mathbf{K}$ and map $\eta_{x}: x \rightarrow U F x$, together with, for each $f: x \rightarrow U y$ in $\mathbf{L}, a$ choice of map $\bar{f}: F x \rightarrow y$ in $\mathbf{K}$ satisfying $U \bar{f} \circ \eta_{x}=f$; all subject to the requirement that, for every $h, k: F x \rightarrow y$ in $\mathbf{K}$ and every $\alpha: U h \circ \eta_{x} \Rightarrow U k \circ \eta_{x}$ in $\mathbf{L}$, there is a unique $\bar{\alpha}: h \Rightarrow k$ with $U \bar{\alpha} \circ \eta_{x}=\alpha$.

Given a 2-functor $U: \mathbf{K} \rightarrow \mathbf{L}$ and $x \in \mathbf{L}$ as before, we have the dual notion of retract bicoreflection of $x$ along $U$ : this being given by an object $G x \in \mathbf{K}$, together with a morphism $\epsilon_{x}: U G x \rightarrow x$ such that for each $y \in \mathbf{K}$, the functor

$$
\mathbf{K}(y, G x) \stackrel{U_{y, G x}}{\longrightarrow} \mathbf{L}(U y, U G x) \stackrel{\epsilon_{x} \circ(-)}{\longrightarrow} \mathbf{L}(U y, x)
$$

is a surjective (not injective!) equivalence of categories. Now a right retract biadjoint for $U$ is of course given by a choice for every $x \in \mathbf{L}$ of a retract bicoreflection along $U$. As before, we have an elementary characterisation of retract bicoreflections:

Proposition 4.2.2. To give a retract bicoreflection of $x \in \mathbf{L}$ along $U: \mathbf{K} \rightarrow \mathbf{L}$ is to give an object $G x \in \mathbf{K}$ and map $\epsilon_{x}: U G x \rightarrow x$, together with, for each $f: U y \rightarrow x$ in $\mathbf{L}, a$ choice of map $\bar{f}: y \rightarrow G x$ in $\mathbf{K}$ satisfying $\epsilon_{x} \circ U \bar{f}=f$; all subject to the requirement that, for every $h, k: y \rightarrow G x$ in $\mathbf{K}$ and every $\alpha: \epsilon_{x} \circ U h \Rightarrow \epsilon_{x} \circ U k$ in $\mathbf{L}$, there is a unique $\bar{\alpha}: h \Rightarrow k$ with $\epsilon_{x} \circ U \bar{\alpha}=\alpha$.

4.3. Unit types. Our first application of the 2-categorical adjoint notions developed above will be to the unit types of $\mathrm{S}$ - which we recall is an arbitrary dependent type theory admitting all the rules listed in Tables 1, 2 and 3. In the following result, we denote by $E(\Gamma): \mathfrak{T}_{\mathrm{S}}(\Gamma) \rightarrow \mathfrak{C}_{\mathrm{S}} / \Gamma$ the 2-functor obtained by restricting $E: \mathfrak{T}_{\mathrm{S}} \rightarrow \mathfrak{C}_{\mathrm{S}}$ to the fibre over $\Gamma \in \mathfrak{C}_{\mathrm{S}}$.

Proposition 4.3.1. For each context $\Gamma$ of $\mathrm{S}$, the object $\mathbf{1}_{\Gamma} \in \mathfrak{T}_{\mathrm{S}}(\Gamma)$ given by $\Gamma \vdash \mathbf{1}$ type provides a retract bireflection of $\operatorname{id}_{\Gamma}: \Gamma \rightarrow \Gamma$ along the 2 -functor $E(\Gamma): \mathfrak{T}_{\mathrm{S}}(\Gamma) \rightarrow \mathfrak{C}_{\mathrm{S}} / \Gamma$.

Proof. The unit of the bireflection $\eta_{\Gamma}: \Gamma \rightarrow \Gamma . \mathbf{1}_{\Gamma}$ (over $\Gamma$ ) is given by the judgement $x: \Gamma \vdash \star: 1$. Given now a morphism $f: \Gamma \rightarrow \Gamma$.A over $\Gamma$-which is equally well a judgement $x: \Gamma \vdash f(x): A(x)$-we obtain a factorisation $\bar{f}: \Gamma \cdot \mathbf{1}_{\Gamma} \rightarrow \Gamma . A$ over $\Gamma$ by 1-elimination, taking $\bar{f}$ to be the term $x: \Gamma, z: \mathbf{1} \vdash \mathrm{U}_{f(x)}(z): A(x)$. That this satisies $\bar{f} \eta_{\Gamma}=f$ is now precisely the computation rule $x: \Gamma \vdash \mathrm{U}_{f(x)}(\star)=f(x)$. It remains to check that for maps $h, k: \Gamma .1 \rightarrow \Gamma$.A over $\Gamma$, every 2 -cell $\alpha: h \eta_{\Gamma} \Rightarrow k \eta_{\Gamma}$ over $\Gamma$ is of the 
form $\bar{\alpha} \eta_{\Gamma}$ for a unique $\bar{\alpha}: h \Rightarrow k$. Now, to give $h, k$ and $\alpha$ is to give judgements

$$
\begin{aligned}
x: \Gamma, z: \mathbf{1} & \vdash h(x, z): A(x) \\
x: \Gamma, z: \mathbf{1} & \vdash k(x, z): A(x) \\
x & : \Gamma \vdash \alpha(x): \operatorname{ld}(h(x, \star), k(x, \star)) ;
\end{aligned}
$$

from which we must determine $x: \Gamma, z: \mathbf{1} \vdash \bar{\alpha}(x, z): \operatorname{ld}(h(x, z), k(x, z))$. We do this by 1-elimination, taking $\bar{\alpha}(x, z):=\mathrm{U}_{\alpha(x)}(z)$. The equality $\bar{\alpha} \eta_{\Gamma}=\alpha$ now follows from the 1-computation rule. It remains to check uniqueness of $\bar{\alpha}$. So suppose we are given $x: \Gamma, z: \mathbf{1} \vdash \beta(x, z): \operatorname{ld}(h(x, z), k(x, z))$ satisfying $\beta(x, \star)=\alpha(x)$. We must show that $\beta(x, z)=\bar{\alpha}(x, z)$. By discrete identity types, it suffices to show this up to propositional equality; and by 1-elimination, this only in the case where $z=\star$, for which we have that $\beta(x, \star)=\alpha(x)=\bar{\alpha}(x, \star)$ as required.

Proposition 4.3.2 (Stability for unit types). For each $k: \Gamma \rightarrow \Delta$ in $\mathfrak{C}$, we have $k^{*}\left(\mathbf{1}_{\Delta}\right)=$ $\mathbf{1}_{\Gamma}$; we have $\eta_{\Gamma}=k^{*}\left(\eta_{\Delta}\right): \Gamma \rightarrow \Gamma . \mathbf{1}_{\Gamma}$; and for each $f: \Delta \rightarrow \Delta$.B over $\Delta$, have $k^{*}(\bar{f})=$ $\overline{k^{*}(f)}: \Gamma . \mathbf{1}_{\Gamma} \rightarrow \Gamma . k^{*} B$.

Proof. By the stability of unit types under substitution.

Remark 4.3.3. Note carefully what the previous result does not say: it does not say that for a context morphism $k: \Gamma \rightarrow \Delta$, the comparison map $\mathbf{1}_{\Gamma} \rightarrow k^{*} \mathbf{1}_{\Delta}$ of $\mathfrak{T}_{\mathrm{S}}(\Gamma)$ is an identity; indeed, this map will in general only be isomorphic to the identity, since it corresponds to the judgement $x: \Gamma, z: \mathbf{1} \vdash \mathrm{U}_{\star}(z): \mathbf{1}$.

4.4. Dependent sum types. We next consider the dependent sum types.

Proposition 4.4.1. For each context $\Gamma$ and type $\Gamma \vdash A$ type of $\mathrm{S}$, the 2-functor $\Delta_{A}:=$ $\mathfrak{T}_{\mathrm{S}}\left(\pi_{A}\right): \mathfrak{T}_{\mathrm{S}}(\Gamma) \rightarrow \mathfrak{T}_{\mathrm{S}}(\Gamma . A)$ has a left retract biadjoint $\Sigma_{A}$.

Proof. We must provide, for each $B \in \mathfrak{T}_{\mathrm{S}}(\Gamma . A)$ a retract bireflection $\Sigma_{A}(B)$ of $B$ along $\Delta_{A}$. So we take $\Sigma_{A}(B) \in \mathfrak{T}_{\mathrm{S}}(\Gamma)$ to be given by the judgement $\Gamma \vdash \Sigma(A, B)$ type (where for readability we suppress explicit mention of dependencies on the variables in $\Gamma$ ); and the unit map $\eta: B \rightarrow \Delta_{A} \Sigma_{A}(B)$ of $\mathfrak{T}_{\mathrm{S}}(\Gamma . A)$ to be given by the judgement $\Gamma, y: A, z:$ $B(y) \vdash\langle y, z\rangle: \Sigma(A, B)$. Now given a type $C \in \mathfrak{T}_{\mathrm{S}}(\Gamma)$ and a map $f: B \rightarrow \Delta_{A} C$ of $\mathfrak{T}_{\mathrm{S}}(\Gamma . A)$, we must provide a morphism $\bar{f}: \Sigma_{A}(B) \rightarrow C$ of $\mathfrak{T}_{\mathrm{S}}(\mathcal{C})$ satisfying $\Delta_{A}(\bar{f}) \circ \eta=f$. But to give $f$ is to give a judgement $\Gamma, y: A, z: B(y) \vdash f(y, z): C$, whilst to give $\bar{f}$ is to give a judgement $\Gamma, s: \Sigma(A, B) \vdash \bar{f}(s): C$. Thus by using $\Sigma$-elimination we may define $\bar{f}(s):=\mathrm{E}_{f}(s)$. The equality $\Delta_{A}(\bar{f}) \circ \eta=f$ follows by the $\Sigma$-computation rule. It remains to show, given two morphisms $h, k: \Sigma_{A}(B) \rightarrow D$ in $\mathfrak{T}_{\mathrm{S}}(\Gamma)$, that each 2-cell $\alpha: \Delta_{A}(h) \circ \eta \Rightarrow \Delta_{A}(k) \circ \eta$ is of the form $\Delta_{A}(\bar{\alpha}) \circ \eta$ for a unique $\bar{\alpha}: h \Rightarrow k$. This follows by an argument analogous to that given in the proof of Proposition 4.3.1.

Whilst Proposition 4.4.1 is very natural from a categorical perspective, it fails to capture the full strength of the elimination rule for dependent sums (even though it requires the full strength of that elimination rule in its proof). In order to do this, we need the following result: 
Proposition 4.4.2. Suppose given a context $\Gamma$ in $\mathrm{S}$ and types $\Gamma \vdash A$ type and $\Gamma, x$ : $A \vdash B(x)$ type in $\mathrm{S}$, and consider the morphism

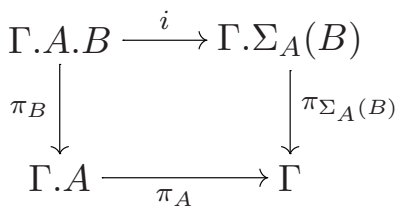

in $\mathfrak{C}_{\mathrm{S}}^{2}$ corresponding to the unit morphism $\eta: B \rightarrow \Delta_{A} \Sigma_{A}(B)$ in $\mathfrak{T}_{\mathrm{S}}(\Gamma . A)$. The map $i$ appearing in this diagram is an injective equivalence in $\mathfrak{C}_{\mathrm{S}} / \Gamma$.

Proof. We construct a pseudoinverse retraction for $i$ over $\Gamma$ as follows. The map $p: \Gamma \cdot \Sigma_{A}(B) \rightarrow$ $\Gamma . A . B$ over $\Gamma$ is given by the projections out of the sum:

$$
\begin{aligned}
& \Gamma, s: \Sigma(A, B) \vdash s .1: A \\
& \Gamma, s: \Sigma(A, B) \vdash s .2: B(s .1)
\end{aligned}
$$

(where again, we suppress explicit mention of the dependency on $\Gamma$ ). We define these by $\Sigma$-elimination on $s$, the first being given by $s .1:=\mathrm{E}_{[y, z] y}(s)$ and the second by $s .2:=$ $\mathrm{E}_{[y, z] z}(s)$. The equality $p i=\mathrm{id}_{\Gamma \cdot A . B}$ follows from the $\Sigma$-computation rule. We must now give a 2-cell $\theta: \operatorname{id}_{\Gamma \cdot \Sigma_{A}(B)} \Rightarrow i p$; which is equally well a judgement $\Gamma, s: \Sigma(A, B) \vdash \theta(s)$ : $\operatorname{Id}(s,\langle s .1, s .2\rangle)$. By $\Sigma$-elimination on $s$, it suffices to define $\theta$ when $s=\langle y, z\rangle$; whereupon we have $\langle s .1, s .2\rangle=\langle\langle y, z\rangle .1,\langle y, z\rangle .2\rangle=\langle y, z\rangle$ so that we can take $\theta(\langle y, z\rangle)=\mathrm{r}(\langle y, z\rangle)$. The equality $\theta i=\mathrm{id}_{i}$ now follows by the $\Sigma$-computation rule; and it remains only to verify that $p \theta=\mathrm{id}_{p}$. Now, $p \theta$ corresponds to the judgement

$$
\Gamma, s: \Sigma(A, B) \vdash p^{\bullet}(\theta(s)): \operatorname{ld}_{\Gamma . A . B}((s .1, s .2),(s .1, s .2)) \text {; }
$$

and we must show that in fact $p^{\bullet}(\theta(s))=\mathrm{r}(p(s))$. By discrete identity types, it suffices to show this up to propositional equality; and by $\Sigma$-elimination, this only when $s=\langle y, z\rangle$. But we calculate that $p^{\bullet}(\theta(\langle y, z\rangle))=p^{\bullet}(\mathrm{r}(\langle y, z\rangle))=\mathrm{r}(p(\langle y, z\rangle))$ as required.

Proposition 4.4.3 (Stability for dependent sums). Given $k: \Gamma \rightarrow \Lambda$ in $\mathfrak{C}_{\mathrm{S}}, A \in \mathfrak{T}(\Lambda)$ and $B \in \mathfrak{T}(\Lambda . A)$, we have that $k^{*}\left(\Sigma_{A}(B)\right)=\Sigma_{k^{*} A}\left(k^{*} B\right)$; that $k^{*}\left(\eta_{A, B}\right)=\eta_{k^{*} A, k^{*} B}$; and for each $f: B \rightarrow \Delta_{A} C$ in $\mathfrak{T}_{\mathrm{S}}(\Lambda . A)$, that $k^{*} \bar{f}=\overline{k^{*} f}: \Sigma_{k^{*} A}\left(k^{*} B\right) \rightarrow k^{*} C$. Moreover, reindexing along $k$ sends the injective equivalence structure on $i_{A, B}$ to the injective equivalence structure on $i_{k^{*} A, k^{*} B}$.

Proof. By the stability of dependent sum types under substitution.

4.5. Dependent product types. Finally, we turn to the categorical characterisation of dependent product types in S.

Proposition 4.5.1. For each context $\Gamma$ and type $\Gamma \vdash A$ type of $\mathrm{S}$, the weakening 2functor $\Delta_{A}: \mathfrak{T}_{\mathrm{S}}(\Gamma) \rightarrow \mathfrak{T}_{\mathrm{S}}(\Gamma . A)$ has a right retract biadjoint $\Pi_{A}$.

Proof. Once again, we suppress explicit mention of dependencies on the variables in $\Gamma$. We must provide, for each $B \in \mathfrak{T}_{\mathrm{S}}(\Gamma . A)$ a retract bicoreflection $\Pi_{A}(B)$ of $B$ along $\Delta_{A}$. For this we take $\Pi_{A}(B) \in \mathfrak{T}_{\mathrm{S}}(\Gamma)$ to be given by the judgement $\Gamma \vdash \Pi(A, B)$ type; and the counit map $\epsilon: \Delta_{A} \Pi_{A}(B) \rightarrow B$ of $\mathfrak{T}_{\mathrm{S}}(\Gamma \cdot A)$ to be given by the judgement $\Gamma, m:$ $\Pi(A, B), y: A \vdash m \cdot y: B(y)$. Now given a type $C \in \mathfrak{T}_{\mathrm{S}}(\Gamma)$ and a map $f: \Delta_{A} C \rightarrow B$ of $\mathfrak{T}_{\mathrm{S}}(\Gamma \cdot A)$, we are required to provide a morphism $\bar{f}: C \rightarrow \Pi_{A}(B)$ of $\mathfrak{T}_{\mathrm{S}}(\mathcal{C})$ satisfying 
$\epsilon \circ \Delta_{A}(\bar{f})=f$. So if $f$ is the judgement $\Gamma, y: C, z: A \vdash f(y, z): B(y)$, we take $\bar{f}$ to be the judgement $\Gamma, y: C \vdash \lambda z . f(y, z): \Pi(A, B)$. The equality $\epsilon \circ \Delta_{A}(\bar{f})=f$ follows by the $\beta$-rule.

It remains to show, given two morphisms $h, k: D \rightarrow \Pi_{A}(B)$ in $\mathfrak{T}_{\mathrm{S}}(\Gamma)$, that each 2-cell $\alpha: \epsilon \circ \Delta_{A}(h) \Rightarrow \epsilon \circ \Delta_{A}(k)$ can be written in the form $\epsilon \circ \Delta_{A}(\bar{\alpha})$ for a unique $\bar{\alpha}: h \Rightarrow k$. It is here that we will make crucial use of function extensionality. So, to give $h, k$ and $\alpha$ is to give judgements $\Gamma, C \vdash h: \Pi(A, B) ; \Gamma, C \vdash k: \Pi(A, B)$; and $\Gamma, C, z: A \vdash \alpha(z): \operatorname{ld}(h \cdot z, k \cdot z)$ (where we now suppress explicit mention of the dependency on $C$ ) and so we may define the 2-cell $\bar{\alpha}: h \Rightarrow k$ by applying the rule $\Pi$-ExT of Table 3 to obtain the judgement $\Gamma, C \vdash \operatorname{ext}(h, k, \alpha): \operatorname{ld}(h, k)$. We must now check that $\epsilon \circ \Delta_{A}(\bar{\alpha})=\alpha$. Recall from $\S 2.3$ the operation

$$
\frac{m, n: \Pi(A, B) \quad p: \operatorname{ld}(m, n) \quad a: A}{p * a: \operatorname{ld}(m \cdot a, n \cdot a)}
$$

given by $p * a:=\mathrm{J}_{[x] \mathrm{r}(x \cdot a)}(m, n, p)$. It is easy to see that $*$ is just the lifting of $\epsilon$ to identity types; so that $\epsilon \circ \Delta_{A}(\bar{\alpha})$ corresponds to the judgement

$$
\Gamma, C, z: A \vdash \operatorname{ext}(h, k, \alpha) * z: \operatorname{ld}(h \cdot z, k \cdot z) .
$$

But by the rule $\Pi$-EXT-APP of Table 3 , we have that $\operatorname{ext}(h, k, \alpha) * z=\alpha(z)$ as required. It remains to check uniqueness of $\bar{\alpha}$. So suppose that we are given $\Gamma, C \vdash \beta: \operatorname{ld}(h, k)$ satisfying $\beta * z=\alpha(z)$ : we must show that $\beta=\bar{\alpha}$. Now, because $\beta * z=\alpha(z)=\bar{\alpha} * z$, we have that

$$
\Gamma, C, z: A \vdash \operatorname{ext}(h, k,[z] \beta * z)=\operatorname{ext}(h, k,[z] \bar{\alpha} * z): \operatorname{ld}(h, k) .
$$

Thus we will be done if we can show that

$$
\Gamma, C, m, n: \Pi(A, B), k: \operatorname{ld}(m, n) \vdash \operatorname{ext}(m, n,[z] k * z)=k: \operatorname{ld}(m, n)
$$

holds. By discrete identity types, it suffices to do this up to propositional equality; and by Id-elimination, this only in the case where $m=n$ and $k=\mathrm{r}(m)$, so that we will be done if we can show that

$$
\Gamma, C, m: \Pi(A, B) \vdash \operatorname{ext}(m, m,[z] \mathrm{r}(m \cdot z)) \approx \mathrm{r}(m): \operatorname{ld}(m, m)
$$

holds. But this follows immediately from the rule П-EXT-COMP.

Proposition 4.5.2 (Stability for dependent products). Given $k: \Gamma \rightarrow \Lambda$ in $\mathfrak{C}_{\mathrm{S}}, A \in \mathfrak{T}(\Lambda)$ and $B \in \mathfrak{T}(\Lambda . A)$, we have that $k^{*}\left(\Pi_{A}(B)\right)=\Pi_{k^{*} A}\left(k^{*} B\right)$; that $k^{*}\left(\epsilon_{A, B}\right)=\epsilon_{k^{*} A, k^{*} B}$; and for each $f: \Delta_{A} C \rightarrow B$ in $\mathfrak{T}_{\mathrm{S}}(\Lambda . A)$, that $k^{*} \bar{f}=\overline{k^{*} f}: k^{*} C \rightarrow \Pi_{k^{*} A}\left(k^{*} B\right)$.

Proof. By the stability of dependent product types under substitution.

4.6. Models of two-dimensional type theory. We now abstract away from the preceding results as follows.

Definition 4.6.1. Let there be given a full split comprehension 2-category $\mathbb{C}=(p: \mathfrak{T} \rightarrow$ $\left.\mathfrak{C}, E: \mathfrak{C} \rightarrow \mathfrak{T}^{\mathbf{2}}\right)$, in the sense of $§ 3.5$. Then:

- We say that $\mathbb{C}$ has equality if, for every $\Gamma \in \mathfrak{C}$ and $A \in \mathfrak{T}(\Gamma)$, there is an object $\operatorname{ld}_{A} \in \mathfrak{T}(\Gamma . A . A)$ such that $\Gamma . A . A . \mathrm{Id}_{A}$, together with its two projections onto $\Gamma . A$, underlies an arrow object for $\Gamma . A$ in $\mathfrak{C} / \Gamma$; and these arrow objects satisfy the stability properties of Proposition 4.1.2. 
- We say that $\mathbb{C}$ has units if, for every $\Gamma \in \mathfrak{C}$, the map $\operatorname{id}_{\Gamma}: \Gamma \rightarrow \Gamma$ admits a retract bireflection $\mathbf{1}_{\Gamma}$ along $E(\Gamma): \mathfrak{T}(\Gamma) \rightarrow \mathfrak{C}_{\mathrm{S}} / \Gamma$; and these bireflections satisfy the stability properties of Proposition 4.3.2.

- We say that $\mathbb{C}$ has sums if, for every $\Gamma \in \mathfrak{C}$ and $A \in \mathfrak{T}(\Gamma)$, the 2-functor $\Delta_{A}:=\mathfrak{T}\left(\pi_{A}\right): \mathfrak{T}(\Gamma) \rightarrow \mathfrak{T}(\Gamma \cdot A)$ admits a retract left biadjoint $\Sigma_{A}$; and these biadjoints satisfy the conditions of Proposition 4.4.2 and the stability properties of Proposition 4.4.3.

- We say that $\mathbb{C}$ has products if, for every $\Gamma \in \mathfrak{C}$ and $A \in \mathfrak{T}(\Gamma)$, the 2-functor $\Delta_{A}: \mathfrak{T}(\Gamma) \rightarrow \mathfrak{T}(\Gamma . A)$ admits a retract right biadjoint $\Pi_{A}$; and these biadjoints satisfy the stability properties of Proposition 4.5.2.

- We say that $\mathbb{C}$ is a model of two-dimensional type theory if it has equality, units, sums and products.

Thus, the results of this section can be summarised by saying that, for any dependent type theory $\mathrm{S}$ satisfying the rules of Tables 1,2 and 3, the classifying comprehension 2-category $\mathbb{C}(\mathrm{S})$ is a model of two-dimensional type theory. With an eye on future applications, we end this Section by gathering together in one place a list of the structure required for a two-dimensional model of type theory.

Definition 4.6.2. A two-dimensional model of type theory $\mathbb{C}$ is given by:

- A locally groupoidal 2-category $\mathfrak{C}$ of contexts, with a specified 2-terminal object;

- A locally groupoidal 2-category $\mathfrak{T}$ of types-in-context;

- A globally split 2-fibration $p: \mathfrak{T} \rightarrow \mathfrak{C}$ in the sense of Definition 3.4.1. Spelling this out, this means that $p$ is a 2 -functor such that:

(i) The underlying ordinary functor of $p$ is a split fibration of categories;

(ii) For every cartesian 1-cell $f: y \rightarrow z$ of $\mathfrak{C}$ and every 2-cell $\alpha: g \Rightarrow h: x \rightarrow z$ of $\mathfrak{T}$, any factorisation of $p(\alpha)$ through $p(f)$ may be lifted uniquely to a factorisation of $\alpha$ through $f$.

(iii) For each $x, y \in \mathfrak{T}$, the induced functor $p_{x, y}: \mathfrak{T}(x, y) \rightarrow \mathfrak{C}(p x, p y)$ is a fibration of groupoids.

(Note that condition (iv) of Definition 3.4.1 is automatically satisfied since every fibre category is a groupoid).

- A comprehension 2-functor $E: \mathfrak{T} \rightarrow \mathfrak{C}^{2}$, equipped with:

(i) For each object $A \in \mathfrak{T}$, a normal isofibration structure on $E(A)$ in the sense of Definition 3.4.6.

and satisfying the following properties:

(i) $\operatorname{cod} \circ E=p$;

(ii) $E$ is 2-fully faithful (i.e., an isomorphism on hom-groupoids);

(iii) $E$ sends cartesian morphisms of $\mathfrak{T}$ to 2 -pullback squares in $\mathfrak{C}$;

(iv) The normal isofibration structures picked out by $E$ have the stability properties of Proposition 3.4.10.

In describing the remaining, logical, structure, we use freely the conventions of Notation 3.1.1.

- For every $\Gamma \in \mathfrak{C}$ and $A \in \mathfrak{T}(\Gamma)$, there is given an object $\operatorname{ld}_{A} \in \mathfrak{T}(\Gamma . A . A)$ and a 2-cell $\kappa: \pi_{1} \Rightarrow \pi_{2}: \Gamma$.A.A.ld $A \rightarrow \Gamma$.A over $\Gamma$ which together provide an arrow object (in the sense of $\S 4.1$ ) for $\Gamma . A$ in $\mathfrak{C} / \Gamma$.

- For every $\Gamma \in \mathfrak{C}$, there is given a retract bireflection (in the sense of Proposition 4.2.1) $\mathbf{1}_{A}$ of the object $\operatorname{id}_{\Gamma}: \Gamma \rightarrow \Gamma$ along $E(\Gamma): \mathfrak{T}(\Gamma) \rightarrow \mathfrak{C}_{S} / \Gamma$. 
- For every $\Gamma \in \mathfrak{C}$ and $A \in \mathfrak{T}(\Gamma)$, there are given both left and right retract biadjoints (in the sense of $\S 4.2) \Sigma_{A}$ and $\Pi_{A}$ for $\mathfrak{T}\left(\pi_{A}\right): \mathfrak{T}(\Gamma) \rightarrow \mathfrak{T}(\Gamma . A)$.

- For every $\Gamma \in \mathfrak{C}, A \in \mathfrak{T}(\Gamma)$ and $B \in \mathfrak{T}(\Gamma . A)$, there is given a choice of injective equivalence structure on the canonical morphism $i: \Gamma \cdot A \cdot B \rightarrow \Gamma \cdot \Sigma_{A} B$ defined as in (40).

- The above structures satisfy the stability properties listed in Propositions 4.1.2, 4.3.2, 4.4.3 and 4.5.2.

\section{The internal language of a two-Dimensional model}

5.1. 2-categorical lifting properties. In this Section, we prove a converse to the results of the previous two Sections. Given a model $\mathbb{C}$ of two-dimensional type theory, we will construct from it a dependent type theory $\mathrm{S}(\mathbb{C})$ admitting the rules of Tables 1,2 and 3 . We call this type theory the internal language of $\mathbb{C}$. The key to doing this will be to give semantic analogues in $\mathbb{C}$ of each of the logical rules of $\mathrm{ML}_{2}$. In giving analogues of the elimination rules, we will make use of the 2-categorical lifting property described in Proposition 5.1.1 below. This is again very much in the spirit of [2], since this is fundamentally a result about the weak factorisation system (injective equivalences, normal isofibrations) described in Remark 3.4.8: or rather, about an algebraic presentation of this weak factorisation system in the style of [12].

Proposition 5.1.1. Suppose given a 2-category $\mathbf{K}$ and a square

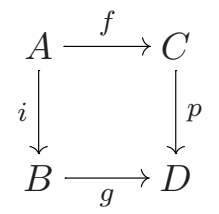

where $i$ carries the structure of an injective equivalence (cf. §4.2) and $p$ that of a normal isofibration (cf. Definition 3.4.6). From this data we can determine a canonical diagonal filler $j: B \rightarrow C$ satisfying $p j=g$ and $j i=f$.

Proof. The injective equivalence structure on $i$ is given by a morphism $k: B \rightarrow A$ satisfying $k i=\mathrm{id}_{A}$ and an invertible 2-cell $\theta: \operatorname{id}_{B} \Rightarrow i k$ satisfying $\theta i=\mathrm{id}_{i}$ and $k \theta=\mathrm{id}_{k}$. Thus we have an invertible 2-cell

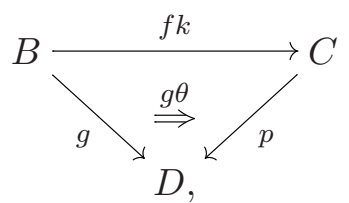

and so from the isofibration structure on $p$ we obtain a map $j:=s_{g \theta}: B \rightarrow C$ satisfying $p j=g$. It remains to show that $j i=f$. By the definition of isofibration, we have $j i=s_{g \theta} \circ i=s_{g \theta i}$; and since $s_{g \theta i}=s_{g\left(\mathrm{id}_{i}\right)}=s_{\mathrm{id}_{g i}}=s_{\mathrm{id}_{p f}}$, we deduce by normality that $j i=s_{\mathrm{id}_{p f}}=f$ as required.

We now show that the liftings of the previous Proposition are stable under pullback in a suitable sense. Note that in order for this to make sense, it is crucial that Proposition 5.1.1 gives us a choice of filler for each diagram like (41). 
Proposition 5.1.2. Suppose given a morphism $h: X \rightarrow Y$ in a 2-category $\mathbf{K}$, together with a diagram like (41) in the slice $\mathbf{K} / Y$. Suppose that we are able to form the 2-pullback of this diagram along $h$, yielding a diagram

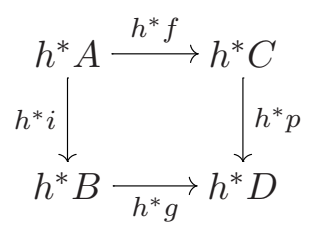

in $\mathbf{K} / X$. Then the pullback of the canonical filler for (41) along $h$ is equal to the canonical filler for (42), where the injective equivalence structure on $h^{*} i$ and the isofibration structure on $h^{*} p$ are those induced by pullback.

Proof. Let us first make clear what the induced structures on $h^{*} i$ and $h^{*} p$ look like. The injective equivalence data for $h^{*} i$ is simply given by applying $h^{*}$ to the corresponding data for $i$. The normal isofibration structure on $h^{*} p$ is given as follows. Let us write $h_{!}: \mathbf{K} / X \rightarrow \mathbf{K} / Y$ for the 2 -functor given by postcomposition with $h$. For any $V \in \mathbf{K} / Y$ whose 2-pullback $h^{*} V$ along $h$ exists, we have 2-natural bijections of categories

$$
\mathbf{K} / Y\left(h_{!} U, V\right) \cong \mathbf{K} / X\left(U, h^{*} V\right) .
$$

In particular, we have bijections between diagrams of the following two forms:
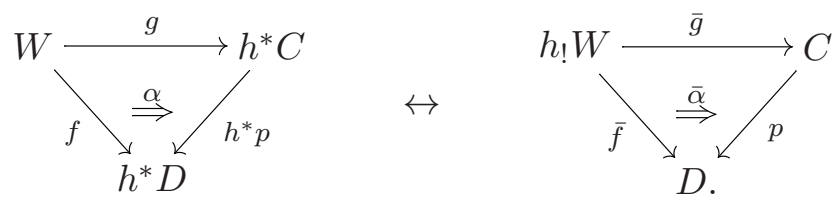

So given an $\alpha$ as on the left of (44), we obtain a lifting for it by first transposing to obtain a 2-cell $\bar{\alpha}$ as on the right of (44). We then apply the isofibration structure of $p$ to obtain $s_{\bar{\alpha}}: h_{!} W \rightarrow C$ and $\sigma_{\bar{\alpha}}: s_{\bar{\alpha}} \Rightarrow \bar{g}$; and finally, we transpose back using (43) to obtain $s_{\alpha}: W \rightarrow h^{*} C$ and $\sigma_{\alpha}: s_{\alpha} \Rightarrow g$. Now, consider the case where $\alpha$ in (44) is itself of the form $h^{*} \beta$ for some $\beta: u \Rightarrow p v: W \rightarrow D$ in $\mathbf{K} / Y$. When this is so, the corresponding $\bar{\alpha}$ is, by naturality, equal to $\beta \circ \epsilon_{W}$, where $\epsilon_{W}: h_{!} h^{*} W \rightarrow W$ is the transpose of $\operatorname{id}_{h^{*} W}$ under the bijection (43). It follows from the definition of isofibration that $s_{\bar{\alpha}}=s_{\beta \circ \epsilon_{W}}=s_{\beta} \circ \epsilon_{W}$ and likewise $\sigma_{\bar{\alpha}}=\sigma_{\beta} \circ \epsilon_{W}$; whereupon transposing under (43) and using naturality, we have $s_{h^{*} \beta}=h^{*}\left(s_{\beta}\right)$ and $\sigma_{h^{*} \beta}=h^{*}\left(\sigma_{\beta}\right)$. Now, according to Proposition 5.1.1, the canonical filler for (42) is given by $s_{\left(h^{*} g\right)\left(h^{*} \theta\right)}=s_{h^{*}(g \theta)}$; and by the above argument this is equal to $h^{*}\left(s_{g \theta}\right)$, which is precisely the pullback along $h$ of the canonical filler for (41), as required.

5.2. Identity types. For the rest of the section, we fix a model of two-dimensional type theory $\mathbb{C}$. We are going to give semantic analogues of each of the logical constructors of $\mathrm{ML}_{2}$ in $\mathbb{C}$. We start with the identity types.

5.2.1. Formation rule. Given $\Gamma \in \mathfrak{C}$ and $A \in \mathfrak{T}(\Gamma)$, we define the semantic identity type on $A$ to be the object $\operatorname{Id}_{A} \in \mathfrak{T}(\Gamma . A . A)$ whose existence is assured by Definition 4.6.1. 
5.2.2. Introduction rule. We recall that the object $\Gamma$.A.A. $\operatorname{ld}_{A} \in \mathfrak{C}$, together with the maps $s:=\pi_{1} \pi_{\mathrm{Id}_{A}}$ and $t:=\pi_{2} \pi_{\mathrm{Id}_{A}}: \Gamma$.A.A.Id $A \rightarrow \Gamma$. $A$, is an arrow object for $\Gamma$. $A$ in $\mathfrak{C} / \Gamma$. As in Proposition 4.1.1, we write $\kappa: s \Rightarrow t$ for the corresponding universal 2-cell. Applying universality of $\kappa$ to the 2-cell

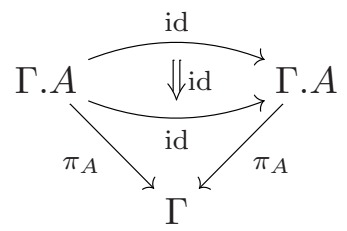

in $\mathfrak{C} / \Gamma$, we obtain a morphism $r_{A}: \Gamma . A \rightarrow \Gamma . A . A . \mathrm{Id}_{A}$ which factorises the diagonal: we have $\pi_{\mathrm{Id}_{A}} r_{A}=\delta_{A}: \Gamma . A \rightarrow \Gamma$.A.A. We call this $r_{A}$ the semantic introduction rule for $\operatorname{ld}_{A}$.

5.2.3. Elimination and computation rules. With reference to Table 1, we require semantic analogues of the premisses $C$ and $d$ of the rule Id-ELIM. These are given by an object $C \in \mathfrak{T}\left(\Gamma \cdot A \cdot A . \mathrm{Id}_{A}\right)$ and a map $d: \Gamma \cdot A \rightarrow \Gamma \cdot A \cdot A . \mathrm{Id}_{A} \cdot C$ of $\mathfrak{C}$ making the following diagram commute:

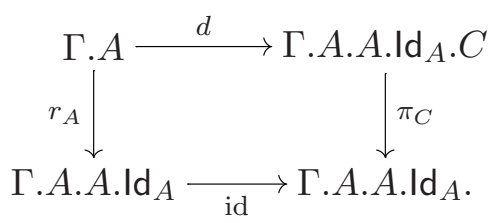

To give a semantic analogue of the conclusion $\mathrm{J}_{d}$, satisfying the analogue of the computation rule, amounts to giving a filler $J_{d}: \Gamma \cdot A \cdot A \cdot \mathrm{Id}_{A} \rightarrow \Gamma \cdot A \cdot A \cdot \mathrm{Id}_{A} \cdot C$ making both sides of (45) commute. Now, by Proposition 3.4.7, $\pi_{C}$ is a normal isofibration in $\mathfrak{C} / \Gamma$; so that if we can show that $r_{A}$ is an injective equivalence in $\mathfrak{C} / \Gamma$, then we may obtain the required filler $J_{d}$ by an application of Proposition 5.1.1. To show that $r_{A}$ is an injective equivalence in $\mathfrak{C} / \Gamma$, we must first give a retraction of $r_{A}$ over $\Gamma$. We take this to be $t: \Gamma$.A.A.ld $A \rightarrow \Gamma$.A (though we could equally well have chosen $s$ ); and we have that $t r_{A}=\mathrm{id}_{\Gamma . A}$ as required. Next we need a 2-cell $\theta: \mathrm{id} \Rightarrow r_{A} t$ over $\Gamma$ satisfying $\theta r_{A}=\mathrm{id}_{r_{A}}$ and $t \theta=\mathrm{id}_{t}$. For this, we consider the following diagram of 1- and 2-cells Г.A.A. $\mathrm{ld}_{A} \rightarrow \Gamma . A:$

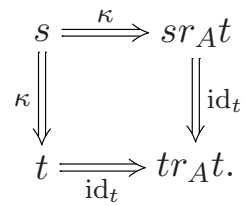

Because $t r_{A}=s r_{A}=\mathrm{id}_{\Gamma . A}$, this diagram is commutative: and so by the two-dimensional aspect of the universal property of $\Gamma$.A.A.ld $A$, is induced by a 2-cell $\theta:$ id $\Rightarrow r_{A} t$ over $\Gamma$ satisfying $s \theta=\kappa$ and $t \theta=\mathrm{id}_{t}$. It remains to verify that $\theta r_{A}=\mathrm{id}_{r_{A}}$. By the uniqueness part of the universal property of $\Gamma$.A.A.Id $A$, it suffices to show that $\kappa \circ \theta r_{A}=\kappa \circ \mathrm{id}_{r_{A}}$. But here we have $\kappa \theta=\kappa\left(r_{A} t\right) \circ s \theta=\mathrm{id}_{t} \circ \kappa=\kappa$ and so $\kappa \circ \theta r_{A}=\kappa r_{A}=\kappa \circ \mathrm{id}_{r_{A}}$ as required.

5.2.4. Stability rules. We now verify that the semantic identity rules given above are stable under semantic substitution. So suppose given $f: \Delta \rightarrow \Gamma$ in $\mathfrak{C}$ together with $A \in$ $\mathfrak{T}(\Gamma)$. We must verify three things. First we must show that reindexing $\Gamma . A . A . \mathrm{Id}_{A}$ along $f$ yields $\Delta . f^{*} A \cdot f^{*} A . \mathrm{ld}_{f^{*} A}$. This follows immediately from the stability requirements of 
Proposition 4.1.2. Next, we must show that the semantic introduction rule $r_{f^{*} A}$ is the reindexing along $f$ of $r_{A}$. This follows from the fact that arrow object structure on $\operatorname{ld}_{f^{*} A}$ is the reindexing of that on $\operatorname{ld}_{A}$ along $f$. Finally, we must show that applications of the semantic elimination rule are stable under substitution. So suppose given a diagram like (45). If we view this as a diagram in $\mathfrak{C} / \Gamma$, then we can reindex it along $f$ to yield a diagram

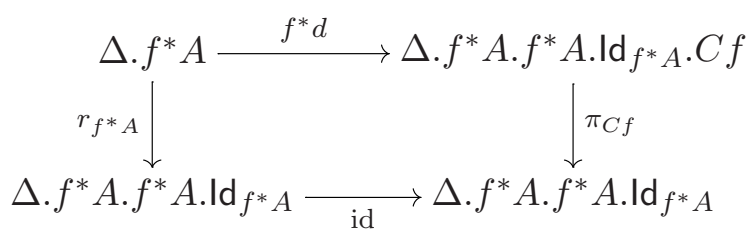

in $\mathfrak{C} / \Delta$. We must show that pulling back the assigned filler for (45) along $f$ yields the assigned filler for (46). Now, by the stability properties of Proposition 3.4.10, we know that the isofibration structure on $\pi_{C f}$ qua map of $\mathfrak{C} / \Delta$ is the one induced by pulling back along $f$ the isofibration structure of $\pi_{C}$ qua map of $\mathfrak{C} / \Gamma$. Moreover, by the stability of the arrow object structure of $\operatorname{Id}_{A}$, the injective equivalence structure on $r_{f^{*} A}$ is the one induced by pulling back that of $r_{A}$ along $f$. The result now follows by applying Proposition 5.1.2.

5.2.5. Remark. Because $\Gamma \cdot A . A . \mathrm{Id}_{A}$ is an arrow object in $\mathfrak{C} / \Gamma \cdot A$, we will in what follows pass back and forward without comment between morphisms $h: \Lambda \rightarrow \Gamma \cdot A \cdot A . \mathrm{ld}_{A}$ and 2-cells $\gamma:$ sh $\Rightarrow t h: \Lambda \rightarrow \Gamma$.A over $\Gamma$.

5.2.6. Discrete identity rules. We now show that the semantic identity rules given above satisfy the semantic equivalents of the rules in Table 2. So suppose given $\Gamma \in \mathfrak{C}$ and $A \in \mathfrak{T}(\Gamma)$ as before. The semantic analogues of the premisses of the rules in Table 2 are a pair of morphisms $a, b: \Gamma \rightarrow \Gamma . A$ of $\mathfrak{C}$ over $\Gamma$, together with a 2-cell

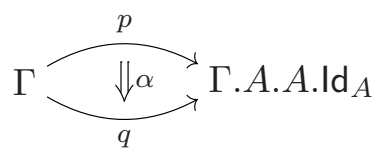

satisfying $s p=s q=a, s \alpha=\mathrm{id}_{a}, t p=t q=b$ and $t \alpha=\mathrm{id}_{b}$. We must show that under these circumstances we have $p=q$ and $\alpha=\mathrm{id}_{p}$. So consider the following diagram of 1and 2-cells $\Gamma \rightarrow \Gamma$.A:

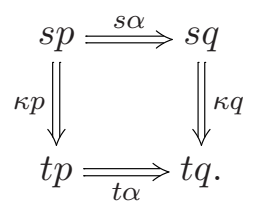

It is commutative, with both sides equal to $\kappa \alpha: s p \Rightarrow t q$; but since $s \alpha=\mathrm{id}_{a}$ and $t \alpha=\mathrm{id}_{b}$, we deduce that $\kappa \alpha=\kappa p=\kappa q: a \Rightarrow b$. By the uniqueness part of the universal property of $\kappa$, this entails that $p=q: \Gamma \rightarrow \Gamma$.A.A.ld $A$. Moreover, we have $\kappa \alpha=\kappa p=\kappa \operatorname{id}_{p}$, and so again by the uniqueness part of the universal property of $\kappa$, we deduce that $\alpha=\mathrm{id}_{p}$ as required.

\subsection{Unit types.}


5.3.1. Formation rule. Given $\Gamma \in \mathfrak{C}$, we define the semantic unit type at $\Gamma$ to be the object $\mathbf{1}_{\Gamma} \in \mathfrak{T}(\Gamma)$ whose existence is assured by Definition 4.6.1.

5.3.2. Introduction rule. Recall that $\mathbf{1}_{\Gamma}$ is a retract bireflection of $\operatorname{id}_{\Gamma}: \Gamma \rightarrow \Gamma$ along the 2-functor $E(\Gamma): \mathfrak{T}(\Gamma) \rightarrow \mathfrak{C} / \Gamma$; so in particular, we have a unit map $u_{\Gamma}: \Gamma \rightarrow \Gamma . \mathbf{1}_{\Gamma}$ over $\Gamma$, and we call this the semantic introduction rule for $\mathbf{1}_{\Gamma}$.

5.3.3. Elimination and computation rules. Suppose given $C \in \mathfrak{T}\left(\Gamma . \mathbf{1}_{\Gamma}\right)$ and a map $d: \Gamma \rightarrow$ $\Gamma . \mathbf{1}_{\Gamma} \cdot C$ of $\mathfrak{C}$ fitting into a commutative diagram

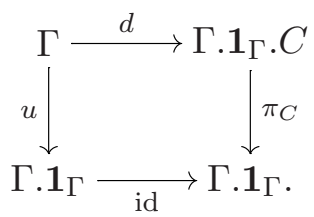

The semantic elimination rule will assign to this data a filler $U: \Gamma . \mathbf{1}_{\Gamma} \rightarrow \Gamma \cdot \mathbf{1}_{\Gamma} \cdot C$ making both triangles commute. Because $\pi_{C}$ is an isofibration in $\mathfrak{C} / \Gamma$, it suffices to show that $u_{\Gamma}$ is a injective equivalence in $\mathfrak{C} / \Gamma$, since then we obtain the desired filler by Proposition 5.1.1. First we must give a retraction for $u_{\Gamma}$ over $\Gamma$. We take this to be $k:=\pi_{\mathbf{1}_{\Gamma}}: \Gamma . \mathbf{1}_{\Gamma} \rightarrow \Gamma$, which satisfies $k u_{\Gamma}=\mathrm{id}_{\Gamma}$ as required. We now give a 2-cell $\theta: \operatorname{id}_{\Gamma .1_{\Gamma}} \Rightarrow u_{\Gamma} k$ satisfying $\theta u_{\Gamma}=\mathrm{id}_{u_{\Gamma}}$ and $k \theta=\mathrm{id}_{k}$. By the two-dimensional aspect of the universal property of $\mathbf{1}_{\Gamma}$, every 2-cell $\alpha: \operatorname{id}_{\Gamma .1_{\Gamma}} \circ u_{\Gamma} \Rightarrow u_{\Gamma} k \circ u_{\Gamma}$ is of the form $\bar{\alpha} \circ u_{\Gamma}$ for a unique 2-cell $\bar{\alpha}: \operatorname{id}_{\Gamma . \mathbf{1}_{\Gamma}} \Rightarrow u_{\Gamma} k$. But because $\operatorname{id}_{\Gamma .1_{\Gamma}} \circ u_{\Gamma}=\operatorname{id}_{u_{\Gamma}}=u_{\Gamma} \circ \operatorname{id}_{\Gamma}=u_{\Gamma} k u_{\Gamma}$, we have in particular the 2-cell $\theta:=\overline{\mathrm{id}_{u_{\Gamma}}}: \operatorname{id}_{\Gamma \cdot 1_{\Gamma}} \Rightarrow u_{\Gamma} k$; which by definition satisfies $\theta u_{\Gamma}=\mathrm{id}_{u_{\Gamma}}$. That it also satisfies $k \theta=\mathrm{id}_{k}$ follows from the fact that $\theta$ is a 2-cell of $\mathfrak{C} / \Gamma$.

5.3.4. Stability rules. We must show that the semantic unit rules are stable under semantic substitution. This follows by an argument entirely analogous to that of $\$ 5.2 .4$, but using the stability properties of Proposition 4.3.2 rather than Proposition 4.1.2.

\subsection{Sum types.}

5.4.1. Formation rule. Given $\Gamma \in \mathfrak{C}, A \in \mathfrak{T}(\Gamma)$ and $B \in \mathfrak{T}(\Gamma . A)$, we define the semantic sum type of $A$ and $B$ to be the object $\Sigma_{A}(B) \in \mathfrak{T}(\Gamma)$ whose existence is assured by Definition 4.6.1.

5.4.2. Introduction rule. $\Sigma_{A}(B)$ is a retract bireflection of $B \in \mathfrak{T}(\Gamma . A)$ along the 2functor $\mathfrak{T}\left(\pi_{A}\right): \mathfrak{T}(\Gamma) \rightarrow \mathfrak{T}(\Gamma \cdot A)$; and so, as in Proposition 4.4.2, we obtain from the unit of this bireflection a map $i: \Gamma \cdot A . B \rightarrow \Gamma . \Sigma_{A}(B)$ of $\mathfrak{C} / \Gamma$. We declare this map to be the semantic introduction rule for $\Sigma_{A}(B)$.

5.4.3. Elimination and computation rules. We consider $C \in \mathfrak{T}\left(\Gamma \cdot \Sigma_{A}(B)\right)$ and a map $d: \Gamma . A . B \rightarrow \Gamma \cdot \Sigma_{A}(B) . C$ of $\mathfrak{C}$ fitting into a commutative diagram

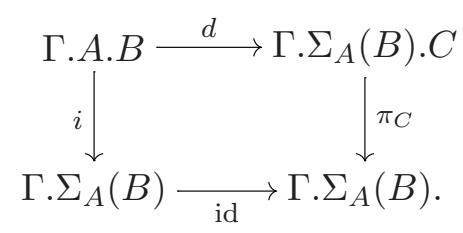

To give the semantic elimination rule satisfying the semantic computation rule is now to give a filler $E: \Gamma \cdot \Sigma_{A}(B) \rightarrow \Gamma \cdot \Sigma_{A}(B) . C$ making both triangles commute. We know 
that $\pi_{C}$ is an isofibration in $\mathfrak{C} / \Gamma$, whilst Definition 4.6.1 assures us that $i$ is an injective equivalence in $\mathfrak{C} / \Gamma$ : thus we obtain the desired filler by applying Proposition 5.1.1.

5.4.4. Stability rules. We must show that the semantic rules for dependent sums are stable under semantic substitution. Again, this follows by an argument analogous to that of $\S 5.2 .4$, this time using the stability properties of Proposition 4.4.3.

5.5. Product types. Finally, we give semantic analogues in $\mathbb{C}$ of the rules for the product types. As in the one-dimensional case, there is a slight mismatch here between the syntax and the semantics. This means that, in addition to the right biadjoints to weakening, we will also need to make use of the semantic unit types of $\S 5.3$. See $[19, \S 5.1-5.3]$ for a fuller discussion of this point.

5.5.1. Formation rule. For $\Gamma \in \mathfrak{C}, A \in \mathfrak{T}(\Gamma)$ and $B \in \mathfrak{T}(\Gamma . A)$, we define the semantic product type of $A$ and $B$ to be the object $\Pi_{A}(B) \in \mathfrak{T}(\Gamma)$ whose existence is assured by Definition 4.6.1.

5.5.2. Application rule. $\Pi_{A}(B)$ is a retract bicoreflection of $B \in \mathfrak{T}(\Gamma . A)$ along $\Delta_{A}:=$ $\mathfrak{T}\left(\pi_{A}\right): \mathfrak{T}(\Gamma) \rightarrow \mathfrak{T}(\Gamma \cdot A)$. The counit of this bicoreflection is a morphism $\epsilon: \Delta_{A} \Pi_{A}(B) \rightarrow$ $B$ of $\mathfrak{T}(\Gamma . A)$. We define the semantic application rule for $\Pi_{A}(B)$ to be the corresponding morphism $\epsilon: \Gamma \cdot A . \Pi_{A}(B) \rightarrow \Gamma . A . B$ of $\mathfrak{C} / \Gamma . A$.

5.5.3. Abstraction and $\beta$-rules. For this, we suppose given, as in the premiss of the abstraction rule, a morphism $f: \Gamma . A \rightarrow \Gamma . A . B$ over $\Gamma$. $A$. We are required to produce from this a map $\lambda(f): \Gamma \rightarrow \Gamma \cdot \Pi_{A}(B)$ over $\Gamma$; which, in order for the $\beta$-rule to hold, should satisfy $\epsilon \circ \Delta_{A}(\lambda(f))=f$. So consider the unit type $\mathbf{1}_{\Gamma \cdot A} \in \mathfrak{T}(\Gamma \cdot A)$. Applying its universal property to $f: \Gamma . A \rightarrow \Gamma . A . B$ yields a morphism $\bar{f}: \Gamma \cdot A . \mathbf{1}_{\Gamma . A} \rightarrow \Gamma . A . B$ over $\Gamma$.A satisfying $\bar{f} \circ u_{\Gamma . A}=f$. We can view $\bar{f}$ as a morphism $\mathbf{1}_{\Gamma . A} \rightarrow B$ of $\mathfrak{T}(\Gamma . A)$; which by the stability of unit types under substitution is equally well a morphism $\bar{f}: \Delta_{A} \mathbf{1}_{\Gamma} \rightarrow B$ of $\mathfrak{T}(\Gamma \cdot A)$. Applying the universal property of $\Pi_{A}(B)$ to this, we obtain a morphism $\overline{\bar{f}}: \mathbf{1}_{\Gamma} \rightarrow \Pi_{A}(B)$ of $\mathfrak{T}(\Gamma)$ satisfying $\epsilon \circ \Delta_{A}(\overline{\bar{f}})=\bar{f}$. This is equally well a morphism $\Gamma . \mathbf{1}_{\Gamma} \rightarrow \Gamma . \Pi_{A}(B)$ over $\Gamma$, so we can now define the map $\lambda(f): \Gamma \rightarrow \Gamma . \Pi_{A}(B)$ over $\Gamma$ to be $\lambda(f):=\overline{\bar{f}} \circ u_{\Gamma}$. It remains to show that we have $\epsilon \circ \Delta_{A}(\lambda(f))=f$; for which we calculate that

$$
\epsilon \circ \Delta_{A}(\lambda(f))=\epsilon \circ\left(\Delta_{A}(\overline{\bar{f}}) \circ \Delta_{A}\left(u_{\Gamma}\right)\right)=\bar{f} \circ u_{\Gamma . A}=f
$$

as required. Here we have used the fact that, by stability of unit types under substitution, we have $\Delta_{A}\left(u_{\Gamma}\right)=u_{\Gamma . A}$.

5.5.4. Function extensionality rules. We now give semantic analogues of the rules of Table 3. For the first rule П-EXT, we suppose given morphisms $m, n: \Gamma \rightarrow \Gamma . \Pi_{A}(B)$ over $\Gamma$, together with a 2-cell

$$
p: \epsilon \circ \Delta_{A}(m) \Rightarrow \epsilon \circ \Delta_{A}(n): \Gamma . A \rightarrow \Gamma . A . B
$$

over $\Gamma$.A. We must produce from this a 2 -cell $\operatorname{ext}(p): m \Rightarrow n$. First we apply the universal property of the unit type $\mathbf{1}_{\Gamma}$ to $m$ and $n$ to obtain morphisms $\bar{m}, \bar{n}: \Gamma \cdot \mathbf{1}_{\Gamma} \rightarrow \Gamma \cdot \Pi_{A}(B)$ over $\Gamma$. These satisfy $m=\bar{m} \circ u_{\Gamma}$ and $n=\bar{n} \circ u_{\Gamma}$, and so we can view $p$ as a 2-cell

$$
p: \epsilon \circ \Delta_{A}(\bar{m}) \circ u_{\Gamma . A} \Rightarrow \epsilon \circ \Delta_{A}(\bar{n}) \circ u_{\Gamma . A}: \Gamma . A \rightarrow \Gamma . A . B,
$$


where again we use stability of unit types under pullback to derive that $\Delta_{A}\left(u_{\Gamma}\right)=u_{\Gamma \cdot A}$. By the two-dimensional aspect of the universal property of $\mathbf{1}_{\Gamma \cdot A}$, we have $p=\bar{p} \circ u_{\Gamma . A}$ for a unique 2-cell

$$
\bar{p}: \epsilon \circ \Delta_{A}(\bar{m}) \Rightarrow \epsilon \circ \Delta_{A}(\bar{n}): \Gamma \cdot A \cdot \mathbf{1}_{\Gamma . A} \rightarrow \Gamma . A . B .
$$

Now, by the two-dimensional aspect of the universal property of $\Pi_{A}(B)$, we have that $\bar{p}=\epsilon \circ \Delta_{A}(\overline{\bar{p}})$ for a unique $\overline{\bar{p}}: \bar{m} \Rightarrow \bar{n}$. We now define the 2 -cell ext $(p)$ to be given by $\overline{\bar{p}} \circ u_{\Gamma}: m \Rightarrow n$.

In order for ext to satisfy the analogue of the rule П-EXT-COMP, we must show that when $m=n$ and $p=\operatorname{id}_{\epsilon \circ \Delta_{A}(m)}$, we have $\operatorname{ext}(p)=\mathrm{id}_{m}$. It suffices for this to show that (with the above notation) $\overline{\bar{p}}=\mathrm{id}_{\bar{m}}: \bar{m} \Rightarrow \bar{m}$; which, by applying successively the universal properties of $\Pi_{A}(B)$ and $\mathbf{1}_{\Gamma . A}$, follows from the fact that $\epsilon \circ \Delta_{A}(\overline{\bar{p}}) \circ u_{\Gamma \cdot A}=p$ is an identity 2-cell. Finally, we must verify that ext satisfies the analogue of the rule П-EXT-APP. Recall from $\S 4.5$ that the operation $*$ appearing in П-EXT-APP is simply the lifting of $\epsilon: \Gamma \cdot A \cdot \Pi_{A}(B) \rightarrow \Gamma \cdot A . B$ to identity types. From this it follows that we must verify that $\epsilon \circ \Delta_{A}(\operatorname{ext}(p))=p: \epsilon \circ \Delta_{A}(m) \Rightarrow \epsilon \circ \Delta_{A}(n)$. We calculate that $\epsilon \circ \Delta_{A}(\operatorname{ext}(p))=$ $\epsilon \circ\left(\Delta_{A}(\overline{\bar{p}}) \circ \Delta_{A}\left(u_{\Gamma}\right)\right)=\bar{p} \circ u_{\Gamma \cdot A}=p$ as required.

5.5.5. Stability rules. We must now show that the semantic rules for dependent products are stable under semantic substitution. This follows by an argument analogous to that of $\S 5.2 .4$; though this time we do not need the stability properties of isofibrations (Proposition 3.4.10) at all; instead, we need those for products (Proposition 4.5.2) and also those for units (Proposition 4.3.2).

5.6. The internal language. We now define the type theory $S(\mathbb{C})$ associated to our two-dimensional model $\mathbb{C}$. It is obtained by recursively extending $\mathrm{ML}_{2}$ with additional inference rules. These inference rules are "axiom" rules with no premisses, and so may be specified by giving only their conclusion. First we have rules introducing new types:

- For each $A \in \mathfrak{T}(1)$ we add a judgement $\vdash \bar{A}$ type.

- For each $A \in \mathfrak{T}(1), B \in \mathfrak{T}(1 . A)$ we add a judgement $x: \bar{A} \vdash \bar{B}(x)$ type.

- And so on.

Then we have rules introducing new terms:

- For each $A \in \mathfrak{T}(1), a \in \in_{1} A$, we add a judgement $\vdash \bar{a}: \bar{A}$.

- For each $A \in \mathfrak{T}(1), B \in \mathfrak{T}(1 . A), b \in \in_{1 . A} B$, we add a judgement $x: \bar{A} \vdash \bar{b}(x)$ : $\bar{B}(x)$.

- And so on.

Here, we use the convention for global sections developed in Notation 3.1.1. Next we have rules identifying the syntactic notions of substitution, weakening, contraction and exchange with their semantic counterparts in $\mathbb{C}$. We give the case of substitution as a representative sample. First we deal with substitution in types:

- For each $A \in \mathfrak{T}(1), B \in \mathfrak{T}(1 . A), a \in \in_{1} 1$. A, we add a judgement $\vdash \bar{B}(\bar{a})=\overline{a^{*} B}$ type.

- For each $A \in \mathfrak{T}(1), B \in \mathfrak{T}(1 . A), C \in \mathfrak{T}(1 . A . B), b \in_{1 . A} B$, we add a judgement $x: \bar{A} \vdash \bar{C}(x, \bar{b}(x))=\overline{b^{*} C}$ type.

- For each $A \in \mathfrak{T}(1), B \in \mathfrak{T}(1 . A), C \in \mathfrak{T}(1 . A . B)$ and $a \in_{1} A$, we add a judgement $y: \bar{B}(\bar{a}) \vdash \bar{C}(\bar{a}, y)=\overline{(a . \iota)^{*} C}$ type.

- And so on.

And now substitution in terms: 
- For each $A \in \mathfrak{T}(1), B \in \mathfrak{T}(1 . A), a \in \in_{1} A, b \in 1 . A B$, we add $\vdash \bar{b}(\bar{a})=\overline{a^{*} b}: \bar{B}(\bar{a})$.

- For each $A \in \mathfrak{T}(1), B \in \mathfrak{T}(1 . A), C \in \mathfrak{T}(1 . A . B), b \in 1 . A B, c \in \in_{1 . A . B} C$, we add $x: \bar{A} \vdash \bar{c}(x, \bar{b}(x))=\overline{b^{*} c}: \bar{C}(x, \bar{c}(x))$.

- For each $A \in \mathfrak{T}(1), B \in \mathfrak{T}(1 . A), C \in \mathfrak{T}(1 . A . B), a \in 1 A, c \in \in_{1 . A . B} C$, we add $y: \bar{B}(\bar{a}) \vdash \bar{c}(\bar{a}, y)=\overline{(a . \iota)^{*} c}: \bar{C}(\bar{a}, y)$.

- And so on.

Finally, we have rules identifying each of the logical rules of $\mathrm{ML}_{2}$ with its semantic counterpart in $\mathbb{C}$. We give only the case of the identity types; the remainder follow the same pattern. First we have the formation rules.

- For each $A \in \mathfrak{T}(1)$, we add $x, y: \bar{A} \vdash \mathrm{Id}_{\bar{A}}(x, y)=\overline{\operatorname{dd}_{A}}(x, y)$ type.

- For each $A \in \mathfrak{T}(1), B \in \mathfrak{T}(1 . A)$, we add

$$
x: \bar{A}, y, z: \bar{B}(x) \vdash \operatorname{ld}_{\bar{B}(x)}(y, z)=\overline{\operatorname{ld}_{B}}(x, y, z) \text { type. }
$$

- And so on.

Next we have the introduction rule. We observe that for $A \in \mathfrak{T}(\Gamma)$, the semantic introduction rule $r_{A}: \Gamma \rightarrow \Gamma$.A.A.ld $A$ over $\Gamma$ can be viewed as a global section $r_{A} \in_{\Gamma} \delta_{A}^{*}\left(\operatorname{ld}_{A}\right)$, where $\delta_{A}: \Gamma . A \rightarrow \Gamma$.A.A is the diagonal morphism. Thus we may add the following rules:

- For each $A \in \mathfrak{T}(1)$, we add $x: \bar{A} \vdash \mathrm{r}(x)=\overline{r_{A}}(x): \operatorname{ld}_{\bar{A}}(x, x)$.

- For each $A \in \mathfrak{T}(1), B \in \mathfrak{T}(1 . A)$, we add

$$
x: \bar{A}, y: \bar{B}(x) \vdash \mathrm{r}(y)=\overline{r_{B}}(x, y): \operatorname{ld}_{\bar{B}(x)}(y, y) .
$$

- And so on.

Finally we come to the identity elimination rule.

- For each $A \in \mathfrak{T}(1), C \in \mathfrak{T}\left(1 . A . A . \operatorname{ld}_{A}\right)$ and $d: 1 . A \rightarrow 1 . A . A . \operatorname{ld}_{A} \cdot C$ as in (45) (which is equally well a global section $d \in_{1 . A} r_{A}^{*} C$ ), we add

$$
x, y: \bar{A}, p: \operatorname{ld}_{\bar{A}}(x, y) \vdash \mathrm{J}_{\bar{d}}(x, y, p)=\overline{J_{d}}(x, y, p): \bar{C}(x, y, p) .
$$

- And so on.

Now, in order for the internal language we have set up to be of any use, we require its types and terms to denote unique elements of the model $\mathbb{C}$. The next Proposition tells us that this is the case.

Proposition 5.6.1 (Soundness). For any $B, C \in \mathfrak{T}\left(1 . A_{1} \cdot A_{2} \ldots A_{n}\right)$, if the judgement

$$
x: \bar{A}_{1} \cdot \bar{A}_{2} \ldots \bar{A}_{n} \vdash \bar{B}(x)=\bar{C}(x) \text { type }
$$

is derivable, then $B=C$. Likewise, for global sections $b, c \in_{1 . A_{1} \ldots A_{n}} B$, if the judgement

$$
x: \bar{A}_{1} \cdot \bar{A}_{2} \ldots \bar{A}_{n} \vdash \bar{b}(x)=\bar{c}(x): \bar{B}(x)
$$

is derivable, then $b=c$.

Proof. By induction on the derivation of the judgement in question, it suffices to show that the semantic counterpart of each syntactic equality rules is satisfied. For the nonlogical equality rules, this is standard (though delicate), and we refer the reader to [15] or [26] for the details (note that we make essential use of the fact that the underlying 1-fibration of $\mathfrak{T} \rightarrow \mathfrak{C}$ is split). The other cases we must consider are the computation rules of Tables 1, 2 or 3, and the rules expressing stability of the logical operations under substitution; and each of these has been dealt with in the preceding sections. 
Remark 5.6.2. Observe that the internal language $\mathrm{S}(\mathbb{C})$ does not give us access to all of the model $\mathbb{C}$ : it only allows us to talk about objects of the base 2-category $\mathfrak{C}$ which have the form $1 . A_{0} \ldots A_{n}$ (where 1 is the given 2 -terminal object). There are two ways around this. We can modify the syntax of our type theory so that contexts and context morphisms are primitive, rather than derived, notions. Then each object or morphism of $\mathfrak{C}$ corresponds directly to a context or context morphism of $\mathrm{S}(\mathbb{C})$. Alternatively, we can keep our type theory the same, and instead work with relative internal languages. Given $\Gamma \in \mathfrak{C}$, the relative internal language $\mathrm{S}_{\Gamma}(\mathbb{C})$ is the type theory whose closed types are objects of $\mathfrak{T}(\Gamma)$, with dependent types being objects of $\mathfrak{T}(\Gamma \cdot A), \mathfrak{T}(\Gamma \cdot A . B)$ and so on. Moreover, because each morphism $\Gamma \rightarrow \Delta$ of $\mathfrak{C}$ induces an interpretation (in the sense of $\S 5.7$ below) $\mathrm{S}_{\Delta}(\mathbb{C}) \rightarrow \mathrm{S}_{\Gamma}(\mathbb{C})$, we obtain what is in an obvious sense a "CC-indexed type theory" 3 .

5.7. Functorial aspects. In Sections 3 and 4, we constructed from each type theory S incorporating $\mathrm{ML}_{2}$ a two-dimensional model $\mathbb{C}(\mathrm{S})$; whilst in the preceding parts of the present Section, we have constructed from each two-dimensional model $\mathbb{C}$ a type theory $\mathrm{S}(\mathbb{C})$ incorporating $\mathrm{ML}_{2}$. It is natural to ask whether these assignations give rise to a functorial semantics in the spirit of [24]. That is, can we define a syntactic category of type theories and a semantic category of models for which the above assignations underlie an equivalence of categories? We finish the paper by sketching a positive answer to this question.

We first define a syntactic category Th. Its objects are the generalised algebraic theories [5] over $\mathrm{ML}_{2}$. These are defined inductively by the following three clauses. Each object of $\mathbf{T h}$ is a sequent calculus; $\mathrm{ML}_{2} \in \mathbf{T h}$; and if $\mathrm{S} \in \mathbf{T h}$, then so is any extension of $\mathrm{S}$. Here, an extension of $\mathrm{S}$ is given by adjoining a set of inference rules each of which has no premisses and a conclusion $\mathcal{J}$ which obeys the following requirements. If $\mathcal{J}$ is of the form $\Gamma \vdash A$ type then $A$ must be fresh for $\mathrm{S}$ and $\Gamma$ must be a well-formed context of $\mathrm{S}$; if $\mathcal{J}$ is of the form $\Gamma \vdash a: A$ then $a$ must be fresh for $\mathrm{S}$ and $\Gamma \vdash A$ type must be derivable in $\mathrm{S}$; if $\mathcal{J}$ is of the form $\Gamma \vdash A=B$ type then $\Gamma \vdash A$ type and $\Gamma \vdash B$ type must be derivable in $\mathrm{S}$; and finally if $\mathcal{J}$ is of the form $\Gamma \vdash a=b: A$ then $\Gamma \vdash a: A$ and $\Gamma \vdash b: A$ must be derivable in $\mathrm{S}$. Note that the assignation $\mathbb{C} \mapsto \mathrm{S}(\mathbb{C})$ sends each two-dimensional model to a GAT over $\mathrm{ML}_{2}$.

The morphisms of $\mathbf{T h}$ are equivalence classes of interpretations. Given $\mathrm{S}, \mathrm{T} \in \mathbf{T h}$, an interpretation $F: \mathrm{S} \rightarrow \mathrm{T}$ is a function $F$ taking derivable judgements of $\mathrm{S}$ to derivable judgements of $\mathrm{T}$, subject to the following requirements. Each $F(A$ type) should have the form $F A$ type; each $F(a: A)$ should have the form $F a: F A$; each $F(A=B$ type) should have the form $F A=F B$ type; and each $F(a=b: A)$ should have the form $F a=F b: F A$. Moreover, if we suppose $F(\Gamma \vdash A$ type) has the form $F \Gamma \vdash F A$ type, then each $F(\Gamma, x: A \vdash B(x)$ type) should have the form $F \Gamma, x: F A \vdash F B(x)$ type; each $F(\Gamma, x: A \vdash b(x): B(x))$ should have the form $F \Gamma, x: F A \vdash F b(x): F B(x)$; and similarly for the two equality judgement forms. Finally we require that $F$ should commute with all the inference rules of $\mathrm{ML}_{2}$. We give the case of the rule of Id-formation for illustration. Suppose given a derivable judgement $\Gamma \vdash A$ type in S. We write its image under $F$ as $F \Gamma \vdash F A$ type, and the image of $\Gamma, x, y: A \vdash \operatorname{ld}_{A}(x, y)$ type as

\footnotetext{
${ }^{3} \mathrm{~A}$ finer analysis shows that this is really a two-dimensional indexing. That is, we have a trihomomorphism $\mathfrak{C}^{\text {coop }} \rightarrow \mathbf{T h}$, where $\mathbf{T h}$ is a suitably-defined tricategory of two-dimensional theories.
} 
$F \Gamma, x, y: F A \vdash F \operatorname{ld}_{A}(x, y)$ type. Now the following judgement should be derivable in $\mathrm{T}$ :

$$
F \Gamma, x, y: F A \vdash \operatorname{Id}_{F A}(x, y)=F \operatorname{ld}_{A}(x, y) \text { type. }
$$

The equivalence relation we impose on interpretations identifies $F, G: \mathrm{S} \rightarrow \mathrm{T}$ if they differ only up to definitional equality in the obvious sense. It is now straightforward to show that GATs and equivalence classes of interpretations form a category $\mathbf{T h}$.

Remark 5.7.1. Using the above notion of interpretation, we can now say what it means to give an interpretation of a GAT $\mathrm{T}$ in a two-dimensional model $\mathbb{C}$ : namely, to give an interpretation (in the above sense) $\mathrm{T} \rightarrow \mathrm{S}(\mathbb{C})$. It is easy to check that this accords with the intuitive syntactic notion we would give.

We now define a semantic category Mod. Its objects are models of two-dimensional type theory as in Definition 4.6.1. A morphism $F: \mathbb{C} \rightarrow \mathbb{C}^{\prime}$ is given by a pair of 2-functors $F_{1}: \mathfrak{C} \rightarrow \mathfrak{C}^{\prime}$ and $F_{2}: \mathfrak{T} \rightarrow \mathfrak{T}^{\prime}$ rendering commutative the squares

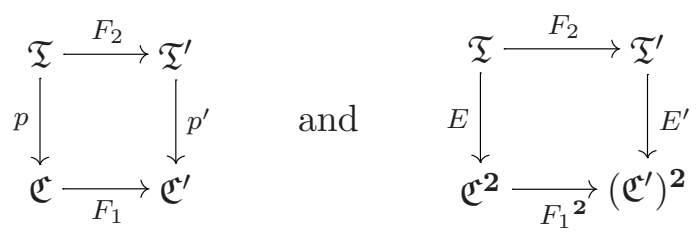

and preserving all the additional structure on the nose.

Proposition 5.7.2. The assignations $\mathrm{S} \mapsto \mathbb{C}(\mathrm{S})$ and $\mathbb{C} \mapsto \mathrm{S}(\mathbb{C})$ underlie functors $\mathbb{C}(-):$ Th $\rightarrow$ Mod and $\mathrm{S}(-):$ Mod $\rightarrow$ Th.

Proof. Given an interpretation $F: \mathrm{S} \rightarrow \mathrm{T}$, we define functors $G_{0}: \mathfrak{C}(\mathrm{S}) \rightarrow \mathfrak{C}(\mathrm{T})$ and $G_{1}: \mathfrak{T}(\mathrm{S}) \rightarrow \mathfrak{T}(\mathrm{T})$ by an obvious structural induction over the objects and morphisms of the domain categories. In order to extend these functors to 2 -functors, we first show by induction that every object $\Gamma \in \mathfrak{C}(\mathrm{S})$ has an accompanying arrow object given by the identity context $\mathrm{Id}_{\Gamma}$. But now, since $F$ preserves the identity type structure, the corresponding $G_{0}$ will preserve these arrow objects; hence we may extend $G_{0}$ to a 2 -functor by regarding each 2-cell of $\mathfrak{C}(\mathrm{S})$ as a 1-cell into an arrow object, mapping this 1-cell over and then turning the resulting 1-cell back into a 2-cell of $\mathfrak{C}(\mathrm{T})$. Because the comprehension 2functors of $\mathbb{C}(\mathrm{S})$ and $\mathbb{C}(\mathrm{T})$ are 2-fully faithful, this in turn determines the extension of $G_{1}$ to a 2 -functor. Finally, the fact that $F$ strictly preserves the remaining structure implies that the same is true of $\left(G_{0}, G_{1}\right)$, and so we obtain a morphism of models $\mathbb{C}(\mathrm{S}) \rightarrow \mathbb{C}(\mathrm{T})$ as required.

Conversely, given a morphism of models $F: \mathbb{C} \rightarrow \mathbb{C}^{\prime}$, we may define an interpretation $\mathrm{S}(\mathbb{C}) \rightarrow \mathrm{S}\left(\mathbb{C}^{\prime}\right)$ as follows. By structural induction, every closed type $A$ of $\mathrm{S}(\mathbb{C})$ is definitionally equal to one of the form $\bar{X}$ for some $X \in \mathfrak{T}(1)$; and by Proposition 5.6.1, this $X$ is unique. Thus we may define the image of $A$ under the interpretation to be the type $\overline{G_{1}(X)}$ of $\mathrm{S}\left(\mathbb{C}^{\prime}\right)$. Similarly, every closed term $a: A$ of $\mathrm{S}(\mathbb{C})$ is definitionally equal to one of the form $\bar{x}: \bar{X}$ for a unique map $x: 1 \rightarrow 1 . X$ of $\mathfrak{C}$, and so we may define $F(a)$ to be the term $\overline{G_{0}(x)}: \overline{G_{1}(X)}$. This definition extends to types and terms in non-empty contexts in an obvious way. Finally, the fact that our morphism of models preserves all the remaining structure on the nose implies the same for the interpretation just described. 
However, the functors defined in this Proposition do not give rise to an equivalence of categories. There are two reasons for this. The first is the issue raised in Remark 5.6.2. Observe that any two-dimensional model in the image of $\mathbb{C}(-)$ has the property that each object $\Gamma \in \mathfrak{C}$ is of the form $1 . A_{1} \ldots A_{n}$ for a unique (possibly empty) sequence of objects $A_{1} \in \mathfrak{T}(1), \ldots, A_{n} \in \mathfrak{T}\left(1 . A_{1} \ldots A_{n-1}\right)$. This is the "tree condition" of [5]. Clearly not every two-dimensional model has this property, so that if we are to obtain an equivalence, we must first cut down to the full sub-2-category $\mathbf{M o d}_{t r} \subset$ Mod on those which do. The second reason we do not obtain an equivalence is more subtle. In order for $\mathbf{T h} \simeq \mathbf{M o d}_{t r}$ to hold, we must certainly have for each $S \in \mathbf{T h}$ that $\mathrm{S}(\mathbb{C}(\mathrm{S})) \cong \mathrm{S}$. However, this turns out not to be the case: we run into problems with the terms witnessing the elimination rules. As an illustration, we will show that $\mathrm{ML}_{2} \nsucceq \mathrm{S}\left(\mathbb{C}\left(\mathrm{ML}_{2}\right)\right)$. Because the object $\mathrm{ML}_{2}$ is initial in $\mathbf{T h}$, there is a unique morphism $F: \mathrm{ML}_{2} \rightarrow \mathrm{S}\left(\mathbb{C}\left(\mathrm{ML}_{2}\right)\right)$ : and so it suffices to show that $F$ is not surjective. First observe that by 1-elimination we can derive a judgement

$$
z: \mathbf{1} \vdash \mathrm{U}_{\star}(z): \mathbf{1}
$$

in $\mathrm{ML}_{2}$. Next note that the judgements of $\mathrm{S}\left(\mathbb{C}\left(\mathrm{ML}_{2}\right)\right)$ are simply equivalence classes of judgements of $\mathrm{ML}_{2}$ with respect to definitional equality; and so by passing to the quotient, we obtain from (47) a judgement

$$
z:[\mathbf{1}] \vdash\left[\mathrm{U}_{\star}\right](z):[\mathbf{1}]
$$

of $\mathrm{S}\left(\mathbb{C}\left(\mathrm{ML}_{2}\right)\right)$. The crucial point is that (48) does not coincide with the value of $F$ at the judgement (47). This latter can be described as follows. First we derive a term $z: 1 \vdash \phi(z): \operatorname{ld}_{1}(z, \star)$ in $\mathrm{ML}_{2}$ by 1-elimination, taking $\phi(z):=\mathrm{U}_{\mathrm{r}(\star)}(z)$. Now by the description of the semantic unit types given in $\S 5.3$, we see that applying $F$ to (47) yields (up to definitional equality) the following judgement in $\mathrm{S}\left(\mathbb{C}\left(\mathrm{ML}_{2}\right)\right.$ ):

$$
z:[\mathbf{1}] \vdash\left[\phi(z)^{*}(\star)\right]:[\mathbf{1}] .
$$

Now, if (49) were definitionally equal to (48), then we would also have that $z: \mathbf{1} \vdash$ $\mathrm{U}_{\star}(z)=\phi(z)^{*}(\star): \mathbf{1}$ in $\mathrm{ML}_{2}$, and this is not the case. Hence $F$ applied to (47) does not yield (48), from which it follows by induction over derivable judgements of $\mathrm{ML}_{2}$ that (48) cannot lie in the image of $F: \mathrm{ML}_{2} \rightarrow \mathrm{S}\left(\mathbb{C}\left(\mathrm{ML}_{2}\right)\right.$ ).

There are several ways in which we can resolve this issue. The first is for us to change our notion of model so that it accords more closely with the type theory. This is unsatisfactory, as we have then reverted to a categorical paraphrasing of type theoretic syntax. A second alternative is to change our notion of type theory so that it accords more closely with the categorical model. This involves removing the elimination rules altogether, and instead taking the Leibniz rule, together with the injective equivalence structures on the introduction terms, as primitives. This is unsatisfactory for a more subtle reason. Whilst it may be reasonably straightforward to give this alternative presentation for two-dimensional type theory, we would find as we moved towards full intensional type theory that it would require a more and more intricate set of rules expressing appropriate coherence properties of our new primitives. The elegant simplicity of intensional type theory would be lost completely.

A third solution, and our preferred one, is to equip our categories of theories and of models with more generous notions of morphism, ones which preserve some of the structure only up to propositional, rather than definitional equality. There is a great 
deal of scope in how far we go with this. In the present paper, we make only the minimal modifications necessary to obtain the desired equivalence. A fuller treatment would take account of the fact that our models and theories are themselves two-dimensional structures, so that their respective totalities should give rise not merely to equivalent categories, but also to triequivalent Gray-categories (=semi-strict 3-categories) in the sense of [11]. Adopting this more comprehensive approach would be necessary if, for instance, we wished to study the 2-category of interpretations of some generalised algebraic theory inside a particular two-dimensional model. However, for our present purposes, we do not need to go this far; and so, in the interests of brevity, we do not.

The minimal modification that we will consider is given as follows. On the syntactic side, we define a category $\mathbf{T h}_{\psi}$ with as objects GATs over $\mathrm{ML}_{2}$ and as maps $F: \mathrm{S} \rightarrow \mathrm{T}$ pseudo-interpretations, whose definition generalises that of an interpretation by dropping the requirement that $F$ should preserve each of the following rules up to definitional equality: 1-ELIM, Id-ELIM, $\Sigma$-ELIM, and $\Pi$-ABS. One may now think that, in order to justify the name pseudo-interpretation, we should ask for $F$ to preserve these rules at least up to propositional equality; but it turns out that this is unnecessary, because this weaker form of preservation is a consequence of the type-theoretic elimination rules.

On the semantic side we define a category $\mathbf{M o d}_{\psi}$ whose objects are two-dimensional models, and whose maps $F: \mathbb{C} \rightarrow \mathbb{C}^{\prime}$ are pseudo-morphisms. These are obtained by relaxing in the definition of morphism of models the requirement that the following structure should be preserved: the normal isofibration structures on dependent projections $\pi_{A}$; the injective equivalence structures on the maps $i: \Gamma . A . B \rightarrow \Gamma \cdot \Sigma_{A}(B)$ associated to dependent sums; and the assignations $f \mapsto \bar{f}$ on 1-cells associated to the unit types, dependent sums, and dependent products. Once again, we do not need to add conditions requiring these pieces of structure to be preserved up to isomorphism, since this will be an automatic consequence of the remaining structure. As before, we write $\left(\mathbf{M o d}_{\psi}\right)_{t r}$ for the full subcategory of $\mathbf{M o d}_{\psi}$ on those models satisfying the tree condition.

Proposition 5.7.3. The functors $\mathbb{C}(-)$ and $\mathrm{S}(-)$ extend to functors $\mathbf{T} \mathbf{h}_{\psi} \rightarrow(\mathbf{M o d})_{t r}$ and $\left(\mathbf{M o d}_{\psi}\right)_{t r} \rightarrow \mathbf{T} \mathbf{h}_{\psi}$ respectively.

Proof. The argument of Proposition 5.7.2 carries over almost entirely unmodified. The only subtlety arises in defining the pseudo-morphism of models $\mathbb{C}(\mathrm{S}) \rightarrow \mathbb{C}(\mathrm{T})$ corresponding to a pseudo-interpretation $F: \mathrm{S} \rightarrow \mathrm{T}$. As before, we define functors $G_{0}$ and $G_{1}$ by induction on the objects and morphisms of $\mathbb{C}(\mathrm{S})$; but when it comes to extending these to 2 -functors, we encounter the problem that the interpretation $F$, since it no longer preserves the rule Id-ELIM, may not send identity contexts to identity contexts. However, using the fact that Id-ELIM is preserved at least up to propositional equality, we may show by induction that $F$ will send an identity context to something isomorphic to an identity context. From this, it follows that $G_{0}$ will still preserve arrow objects, so that we may continue the argument as before.

But now we have that:

Proposition 5.7.4. The functors $\mathbb{C}(-)$ and $\mathrm{S}(-)$ induce an equivalence of categories $\left(\operatorname{Mod}_{\psi}\right)_{t r} \simeq \mathbf{T h}_{\psi}$.

Proof. First observe that if $\mathbb{C}$ is a model satisfying the tree condition, then the contexts and context morphisms of $\mathrm{S}(\mathbb{C})$ are, up to definitional equality, just the objects and morphisms of $\mathfrak{C}$, whilst the types-in-context of $\mathrm{S}(\mathbb{C})$ are just the objects of $\mathfrak{T}$. From this it 
follows that $\mathrm{S}(-)$ is fully faithful. Indeed, given a pseudo-interpretation $F: \mathrm{S}(\mathbb{C}) \rightarrow \mathrm{S}\left(\mathbb{C}^{\prime}\right)$, our observation allows us to define functors $G_{0}: \mathfrak{C} \rightarrow \mathfrak{C}^{\prime}$ and $G_{1}: \mathfrak{T} \rightarrow \mathfrak{T}^{\prime}$. As in the last proof, the pseudo-interpretation $F$ must send an identity context to something isomorphic to an identity context; from which it follows that $G_{0}$ preserves arrow objects, allowing us to extend $G_{0}$ and $G_{1}$ to 2 -functors as before. It is now easy to verify that the resultant pair $\left(G_{0}, G_{1}\right)$ is a pseudo-morphism; and by examining the proof of Proposition 5.7.2, we see that it is sent by $\mathrm{S}(-)$ to $F$ and that it is the unique pseudo-morphism with this property.

It remains to show that for each $\mathrm{S} \in \mathbf{T h}_{\psi}$ we have an isomorphism $\mathrm{S} \cong \mathrm{S}(\mathbb{C}(\mathrm{S}))$ in $\mathbf{T h}_{\psi}$, and that these are natural in S. Now, up to definitional equality, the judgements of $\mathrm{S}(\mathbb{C}(\mathrm{S}))$ are the same as those of $\mathrm{S}$; and so we obtain mutually inverse assignations between the judgements of the former and those of the latter. Moreover, by following through the constructions of $\mathbb{C}(-)$ and $S(-)$, we see that all of the logical structure of $\mathrm{S}(\mathbb{C}(\mathrm{S}))$ is given as in $\mathrm{S}$, with the possible exception of the rules 1-ELIM, Id-ELIM, $\Sigma$-ELIM, and $\Pi$-ABS (as seen in the discussion following Proposition 5.7.2). But this says precisely that these mutually inverse assignations are pseudo-interpretations, and so give rise to a natural isomorphism $\mathrm{S} \cong \mathrm{S}(\mathbb{C}(\mathrm{S}))$ in $\mathbf{T h}_{\psi}$ as required.

\section{REFERENCES}

[1] Aczel, P. Notes towards a formalisation of constructive Galois theory, 1994. Manuscript, University of Manchester. 2

[2] Awodey, S., And Warren, M. Homotopy theoretic models of identity types. Mathematical Proceedings of the Cambridge Philosophical Society 146, 1 (2009), 45-55. 1, 2, 21, 33

[3] Bousfield, A. Constructions of factorization systems in categories. Journal of Pure and Applied Algebra 9, 2-3 (1977), 207-220. 21

[4] Bruijn, N. D. Telescopic mappings in typed lambda calculus. Information and Computation 91, 2 (1991), 189-204. 4

[5] Cartmell, J. Generalised algebraic theories and contextual categories. Annals of Pure and Applied Logic 32 (1986), 209-243. 8, 41, 43

[6] Dybjer, P. Internal type theory. In Types for proofs and programs (Torino, 1995), vol. 1158 of Lecture Notes in Computer Science. Springer, 1996, pp. 120-134. 1, 8

[7] Ehrhard, T. Une sémantique catégorique des type dépendents. Application au Calcul des Constructions. PhD thesis, Université Paris VII, 1988. 8

[8] Gambino, N. Homotopy limits for 2-categories. Mathematical Proceedings of the Cambridge Philosophical Society 145, 1 (2008), 43-63. 21

[9] Gambino, N., And Garner, R. The identity type weak factorisation system. Theoretical Computer Science 409 (2008), 94-109. 1, 21

[10] Garner, R. On the strength of dependent products in the type theory of Martin-Löf. Annals of Pure and Applied Logic 160 (2009), 1-12. 1

[11] Gordon, R., Power, J., And Street, R. Coherence for tricategories. Memoirs of the American Mathematical Society 117, 558 (1995). 16, 44

[12] Grandis, M., And Tholen, W. Natural weak factorization systems. Archivum Mathematicum 42, 4 (2006), 397-408. 33

[13] Hermida, C. Some properties of Fib as a fibred 2-category. Journal of Pure and Applied Algebra 134, 1 (1999), 83-109. 16

[14] Hofmann, M. Elimination of extensionality in martin-löf type theory. In Types for proofs and programs (Nijmegen, 1993), vol. 806 of Lecture Notes in Computer Science. Springer, 1994, pp. 166190. 2

[15] Hofmann, M. Extensional concepts in intensional type theory. PhD thesis, University of Edinburgh, 1995. 1,40 
[16] Hofmann, M. On the interpretation of type theory in locally cartesian closed categories. In Computer science logic (Kazimierz, 1994), vol. 933 of Lecture Notes in Computer Science. Springer, 1995, pp. 427-441. 1, 2

[17] Hofmann, M., And Streicher, T. The groupoid interpretation of type theory. In Twenty-five years of constructive type theory (Venice, 1995), vol. 36 of Oxford Logic Guides. Oxford University Press, 1998, pp. 83-111. 1, 2, 11

[18] Hyland, J. M., And Pitts, A. The theory of constructions: categorical semantics and topostheoretic models. In Categories in computer science and logic (Boulder, CO, 1987), vol. 92 of Contemporary Mathematics. American Mathematical Society, 1989, pp. 137-199. 8

[19] JacoBs, B. Comprehension categories and the semantics of type dependency. Theoretical Computer Science 10\%, 2 (1993), 169-207. 8, 9, 38

[20] JACoBs, B. Categorical logic and type theory, vol. 141 of Studies in Logic and the Foundations of Mathematics. North-Holland Publishing Company, 1999. 4

[21] Kelly, G. M. Basic concepts of enriched category theory, vol. 64 of London Mathematical Society Lecture Note Series. Cambridge University Press, 1982. 26

[22] Kelly, G. M. Elementary observations on 2-categorical limits. Bulletin of the Australian Mathematical Society 39, 2 (1989), 301-317. 26

[23] Kelly, G. M., And Street, R. Review of the elements of 2-categories. In Category Seminar (Proc. Sem., Sydney, 1972/1973), vol. 420 of Lecture Notes in Mathematics. Springer, 1974, pp. 75-103. 8

[24] Lawvere, F. W. Some algebraic problems in the context of functorial semantics of algebraic theories. In Reports of the Midwest Category Seminar II, vol. 61 of Lecture Notes in Mathematics. Springer, 1968, pp. 41-61. 3, 41

[25] Nordström, B., Petersson, K., and Smith, J. Programming in Martin-Löf's Type Theory, vol. 7 of International Series of Monographs on Computer Science. Oxford University Press, 1990. 1, 3, 6

[26] PitTs, A. M. Categorical logic. In Handbook of Logic in Computer Science, Volume 5. Algebraic and Logical Structures, S. Abramsky, D. M. Gabbay, and T. S. E. Maibaum, Eds. Oxford University Press, 2000, pp. 39-128. 40

[27] Seely, R. Locally cartesian closed categories and type theory. Mathematical Proceedings of the Cambridge Philosophical Society 95, 1 (1984), 33-48. 1

[28] Street, R. Fibrations and Yoneda's lemma in a 2-category. In Category Seminar (Proc. Sem., Sydney, 1972/1973), vol. 420 of Lecture Notes in Mathematics. Springer, 1974, pp. 104-133. 28

[29] TAYlor, P. Practical foundations of mathematics, vol. 59 of Cambridge Studies in Advanced Mathematics. Cambridge University Press, 1999. 8

Department of Pure Mathematics and Mathematical Statistics, Wilberforce Road, CamBRIDGE CB3 0WB, UK

E-mail address: rhgg2@cam.ac.uk 